\title{
Perturbazioni geomagnetiche, aurorali, ionosferiche e dei raggi cosmici: interdipendenze e relazioni con l'attività solare
}

\author{
F. Mariani $(*)-$ F. MLOLINa $(* *)$
}

Ricevuto il 6 Dicembre 1962

\begin{abstract}
Riassuxto. - In questa prima parte del Capitolo $V$ della presente rassegna viene esaminata la situazione fisica dello spazio esterno alla Terra. specialmente per quanto riguarda il campo magnetico e le particelle in esso "intrappolate".

Per la distribuzione del campo magnetico, vengono riportate innanzi tutto le deduzioni ottenute indirettamente dalleffetto di latitudine della intensita della radiazione cosmica, con particolare riguardo al cosidetto "equatore dei raggi cosmici ", e vengono poi illustrati i primi risultati delle misure dirette dei campi magnetici nella esosfera eseguite a mezzo dei satelliti e delle sonde spaziali, risultati che, complessivamente, fanno pensare che il confine fra il campo geomagnetico e il campo magnetico interplanetario sia a una distanza di $70.000-80.000 \mathrm{~km}$ dalla Terra.

Segue una breve descrizione delle informazioni che si possono ottenere a mezzo dei whistlers sulla densità elettronica a distanza della Terra dell ordine di alcune migliaia di chilometri.

Vengono poi illustrate le caratteristiche delle fasce di radiazione di Van Allen (morfologia, intensiti, natura e spettro energetico delle particelle che le costituiscono), nonché delle loro variazioni temporali soprattutto in dipendenza delle perturbazioni magnetiche, e vengono brevemente passate in rassegna alcune delle ipotesi avanzate sulla origine delle fasce.

Vengono infine illustrati, fra gri effetti geofisici delle esplosioni nucleari in alta quota, quelli che hanno speciale attinenza con gli argomenti trattati in questa parte, e precisamente quelli che possono fornire informazioni o indieazioni sull andamento delle linee di forza del campo magnetico terrestre e sull'intrappolanento delle particelle.
\end{abstract}

(*) Istituto di Nisica dell'Lniversita e Commissione Italiana per le Ricerche Spaziali del C.N.R., Roma: Istituto Nazionale di Geofisiea, Roma.

(**) Istituto Nazionale di Geofisica, Roma. 
Scmmary. - In the present review, the first part of Chapter $V$ is roncerned with an examination of the physical situation in space surroundjug the earth. especially as regards the magnetic field and the "trapped" particles.

For the distribution of the magnetic field, the report first of all gives all the deductions obtained indireetly from the latiturle effect of the intensity of the cosmic radiation, with particular regard to the so-called "cosmic ray equator" "; there follow the first results of the direct measurements of the mannetic fields in the exosphere, carried out by means of satellites and space probes. These results, consiclered as a whole, suggest that the boundary between the geomagnetic field and the interplanetary magnetic field may lie at a distance of $70,000-80,000 \mathrm{Km}$. from the Earth.

There follows a brief description of the information obtainable by means of whistlers, concerning the electron density at a distance from the Liarth of the order of several thousand kilometers.

The characteristies of the Van Allen radiation belts are then illustrated (morphology, intensity, nature and energy spectrum of the particles of which they are formed), as well as their time variations, expecially as rearards their dependence upon magnetic disturbances, and a brief mention is made of the theories advanced regarding the origrin of these belts.

In conclusion, with regard to the geophysical effects of high-altitude nuclear explosions, the report deseribes those that have a special connection with the present subject: namely, those that can provide information or indications concerning the behaviour of the lines of force of the earth magnetic field and the trapping of the particles.

\title{
5. STATO FISICO DELLO SPAZIO INTERPLANETARIO (*)
}

\author{
a. - CAMPO MAGNETICO NELL ESOSFERA \\ E (AMPO INTERPJANETARIO. FASCE DI RADIAZIONE
}

La conoscenza delle condizioni fisiche a quote superiori al centinaio di km dal suolo o assai interessante perché in questa regione ha inizio il passaggio più o meno graduale dalla atmosfera alla situazione fisica propria dello spazio interplanetario, e perché solo in questi ultimi anni si è potuto cominciare a eflettuare rilevazioni " in loco ".

Ci limitiamo nel seguito a considerare solo quelli dei risultati sperimentali che in qualche modo interessano la situazione magnetica, elettromagnetica e colpuscolare nello spazio prossimo alla Terra.

(*) I primi quattro eapitoli di questo lavoro sono pubblicati in "An11. Geof." 12, 297 e 389 (1959); 13, 85 e 135 (1960). 


\subsection{Il campo magnetico nello spazio intorno alla Terra.}

La conoseenza della distribuzione del ampo magnetico terrestre intorno alla Terra, a distanze dellordine del raggio terrestre, a la sua "Saldatura » con quello eventuale del Sole o, comunque, con quello esistente nello spazio interplanetario ha grande importanza nello studio delle perturbazioni geomagnetiche, auromali, ionosferiche e dei raggi cosmici rhe abbiano considerato nei precedenti appitoli. In effetti, la distribuzione del campo magnetico nello spazio interplanetario, regolare o no nello spazio, costante o no nel tempo, condiziona in modo essenziale il moto e la distribuzione nello spazio delle particelle cariche eui molta parte dei fatti sperimentali induce ad attribuire la causal delle perturbazioni stesse.

Particolare importanza laa, a tale riguardo, lo studio della distribuzione dell'intensità della ladiazione cosmical primaria, la cui densità nello spazio de talnonte bassa da poter ritenere le particelle che la costituiscono esenti da interazioni di sorta; le singole particelle risentono cosi del solo efletto del campo magnetico nello spazio, in particolare di quello interplanetario e terrestre, sui quali si possono cosi ottenere importanti informazioni.

5.1.1. Posizione dell'equatore geomagnetico e radiazione cosmica. - La analisi in serie di funzioni armoniche sferiche del eampo geomagnetico superficiale intrapresa da Gauss, continuata e affinata successivamente da numerosi Autori, consente di definire con noterole precisione la distribuzione del campo geomagnetico intorno alla Terra; nella approssimazione di dipolo ne vengono cosi definiti il momento a l'orientanento. Resta in particolare definita come equatore geomagnetico la linea intersezione del piano equatoriale del dipolo con la superficie terrestre. Sella detta apperssimazione, il dipolo ha il suo centro nel centro della Terra e i suoi poli magnetici nord a sud puntano rispettivamente verso i punti di coordinate geografiche $\left(7805 \mathrm{~N}, 291^{\circ} \mathrm{F}\right)$ a $\left(7805 \mathrm{~S}, 111^{\circ} \mathrm{E}\right)$ rispettivamente.

Aumentando il numero di termini presi in considerazione si oftengono espressioni meglio approssimate del campo magnetico terrestre. In particolare, la fig. 5.1 mostra (1) l"intersezione delle linee di forza del campo magnetico con la superficie della Terra per l'emisfero nord e sud, calcolate con l'uso dei primi 48 coefficienti di Gauss. 
I'interpretazione degli effetti geomagnetici sulla radiazione cosmica (v. 4.2) i stata per molto tempo fatta con riferimento al campo magnetico prodotto da un dipolo centrato; tuttavia, negli ultimi anni, il raf-

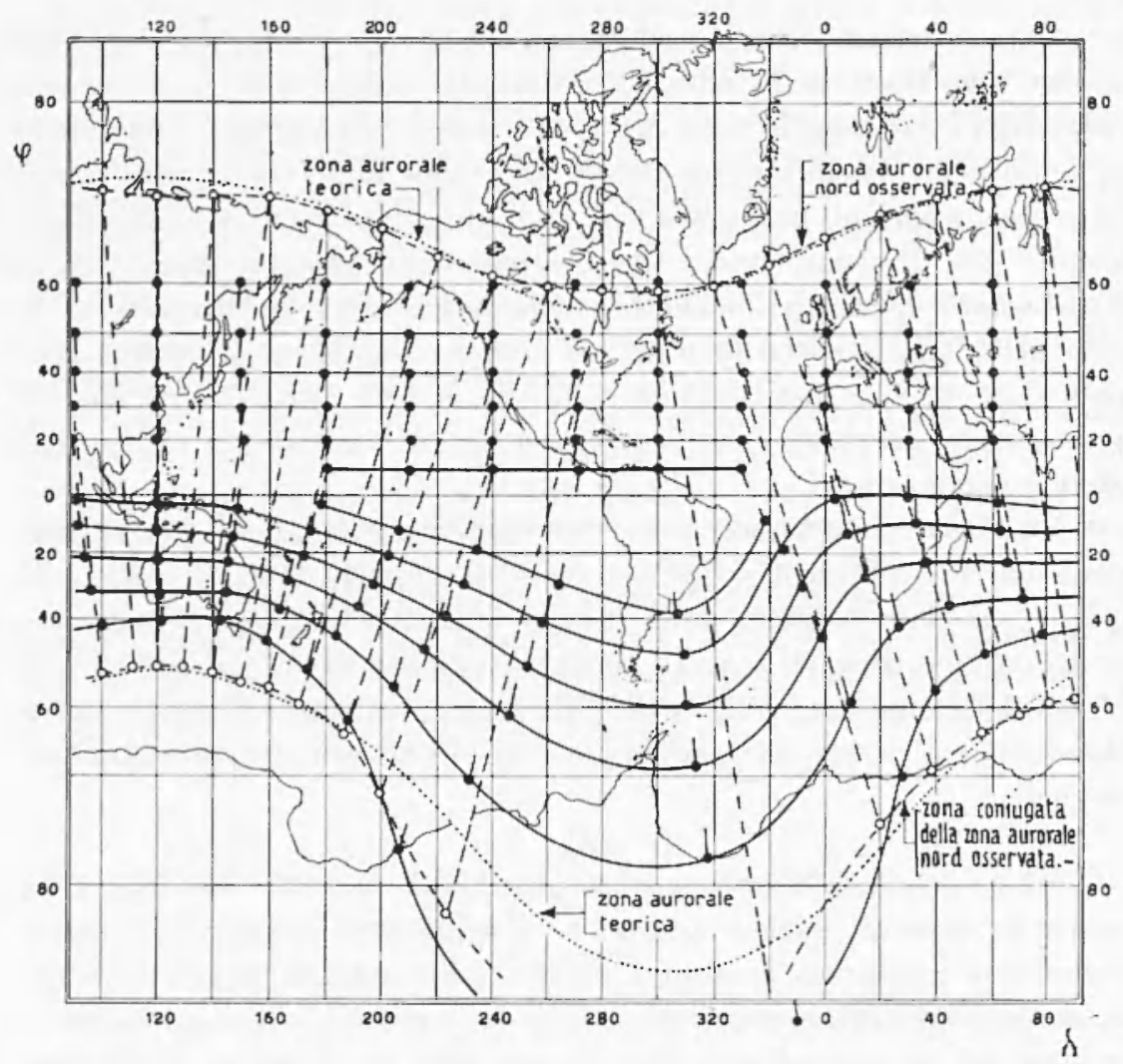

Fig. 5.I - Rappresentazione dei punti magneticanente coniugati, intersezione delle linee di forza del campo magnetico terrestre con la superficie della Terra; $p$ e $\lambda$, latitudine e longituline geografiche (secondo Testine e sibley).

finamento delle misure d'intensità dei raggi cosmici ha portato a taluni risultati inattesi. In particolare, Simpson e coll. $\left({ }^{2}\right)$, in base a misure di intensità della componente nucleonica e della componente mesonica, definito un "equatore geomagnetico dei raggi cosmici " come la linea luogo dei punti di minima intensità, hamno ottenuto un equatore corrispondente a un dipolo di momento e inclinazione rispetto all'asse di rotazione terrestre identici a quelli desunti dall'analisi di Gauss, rotato 
però verso orest di 40-45 (fig. 5.2). Il conseguente cambiamento delle coordinate geomagnetiche permette d'interpretare parecchie anomalie della intensità e dello spettro delle particelle $\alpha$ e dei nuclei pesanti primari.

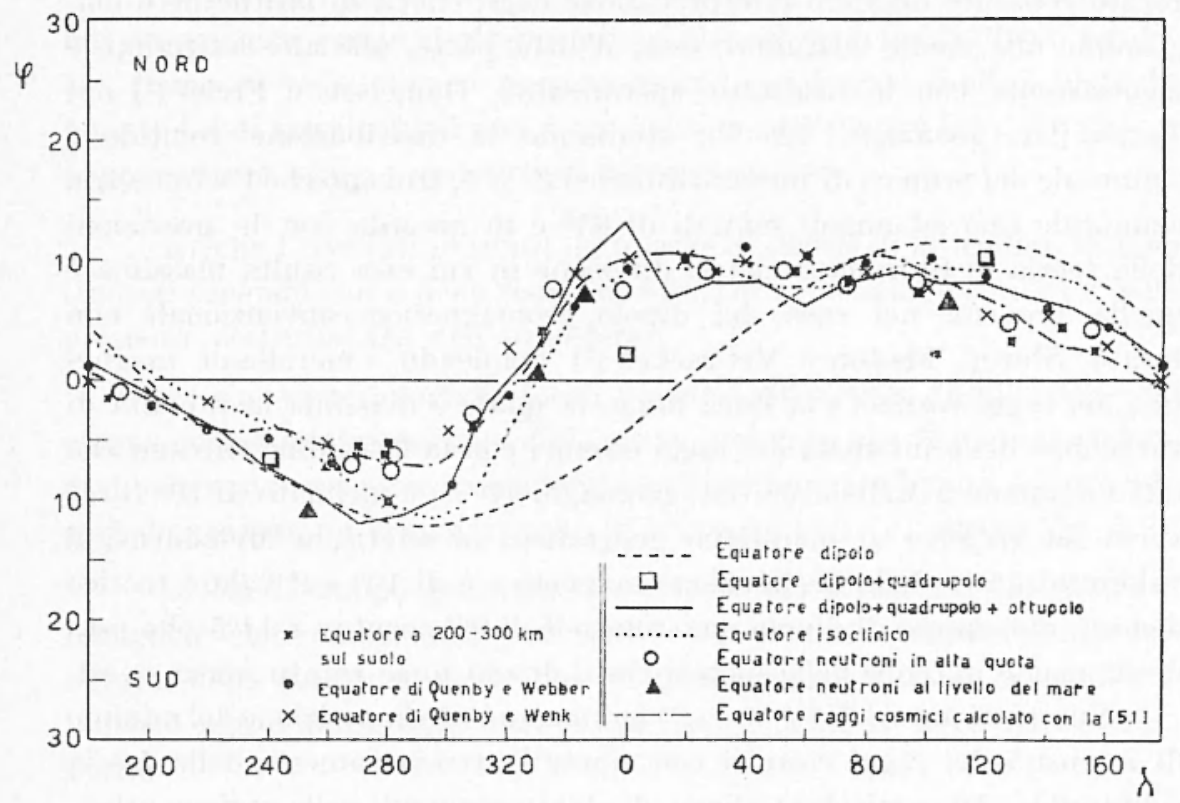

Fig. 5.2 - Andamento dellequatore magnetico terrestre secondo le diverse definizioni; $\varphi$ e $\lambda$. latitudine e longitudine geografiche (secondo Kellogg e schwartz; Rothwell; Vernov e coll., Quenhy e Webher: Quenby e Wenk).

Altre discrepanze rispetto al modello di dipolo geomagnetico sono messe in evidenza da ArcDonald (3), il quale confronta l'energia di taglio $T$ (in $\mathrm{MeV} / \mathrm{nucleone}$ ) per particelle a misurata sotto mo spessore residuo di atmosfera di $6 \mathrm{~g} / \mathrm{em}^{2}$ e alla latitudine geomagnetica $\Phi_{o}$ di $52-53^{\circ} \mathrm{con}$ la corrispondente energia di taglio prevista dalla teoria di Störmer (v. tabella 5.1) e determina la latitudine "effettiva " $\Phi^{\prime}$ richiesta dai valori misurati di $T$.

Tahella 5.1

\begin{tabular}{|c|c|c|c|}
\hline$\Phi_{0}$ & $T$ prevista & $T$ misurata & $\Phi^{\prime}$ \\
\hline $52^{\circ} 16^{\prime}$ & 455 & $225 \pm 25$ & $56^{\circ} 30^{\prime}$ \\
$52^{\circ} 23^{\prime}$ & 445 & $220 \pm 30$ & $56^{\circ} 23^{\prime}$ \\
$53^{\circ} 10^{\prime}$ & 400 & $160 \pm 15$ & $58^{\circ} 10^{\prime}$ \\
\hline
\end{tabular}


Non tutti i risultati sperimentali si inquadrano nel modello di dipolo rotato: Rose, Fenton, Katzman e Simpson $\left({ }^{4,5}\right)$, in ma serie di misurazioni estese dall'Artico all'Antartico, rilevano che se l'ipotesi del dipolo rotato consente migliore interpretazione degli effetti di latitudine e longitudine alle medie latitudini, essa, d'altra parte, alle alte latitudini, is inconsistente con le risultanze sperimentali. Danielson e Freier ${ }^{6}$ ) nel Texas (lat. geomagn. 420 $\mathrm{N}$ ), sturliando la distribuzione zenitale e azimutale dei primari di numero atomico $Z \geqslant 5$, trovano che l'asimmetria azinutale fino ad angoli zenitali di $65^{\circ}$ de in accordo con le previsioni della teoria di Störmer e che la direzione in cui essa risulta massima è quella prevista nel caso del dipolo geomagnetico convenzionale non rotato. Storey, Fenton e MaCracken (7) definendo "meridiano magnetico dei raggi cosmice "la linea lungo la quale è massima la latpidità di variazione della intensità dei raggi cosmici con la latiturline trovano che tale meridiano a Melbourne (lat. geomagne $47^{\circ}$ S) è inclinato di $10^{\circ} \pm 4^{\circ}$ verso est rispetto al mericliano geografico; in effetti, a Mrelbourne, i] valore misurato della declinazione magnetica d̀ di $10^{\circ}$ e il valore teorico dedotto dal campo di dipolo non rotato d di $9^{\circ} 5$, contro i $14^{\circ} 5$ che essa declinazione avrebbe nella ipotesi che il dipolo fosse rotato verso ovest.

Numerosi autori $(5,8,9,10,11,12)$ hammo sturliato la posizione del minimo di intensità dei raggi cosmicei con ripetuti attraversamenti della fascia equatoriale. Di particolare rilievo gli attraversamenti nella regione atlantica a ovest dell'Africa che è la regione dore maggiore è risultata la discelepanzal tra posizione di minima intensità dei laggei cosmici ed equatore geomagnetico del dipolo. Sul merirliano $14^{\circ}$ ovest il minimo corrisponde alla latitudine geografica di cilrea $7^{\circ} \mathrm{N}$; a questa longiturline, la latiturline prevista per il minimo differisce da quella prevista sia nel modello di dipolo rotato $\left(\sim 3^{\circ} \mathrm{N}\right)$ che nel modello di dipolo non rotato $\left(\sim 7^{\circ} \mathrm{S}\right)$, sia anche da quella prevista in base alle misurazioni di energia di taglio delle particelle $a$. Il punto di minimo risulta invece assai vicino all'equatore isoclinico (dip equator), definito come il luogo dei punti di inclinazione magnetica nulla al suolo.

Il problema della effettiva distribuzione del campo geomangetico a distanza i stato studiato da Pfotzer $\left({ }^{13}\right)$ sotto $u$ altro punto di vista: l'autore, partendo dall'osservazione che le discrepanze negli effetti di latitudine previsti pere i due casi di dipolo rotato e non rotato sono maggiori per particelle il cui spettro di rigirlità è più "ripido " di quello della radiazione cosmical normale, ha studiato la distribuzione geoglafica della intensita delle extraparticelle, principalnente protoni, che latggiunsero la Terra in occasione del brillamento del 23 febbraio 1956 (r. anche 
4.5.2). Sebbene i risultati ani Pfotzer perviene non possano ritenersi conclusivi, tuttavia gli efletti di latitudine dedotti dall'osservazione in 15 diversi punti della Terma sembrano, nel complesso, meglio in accordo con l'ipotesi del dipolo non rotato, nel senso che sebbene si abbiano sensibili discrepanze queste risultano minori ahe eol modello di dipolo rotato.

Come si rede, non si possono trame conclusioni molto rhiare in quanto i dati sperimentali non sono molto in aceordo tra loro. Ciò induce a prospettare varie possibilità di interpretazione:

a) che i risultati ottenuti da misure di effetti di latitudine vadano studiati tenendo conto della teoria di Störmer nella sua generalità e della presenza perturbatrice dellatmosfera;

b) che vaste anomalie locali possano alterare la distribuzione del (ampo geomagnetico esterno, cioc, più in generale, che lapprossimazione di dipolo non possa essere considerata suffieientemente buona, oceorrendo considerare termini di quadrupolo, di ottupolo, ece. (v. ancora fig. 5.2):

c) che il ampo geomagnetico estermo sia perturbato da effetti di induzione elettromagnetica provocati nela materia ionizzata interplanetaria dalla rotazione delle linee di forza geomagnetiche, la cui distribuzione non ì simmetrica rispetto allasse geografico di rotazione della Tellal.

Per approfondire la questione, consideriamo pio in rettaglio i punti in cliscussione: Winckler a Anderson ("4)), esaminando criticamente il significato fisice delle misure degli effetti di latitudine, richiamano l'attenzione sul fatto che in realtà l'uso di dispositivi ommidirezionali (camere di ionizzazione, ece.), la complicata distribuzione delle orbite permesse come pure i fenomeni di transizione nella atmosfera possono dar luogo a effetti apparenti anche sensibilnente diversi da quelli effettivi della ladiazione primaria; in particolare può aversi un "effetto di satnrazione" dei rivelatori in certe condizioni o in certe direzioni: cosi per es. ad alte quote (spessore residuo di atmosfera $\sim 10 \mathrm{~g} / \mathrm{cm} \mathrm{gm}^{2}$ la intensita della componente orizzontale dei raggi cosmiei si mantiene costante (15) nell'intervallo tra $51^{\circ}$ e $69^{\circ}$ di latitudine, mentre al contrario la componente rerticale risulta crescere del $45^{\circ} \%$ nello stesso intervallo di latitudini. Studiando sotto questa luce la posizione del "ginocehion" dell'efletto di latitudine, gli Sutori segnalano che le rigidità di taglio da essi osservate su palloni a varie quote, nell America settentrionale, compspondono a latitudini cirea to più alte di quelle geomagnetiche di dipolo, in rỏò acoordanclosi ron i citati risultati di McDonald. 
(Cir('a il punto b) sono assai interessanti i risultati di Rothwell $\left(^{16}\right)$, che la cercato una espressione empirica del momento di taglio pe per la componente nucleonica al lirello del mare: chiamato

$$
p_{D}=\frac{Z e}{4 c} R \frac{H_{D}}{\left(1+\frac{1}{4} \operatorname{tag}^{2} \delta_{D}\right)^{3 / 2}}
$$

il momento di taglio del campo del dipolo geomagnetico rentrato, con $R$ raggio della Terra, $H_{D}$ e $\delta_{D}$ componente orizzontale e declinazione appunto calcolate nel modello di dipolo, e definemdo con

$$
p_{s}=\frac{Z e}{4 c} R \frac{H .}{\left(1+\frac{1}{4} \operatorname{tag}^{2} \delta_{s}\right)^{3 / 2}}
$$

un analogo momento nel quale $H_{s}$ e $\delta_{s}$ rappresentano gli effettivi valori al suolo, nel luogo di osservazione, della componente orizzontale del campo a della declinazione, Rothwell pone la relazione

$$
p_{c}=x p_{s}+(1-x) p_{D}\left(\frac{p_{D}}{p_{s}}\right)
$$

ove $x$ is una quantità che va opportunamente determinata per confronto con i rlati sperimentali.

Paragonando i momenti di taglio per particelle a misurati da Rothwell stessa e da numerosi altri autori, in parte sopra citati, con la [ŏ.1] i risultato per $x$ un valore costante 0,88 a tutte le latitudini geomagnetiche tra $55^{\circ}$ nord e $47^{\circ}$ sud.

Ta linea di minima intensità della componente nucleonica dedotia dalla [5.1] con $x=0,9$ e mostrata in fig. 5.2.

Il lavoro di Rothwell suggerisce che le particolarità segnalate dai vari autori siano sopratutto determinate dalle differenze locali del campo magnetico "reale " da quello geomagnetico convenzionale. In effetti la deflessione dei raggi cosmici per opera del campo magnetico terrestre avviene nella sua maggior parte a distanza non superiore a qualche raggio terrestre; ciò giustifica perchè differenze tra l'effettivo campo magnetico terrestre e quello convenzionale di dipolo, che sono apprezzabili solo fino a distanza dell'orline delle migliaia di $\mathrm{km}$, abbiano tuttavia effetti rilevanti sui raggi cosmici.

In forma più generale, il problema della effettiva distribuzione del compo magnetico intorno alla Terra va studiato, come già si è detto, includendo nella analisi in serie di armoniche sferiche un numero di 
termini sufficientemente elevato: con tale procedura la distribuzione del campo viene a perdere la simmetria circolare propria del modello di dipolo.

In particolare, Kellogg e Schwartz (17) hanno preso in considerazione l'effetto dei termini di quadrupolo e di ottupolo, mentre Quenby e Webber ${ }^{(11)}$ hanno usato i primi 48 termini dello sviluppo in serie; Quenby e Wenk $\left(^{18}\right)$ più recentemente hamno migliorato le approssimazioni di

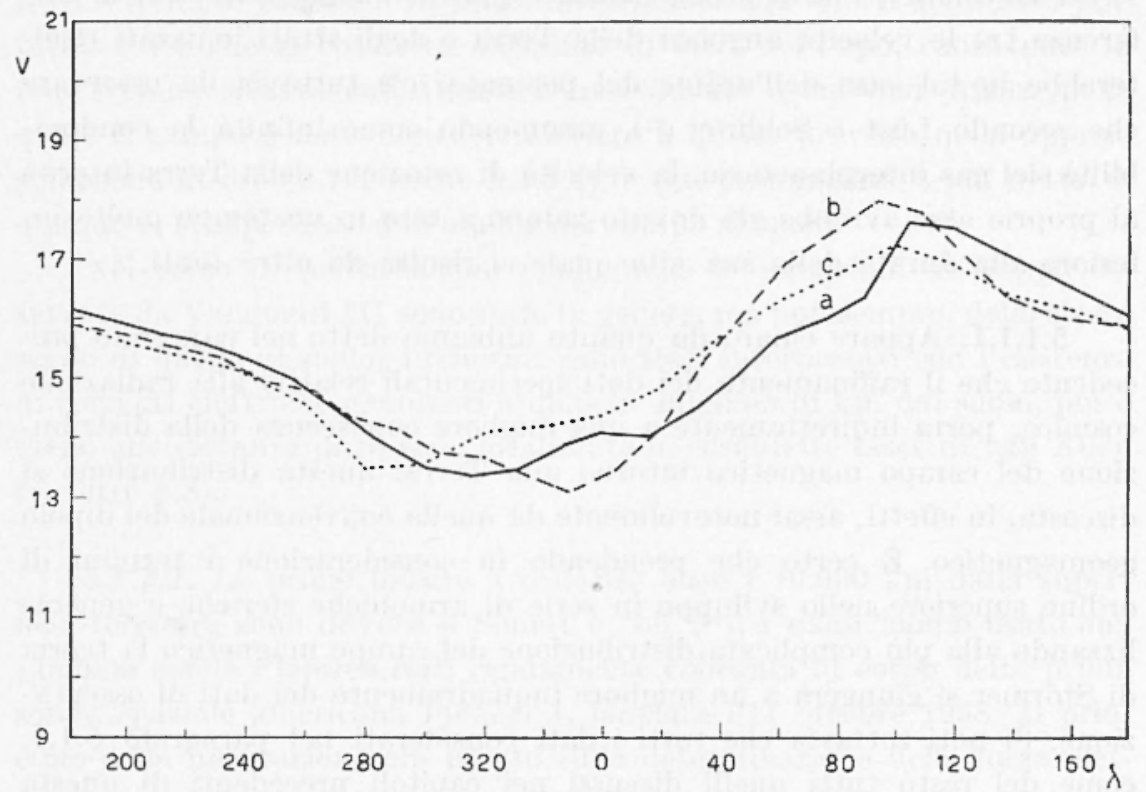

Fig. 5.3 - r, rigiditì magnetica di taglio (in $G \mathrm{~F}$ ) per protoni in direzione verticale allequatore magnetico, secondo Kellogg e Schwartz (curva a), Quenby e Webber (curva b) e Quenby e Wenk (curva $c$ ); i. longitudine greografica.

Quenby e Webber, in particolare prendendo in ronsiderazione gli effetti di " penombra " della Terra. Ise ligure 5.2 e 5.3 riportano gli andament. calcolati dai suddetti autori per l'equatore magnetico, definito come luogo dei punti nei quali è massima la rigidità di taglio pes particelle dei raggi cosmici incidenti verticalmente, nonché i valori di questa rigidità in corrispondenza dell'equatore stesso.

Diverso è il punto di vista degli autori che attribuiscono le amomalie di distribuzione della intensità dei raggi cosmici a cause esteme alla Terra che provocano distorsioni del campo di dipolo. In particolare, Beiser ${ }^{(99}$ ) attribuisce la distorsione del campo geomagnetico di dipolo all'effetto 
delle correnti indotte dal movimento del dipolo magnetico terrestre, inclinato rispetto all'asse di rotazione della Terra, sulla materia interplanetaria ionizzata.

Inglaham $\left(^{(20}\right)$ sostiene che ma rotazione verso ovest del dipolo magnetico risulta semplicemente assumendo che gli strati ionizzati più esterni alla Terra restino indietro rispetto agli strati piu vicini, rotando con velocita angolare inferiore a questi; si noti che non si tratta degri strati ionosferici, ma di strati distanti qualche raggio terrestre; la differenza tra le velocita angolari della Terra e degli strati ionizzati risulterebbe in tal caso dellordine del pereento; c'è tuttavia da osservare che secondo Lïist a Schluter (21), assumendo come infinita la conducibilita del gas interplanetario, la velocità di rotazione della Terra intomo al proprio asse arrebbe già dovuto richursi a zero in un tempo molto inferiore alla durata della sua vita quale ci risulta da altere fonti.

5.1.1.1. Appare chiaro da quanto abbiamo detto nel paragrafo precerlente che il raffinamento dei dati sperinnentali relativi alla raliazione cosmion, porta indirettamente a ma migliore conoscenza della distribuzione del ampo magnetico intorno alla Terra: questa distribuzione si discosta, in effetti, assai notevolmente da quella convenzionale del dipolo geomagnetico. E certo che prendendo in considerazione i termini di ordine superiore nello sviluppo in serie di armoniche sferiche e generalizzando alla più complicata distribuzione del campo magnetico la teoria di Störmer si giungerà a un migliore inquadramento dei dati di osservazione. Si noti tuttavia che tutti i dati considemati nel paramgafo 5.1.1, come del resto tutti quelli discussi nei capitoli precedenti di questa rassegna, sono relativi a osservazioni fatte al suolo o a poca distanza dal suolo. Ne consegue la necessita di completare e perfezionare il quadro fin qui costruito con i risultati di misurazioni dirette del campo magnetico terrestre fino a distanza di parecehi raggi terrestri.

5.1.2. Wisure dirette di compi magnetici nella esosfera. - Di grande interesse sono le misure dirette del campo magnetico nello spazio intorno alla Terra, divenute possibili in questi ultimi anni mediante strumenti portati da satelititi e sonde spaziali.

Si tratta ancora di risultati pinttosto sporadici e preliminari, alcuni dei quali tuttavia consentono già, come verlremo, di acquisire informazioni di grandissina importanza sperimentale e teorica.

5.1.2.1. A parte le misure di ammo magnetico effettuate da vali antori nella bassa ionosfera, nell'intento di studiare il sistema di correnti 
plettriche cireolante nello strato $E$, considerato rome la calusa delle variazioni diurne del eampo magnetico terrestre al suolo, le prine misure dirette, a quote fino a $1000 \mathrm{~km}$, sono quelle di Dolginor a coll. (22) su Sputnik III (1958 $\left.\delta_{2}\right)$ nel periodo 15 maggio-5 giugno 1958. Successive misure, più precise e distribuite ne] tempo su un periodo di circa tre nesi. a distanze dal suolo tra 510 e $3550 \mathrm{~km}$ nell’intervallo di latitudini geografiche tra $33^{\circ}$ nord e sud si debbono a Vanguard III (1959 7$)$, lanciato il 18 settembre 1959 . Un risultato interessante agli effetti del nostro problema ( $\left.{ }^{23}\right)$ i che la cosideta anomalia di Città del Capo, consistente in una regione situata sull Atlantico meridionale e sul Sucl Africa nella qua e il campo i noterolmente inferiore a quello previsto nella approssimazione di dipolo ( $r$ anche 5.3.5.4), è più pronunciata e più netta di quando ci si aspettasse dallanalisi del campo al suolo.

Nel corso di perturbazioni magnetiche le valiazioni di campo osservate da Vanguard III sono state in genere, ma non sempre, dello stesso segno di quelle al suolo; i risultati sono stati interporetati con l'esistenza di comenti elettriche circolanti a qualche migliaio di kim dal suolo, più o meno alle distanze dove sono localizzate le cosiddette fasce di van Allen (1. oltre 5.3$)$.

5.1.2.2. Le prime misure a distanze oltre i $10.000 \mathrm{~km}$ dalla superficie terrester sono dovate a Sonett e coll. (2-4), i quali hanno usato ma "bobina sonda " (search coil) rigidamente collegata al colpo della prima sonda spaziale americana Pioneer I, lanciata l'11 ottobre 1958. Il principio della misurazione era basato sulla determinazione della forza elettromotrice indotta nella bobina per effetto della sua rotazione intormo all'asse stabilizzato di rotazione del veicolo: reniva in tal modo misurata la componente $B \perp$ del campo geomagnetico secondo il piano normale allasse di rotazione della bobina. Tale asse risulto fortuitamente, dutante molta parte del tempo di misura, quasi perpendicolare alla direzione del canpo geomagnetico $B$ previsto nel modello di dipolo: la misura di $B$. L rappresenta quindi un ralore assai prossimo a quello di $B$.

I valori misurati si riferiseono agli intervalli di distanze geocentriche di $3,7 \div$ i e $12,3 \div 14,8$ raggi terrestri (rispettivamente $(2.4 \div 4,4) \cdot 10^{4}$ e $(\pi, 8 \div 9,-1) \cdot 10^{1} \mathrm{~km}$; il periodo di misura cade in ma epoea di eccezionale tranquillità geomagnetica (indice magnetico massimo $A_{p}=5$ ).

Nel primo intervallo di clistanze i ralori misurati di $B_{\perp}$ e quelli previsti nel modello di un dipolo ecerentrico sono in aceordo, a parte lievi fluttuazioni di ampiezza non superiore a qualehe \%. Soterolmente diversa è invere la situazione a distanze oltere i 10 raggi terestri. I ralori 
misurati si discostano fino al $100 \%$ dai valori previsti (fig. 5.4); per di più essi presentano una diminuzione con la distanza geocentrica $r$ che, rispetto a quella prevista (del tipo $r^{-3}$ ), ì sensibilmente più rapida (del tipo $r^{-5}$ ).

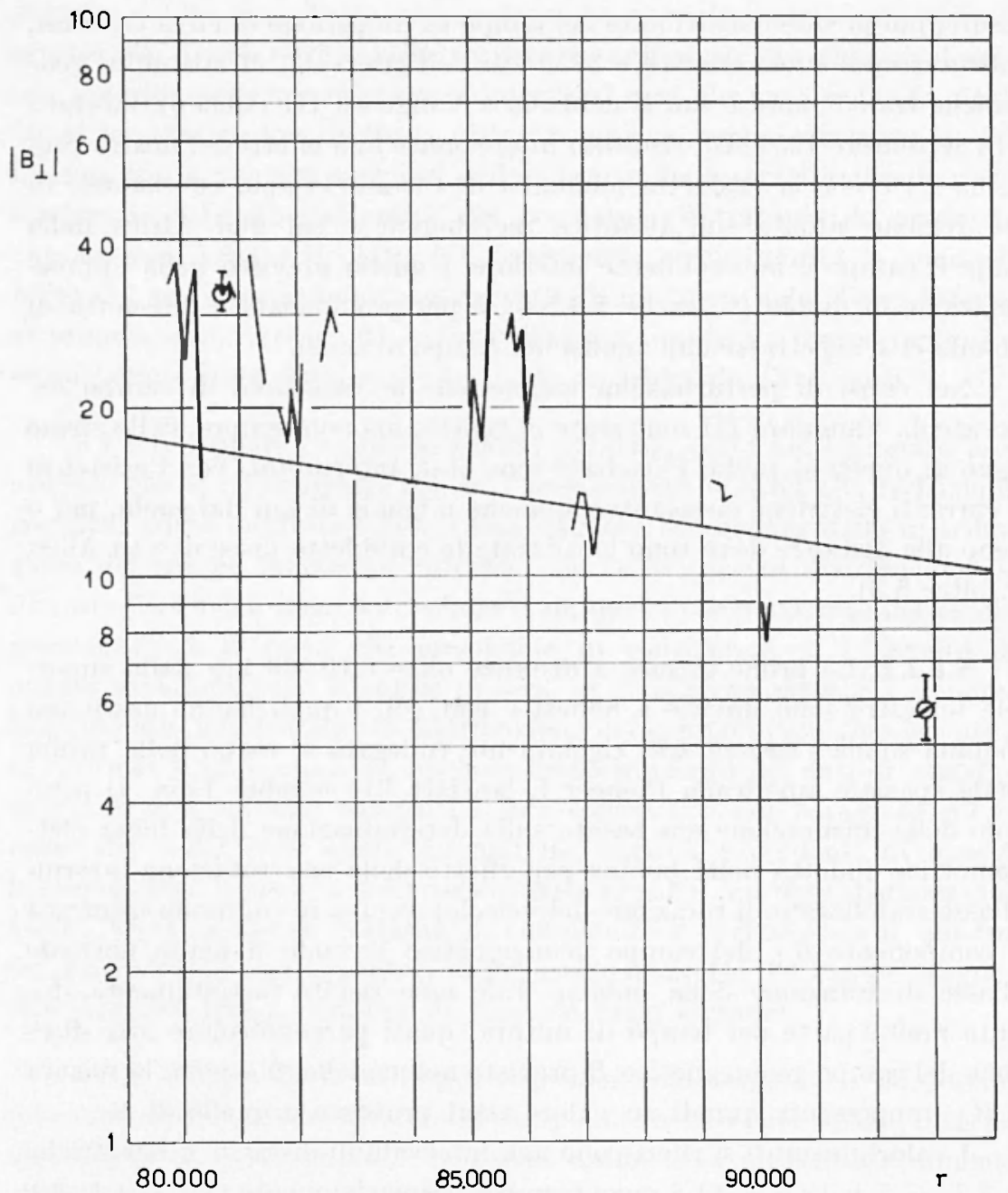

Fig. 5.4 - Andamento della componente $B_{\perp}$ (in gamma); $r$, distanza georentrica in $\mathrm{km}$; la linea continua rappresenta landamento ealcolato per il campo di dipolo (secondo Sonett, Smith e Sims).

Risulta anche ben evidente (fig. $\check{5} .5 a$ ) la presenza di piceole fluttuazioni quasi periodiche con "periodo" dell'ordine dei secondi, fluttuazioni rhe non sono presenti invece a distanze inferiori a 7 raggi terrestri. 

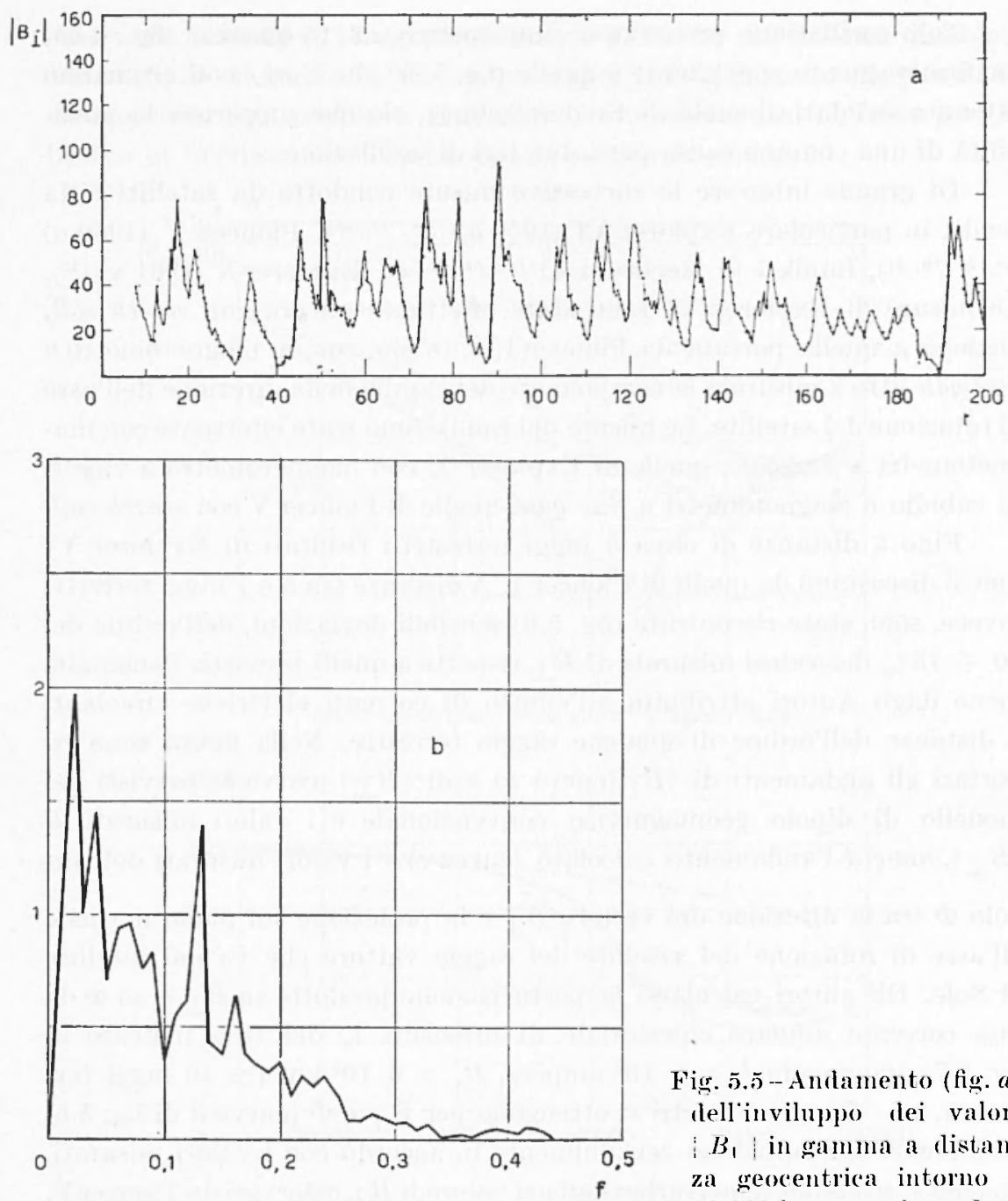

Fig. 5.5-- Andamento (fig. $a$ ) dell'inviluppo dei valori $B_{1}$ in gamma a distanza reocentrica intorno a $80.000 \mathrm{~km}$ e corrisponden -

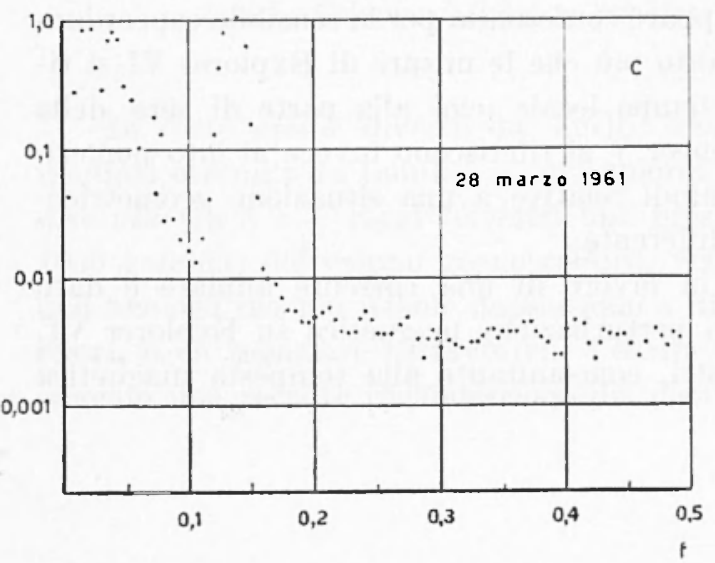
te spettro differenziale della poten\%a associata alle piceole oscillazioni di $B_{\perp}$ in unità $10^{-6}$ grauss $^{2} \cdot 11 z^{-1}$ (fig. b): per confronto in figr. $c$, spettro differenziale della potenza (in mita $10^{-10}$ gauss $\left.^{2} \cdot \mathrm{Hz}^{-1}\right)$ a Fre dericksburg: 1 , tempo in secondi: $f$. frequenza in $\mathrm{Hz}$ (secondo Sonett, Judge, Sims e Kelso: Ness, skillman, scearce e lleppner). 
Tali oscillazioni presentano mo spettro di frequenza (fig. 5.5̌b) qualitativamente somigliante a quello (tig. 5.5e) che Xess e coll. ( ${ }^{25}$ ) hanno ottenuto sui dati al suolo di Fredericksburg, cio che sugrgerisce la possibilità di una comme causa per i due tipi di oscillazione.

Di grande interesse le suceessive misure condotte da satelliti e da sonde, in particolare Explorer VI $\left(1959 \delta_{1}\right)\left({ }^{26,}{ }^{27,}{ }^{28}\right)$, Pioneer $\mathrm{V}(1960 a)$

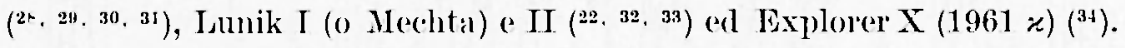
Le misure di Explorer VI sono state eflettuate ancola con search coil, analoga a quella portata da Pioneer I, e, in più, con un magnetometro a flux gate at to a misurare la componente del campo nella direzione dellasse di rotazione del satellite. Ise misure dei Ismik sono state effettuate con magnetometri a flux gate, quelle di Explorer $\mathrm{X}$ con mangetometro a vapori di rubidio e magnetometri a flux grate, quelle di Pioneer $T$ con search coil.

Fino a distanze di circa 5 raggi terrestri i risultati di Explorer VI non si discostano da quelli di Pioneer I. A distanze tra 5 e 7 raggi terrestri invere, sono state riscontrate (fig. 5.6) sensibili deviazioni, dell'ordine del $50-75 \%$ dei valori misurati di $B \perp$ rispetto a quelli previsti; l'anomalia viene dagli Autori attribuita alleffetto di correnti elettriche cireolanti a distanze dell'ordine di qualede raggio terestre. Nella figura sono riportati grli andamenti di $|B|$ (curva a) e di $|B| \mid$ (curva $b$ ) previsti nel modello di dipolo geomagnetico convenzionale e i valori misumati di $B \perp$ l, nonché l'andamento calcolato (curva $c$ ) e i valori misumati dell'an-

golo $\Phi$ tra la direzione del vettore $\vec{B} \perp$ e la proiezione sul piano normale all'asse di rotazione del satellite del ragrovio vettore che va dal satellite al Sole. Gili autori calcolano la perturbazione prodottasu $B \perp$ e $\operatorname{su} \Phi$ da ma corrente anulare equatoriale di intensità $I$, del tipo indicato in fig. 5.7 ; assumendo $I=5 \cdot 10^{6}$ ampère, $R_{0}=6 \cdot 10^{4} \mathrm{~km}(\simeq 10$ mggi terrestri), $a=3$ raggi terrestri si ottengono per $B_{\perp}$ e $\Phi$ (curve $d$ di fig. 5.6) andamenti ele appaiono sensibilmente in arcoordo con i valori misurati.

Se si studiano le perturbazioni nei valori di $B_{\perp}$ osservati da Pioneer $V$. la suddetta interpretazione appare confermata per la sensibile concordanza nei valori di $R_{0}$, a e $I$, tanto più che le misure di Explorer VI si riferiscono circa alle 2100 di tempo locale (cioè alla parte di sela della Terra), mentre quelle di Pioneer $Y$ si riferiscono invece al lato pomeridiano della Terla e sono quindi relative a una situazione geometrieoastronomical notevolmente differente.

Un ulteriore elemento in favore di una corrente amulare d dato dalla osservazione ${ }^{35}$ ) di una perturbazione magnetica su Explorer VI, a distanza di 4 ragrei terrestri, concomitante alla tempesta mannetion 
osservata al suolo il 16 agosto 1959 ma 2,5 volte più intensa (massima diminuzione di intensità di ampo: airea 350 gamma su Explorer VI contro circa 140 gammar a Irancayo): in altri termini l'effetto perturbativo si accresce avvicinandosi alla corrente anulare.
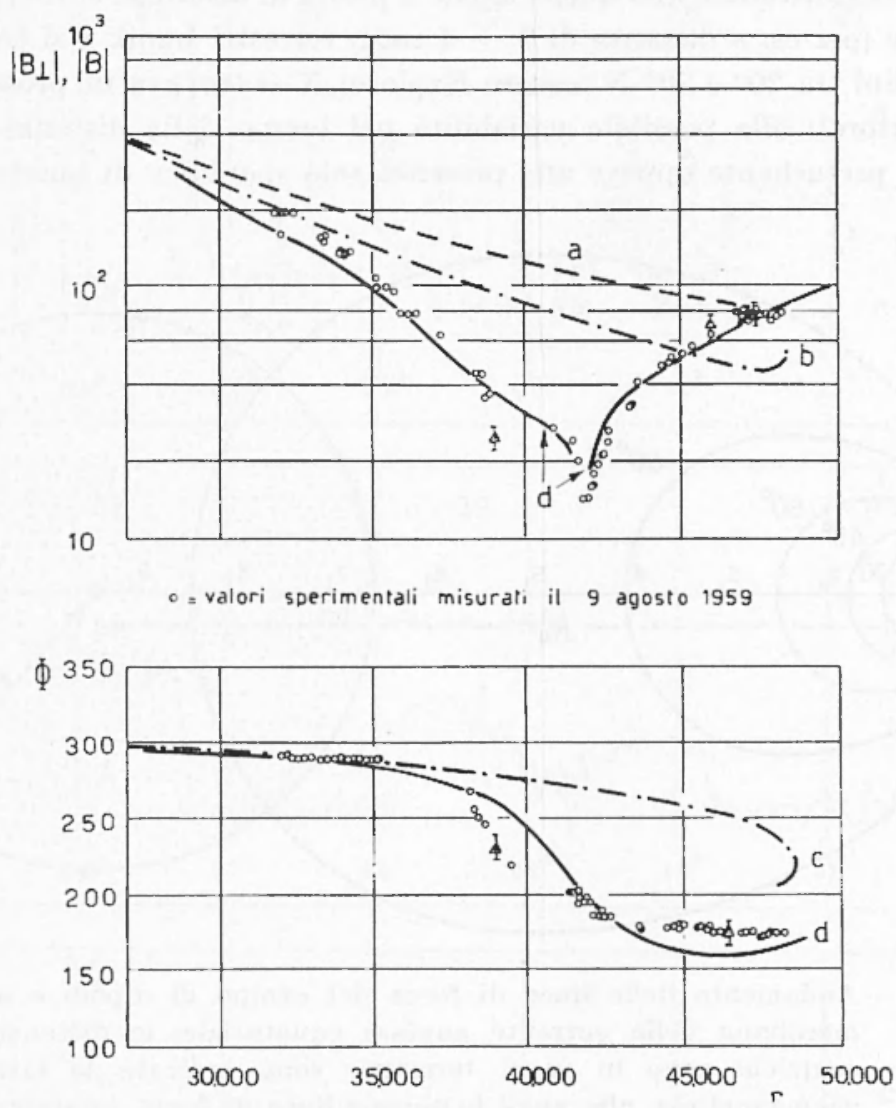

lig. 5.6 - $\mathrm{B}_{\perp}$ e $\mathrm{B}$. in gamma: $r$, distanza geocentrica in $\mathrm{km}$ (secondo smith. Coleman. Judge e Sonett).

In reeto grado diversi da quelli soprar riportati sono invece i risultati ottenuti da Ismik I e da Explorer X. Il primo ha osservato, a distanza tra 3 e 4 raggi terrestri una grossa depressione fino a circa 1000 gamma) del campo geomagnetico. Explorer $\mathrm{X}$ ha invere rivelato una analoga ma più debole depressione a distanza tra 1,6 e + raggi terrestri; è da segnalaue tuttavia che l'entita di quest'ultima depressione, secondo una recente rielaborazione dei dati $\left({ }^{36}\right)$, si è ridotta da qualehe 
centinaio a qualche decina di gamma. In ambedue i casi gli autori pensano allefefetto di correnti anulari equatoriali.

Le sensibili differenze tra le distanze alle quali sono presenti tali anomalie del campo magnetico possono attribuirsi a diverse ragioni: alla diversa latitudine alla quale, anche a parità di distanza, si riferiscono le misure (per es. a distanza di $3 \div 4$ raggi terrestri Lunik I si trovava a latitudini tra $20^{\circ}$ e $30^{\circ} \mathrm{N}$ mentre Explorer $\mathrm{X}$ si trovava in prossimità dell'equatore); alla possibile valiabilità nel tempo della distanza della corrente perturbante oppure alla presenza solo sporadica di questa.

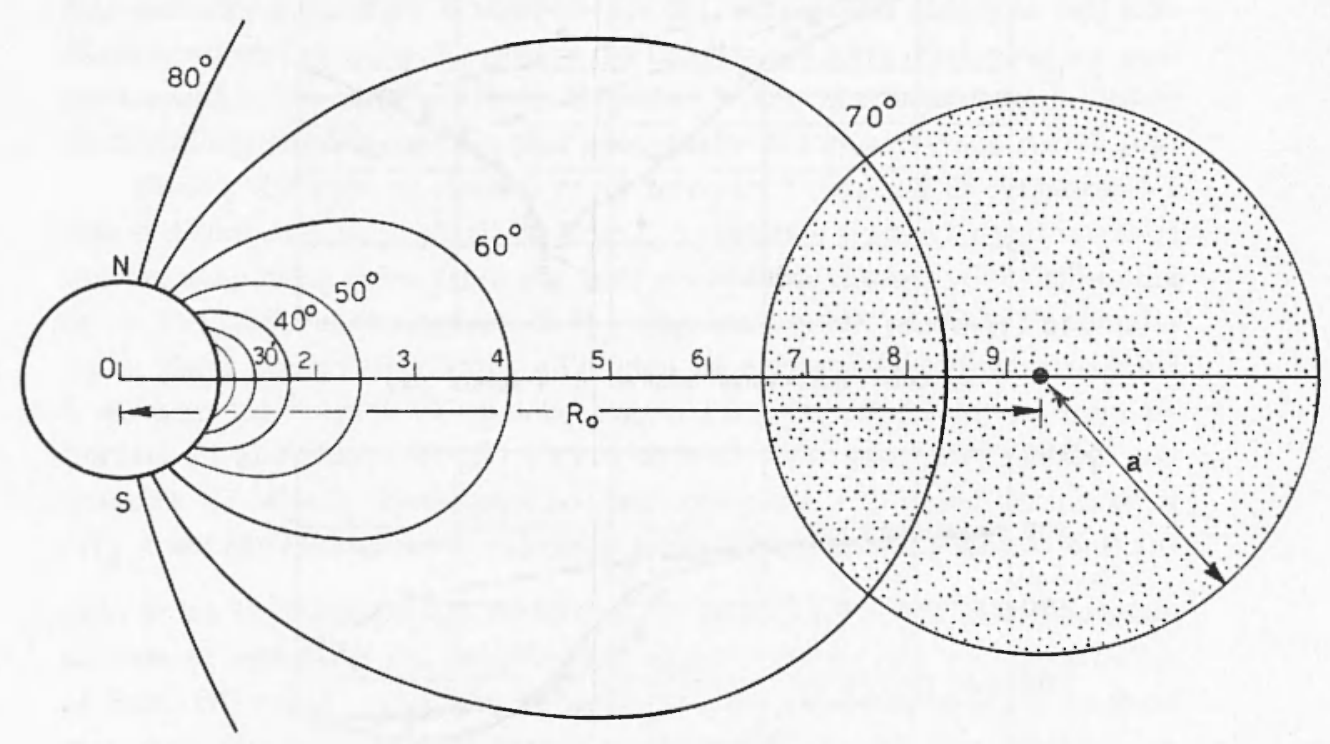

Fig. 5.7 - Andamento delle linee di forza del campo di dipolo e sezione meridiana della corrente anulare equatoriale; le distanze geocentriche sono in raggi terrestri: sono indicate le latitudini geomagnetiche alle quali le singole linee di forza intersecano la superficie terrestre (secondo Smith, Coleman, Judge e Sonett).

Una conferma indiretta della presenza di ma corrente anulare comes suggerita dalle misure di Explorer VT e Pioneer V viene data dalle misure di flusso di particelle di energia maggiore di circa 200 eV compiute dai Lunik a distanze tra circa 9 e 12 raggi terrestri. I flussi misurati sono

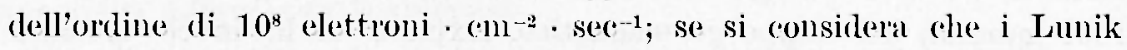
tagliano il piano equatoriale geomagnetico a cirea $60.000-65.000 \mathrm{~km}$ dalla Terra e la corrente anulare postulata per interpretare le misure magnetiche di Explorer VI e Pioneer V fluisce tra circa 40.000 e $80.000 \mathrm{~km}$, il flusso 
elettronico misurato può essere proprio quello al quale attribuire la corrente anulare. Come ordini di grandezza un flusso di $10^{8}$ elettroni $\cdot \mathrm{cm}^{-2}$. $\mathrm{sec}^{-1}$ corrisponde (su una sezione di diametro di $15.000 \mathrm{~km}$ ) a correnti
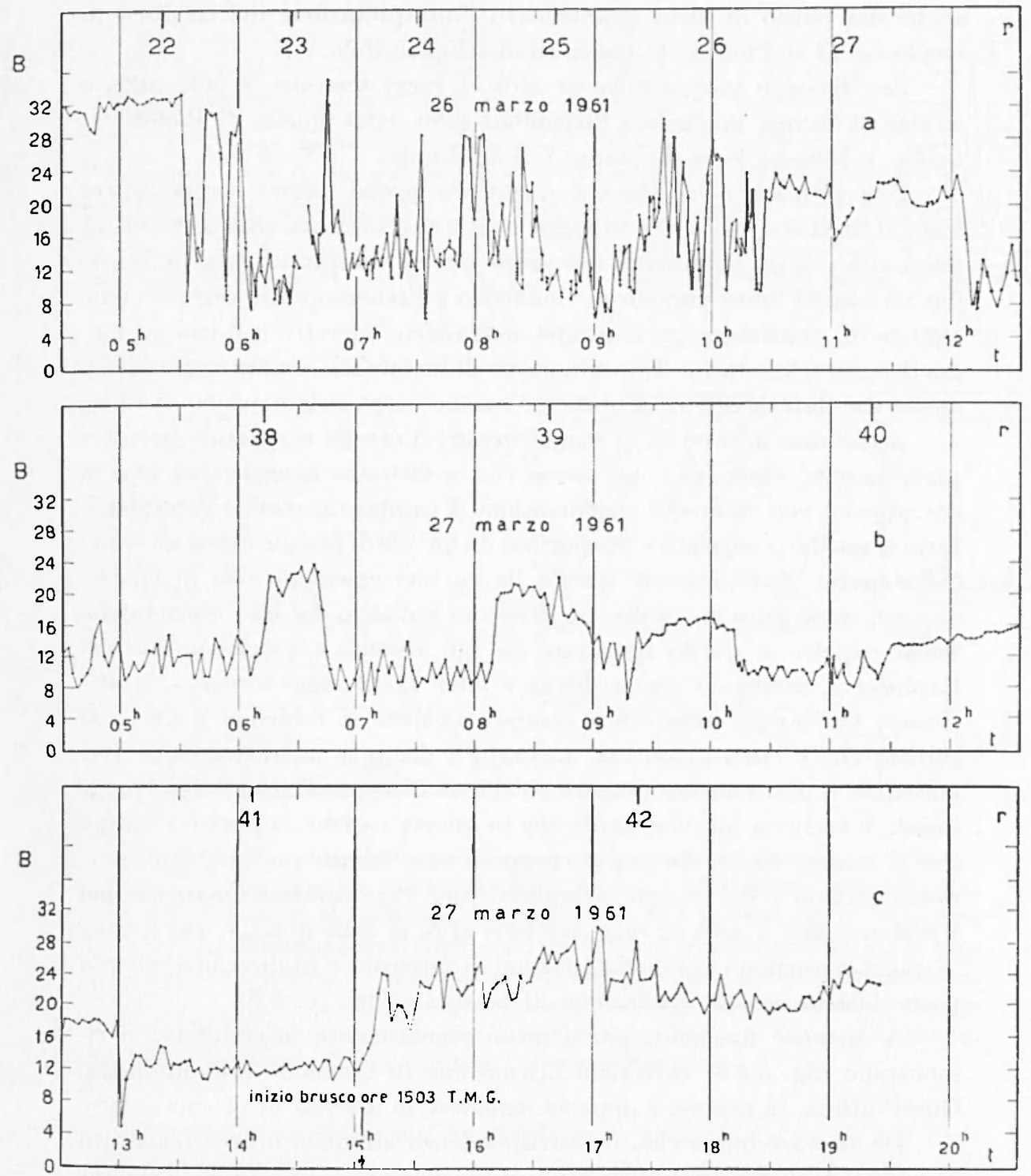

Fig. $5.8-b$, intensità del campo magnetico in camma: $r$, distanza geocentriea in raggi terrestri; $t$, tempo medio di Greenwhich (se. condo Heppner, Ness, Skillman e Scearce). 
dell'ordine di $10^{7}$ ampìre; tenendo conto del fatto che l'anomalia magnetica osservata va attribuita alla componente di tale corrente perpendicolare alle linee di forza del campo magnetico terrestre, si deduce che anche dal punto di vista quantitativo l'interpretazione dei risultati di Explorer VI a Pioneer V possa ritenersi plausibile.

Per distanze geocentriche di oltre 7 raggi terrestri le sole misure dirette di campo magnetico disponibili sono, oltre quelle di Pioneer I, quelle di Pioneer V, di Explorer $\mathrm{X}$ e dei Isunik.

Ena prima conclusione che da queste poche misure si può trarre i che a distanze tra i 7 e i 13 lageri terrestri si instauma una certa instabilita del campo magnetico nel senso che si osservano caratteristiche fluttuazioni di breve periodo che inducono a ritenere questa regione come regione di transizione tra il campo magnetico terrestre e il campo magnetico interplanetario. Tale situazione di instabilita sembra risperehiata anche dai dati di intensità della radiazione corpuscolare (v. fig. 5.1. a).

A distanze intorno ai 13 raggi terrestri il campo magnetico terrestre praticamente "termina ", nel senso che a distanze magroiori ad esso si sovrappone con intensità confrontabile il campo magnetico interplanetario o quello "congelato" trasportato da nuvole di plasma che si spostano nello spazio. Naturamente questo linite può essere diverso in epoche diverse, come pure Iungo diverse direzioni radiali o dal lato diurno della Terra rispetto a quello notturno. Ie più recenti e precise misure di Explorer $\mathrm{X}$ mostrano che, a distanze oltre 11-12 raggi terrestri, le differenze tra campo misurato e campo calcolato si riducono a $20 \div 30$ gamma che ì circa l'intensità misurata a distanze assai magroiori. Pur potendosi a priori ancora pensare all'effetto di appropriate correnti equatoriali, i tuttavia più verosimile che in questa regione appunto il campo che si misura risulti da una sovmapposizione del campo magnetico terrestre residuo e del campo interplanetario. Tale carattere permane poi a distanze fino a circa 22 raggen terrestri al di la delle quali, e fino a circa 27 raggi compaiono rapide fluttuazioni di intensità e di direzione, press'a poco simultanee alla rivelazione di plasma solare (v. 5.5).

A distanze maggiori, più o meno regolamente intervallate, si riscontrano (fig. 5.8b) valiazioni discontinue di direzione e di intensità. Quest'ultima, in genere, i apparsa maggiore in assenza di plasma solare.

Da rilevare infine che, in corrispondenza all'inizio brusco registrato al suolo il 27 marzo 1961 alle 1503 T.M.G., il campo misumto da Explorer $\mathrm{X}$ ha preso a crescere dal livello medio di $\sim 10$ gamma a oltre 20 gamma (fig. 5.8 c) con effetto massimo di variazione di intensità tra 7 e 9 minuti dopo l'inizio brusco sulla Terra. 
A fini di rhiarezza è da dire che la distanza di Explorer $\mathrm{X}$ dal Sole era di $200.000 \mathrm{~km}$ maggiore della distanza Terra-Sole: la perturbazione sembra cosi propagarsi su tale lunghezza nel tempo di qualche minuto.
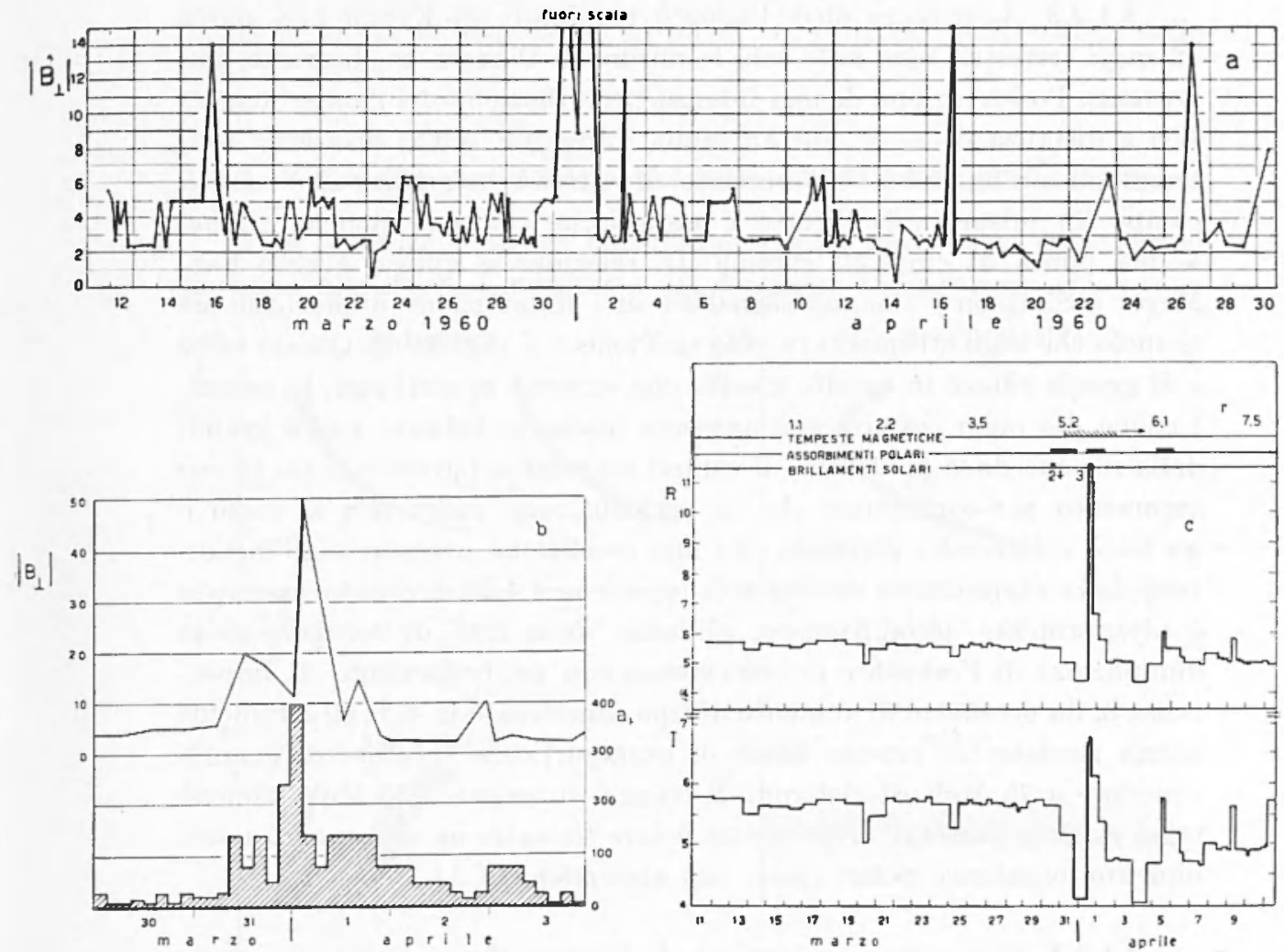

Figr. 5.9 - $\mid B_{1} !$, componente normale del campo magnetico in gamma (firr. $a$ e $b$ ): nell'istogranma di fig. $b$ andamento dell'indice $a_{k}$. a Fort Belvoir (lat. geomagn, 50,10 $\mathrm{N}$ ): $I$, intensità di conteggio di coincidenza triple su Pioneer $V$ in impulsi/sec (fig. $c$ ): $R$, rapporto tra $I$ e la intensità della componente nucleonica misurata al suolo: $r$, distanza geocentrica in milioni di km: le date sono in tempo universale (secondo Coleman, Davis e Sonctt: Coleman. Sonett, Judge e smith: Meyer e sinjuson).

Sebbene i dati raceolti da Explorer $\mathrm{X}$ abbiano un valore per certi versi limitato dalla loro complicata dipendenza dal tempo e dalla posizione nello spazio, tuttavia essi semblano sugrgeriere alcume possibilità di grande interesse teorico: rhe nello spazio si abbia effettivamente una propagazione delle perturbazioni magnetiche, quelle a inizio bruseo al- 
meno, e che, d'alta parte, variazioni anche intense del campo magnetico esterno non appaiono associate ad apprezzabili variazioni del campo magnetico misurato al suolo, almeno a basse a medie latitudini.

5.1.2.3. A distanze oltre l'apogeo rageinnto da Explorer X (circa 42 raggi terrestri) sono note solo le misure di Pioneer $V$ : di grande importanza l'osservazione di una intensa perturbazione del campo magnetico a distanza di $\sim 5.10^{6} \mathrm{~km}$ dalla Terra (fig. 5.9 a) pressoché cont(mpolanea (fig. 5.9b) all'aumento di attività magnetica al suolo; il campo $B \perp$ misurato da Pioneer V passa da un valore di qualche gamma a una punta di circa 50 gamma. In relazione a questo erento Fan, Iryer e Simpson (37) hanno segnalato una diminuzione di Forbush sia al suolo che sugli strumenti in rolo su Pioneer $\mathrm{V}$ (fig. 5.9 c). Questo fatto è di grande rilievo in quanto mostra che, almeno in certi casi, la perturbazione dei raggei cosmici può arvenire anche a distanze molto grandi dalla regrione dove preslomina il campo magnetico terrestre: è un nuovo argomento per confermare che la perturbazione magnetica al suolo is un fatto collaterale, piuttosto che una comdizione necessaria all'instaurarsi della diminuzione di Forbush (v. anche 4.4.2). Lievento osservato ¿ ulteriomente aceompagnato, all"inizio della fase di recupero della diminuzione di Forbush e in coincidenza con un brillamento di importanza 3, da un effetto di ammento di tipo considerato in 4.5: gli strumenti hamno rivelato 10 intenso flusso di extraparticelle (protoni di energia superiore a 75 MIeV al elettroni di energia superiore a $15 \mathrm{MeV}$, nonchi raggi $\gamma$ ): pure presente nella calotta polare terrestre un aumento di assorbimento ionosferico polare (polar cap absorption, P(A).

5.1.2.4. Una prima conclusione di insieme che si può trarre dalle misure di eampo magnetico al suolo e nello spazio circostante la Terra che l'influenza del campo magnetico terrestre si astende in pratica lino a distanze di $70.000 \div 80.000 \mathrm{~km}$. Ad esso sono in ogni caso sorrapposti effetti doruti ai campi prodotti da correnti circolanti perpendicolarmente alle linee di forza del "ampo "statico" a distanze noterolmente valiabili, prossime o coincidenti con quelle delle fasce di radiazione di vall Allen (v. 5.3).

Scendendo a maggrore dettaglio si notano frequentemente situazioni perturbate del campo nello spazio esterno, non sempre associate ad analoghe perturbazioni al suolo a viceversa. Abbastanza collegate tra loro appaiono invece delle fluttuazioni di breve periodo sia al suolo che a distanza di parecehi ragei terestri plausibilmente dorute a onde idro- 
magnetiche: a questo riguardo anzi, da una prima analisi dei dati di Explorer $\mathrm{X}$ risulta (25) (he nel periodo di rapirle fluttuazioni iniziatosi intorno alle 0530 T.M.G. del 26 manzo 1961 (fig. 5.8 a), le variazioni hanno il calattere di un campo perturbatore che ruota perpendicolarmente a un campo statico, in un modo che is quello caratteristico delle onde di Alfven.

\subsection{Fenomeni elettromagnetici nello spazio intorno alla Terra.}

Una noterole fonte di informazione sullo stato fisico dello spazio prossimo alla Terra è costituita dai fenomeni radioelettriei rhe vanno
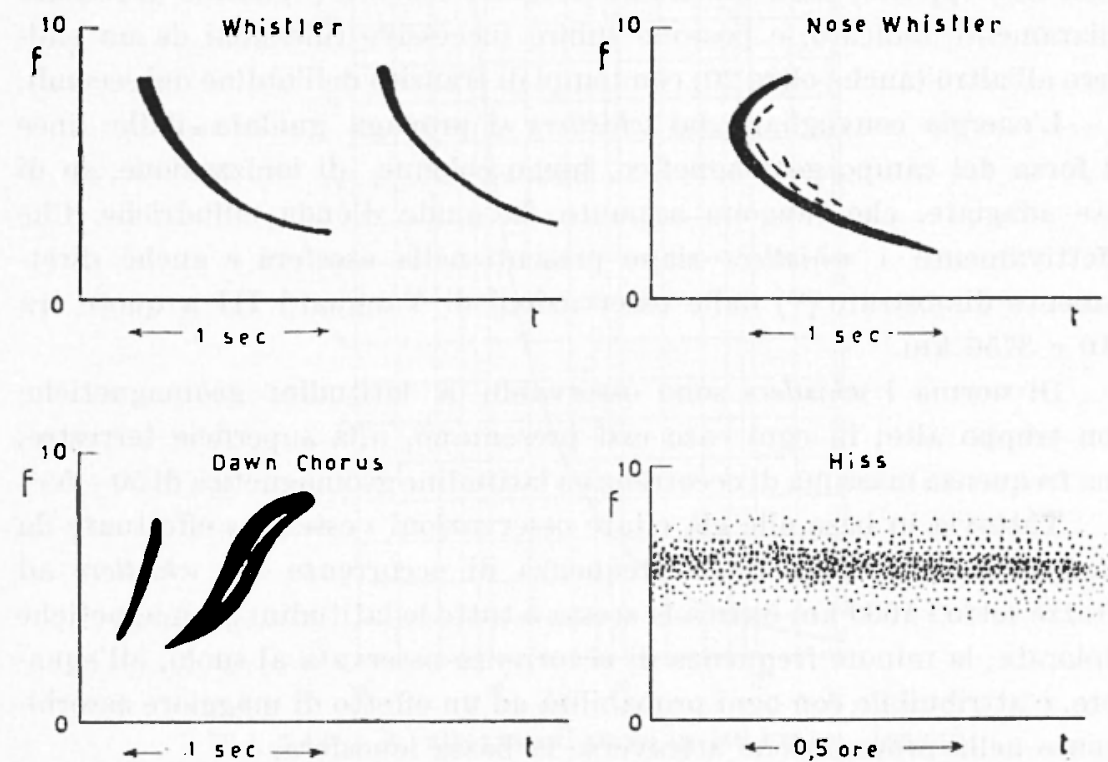

Fig. 5.10 - Andamento temporale caratteristico della frequenza per vari tipi di emissione elettromagnetica di frequenza acustica: $f$, frequenza in $\mathrm{kHz}$; sono indicate le durate tipiche.

solto i nomi di whistlers (fischi), dawn chorus e hiss, con ulteriori più particolari specificazioni a seconda delle loro caratteristiche. Si tratta di onde elettromagnetiche di frequenza acustica o nltracustica che si propagano nella esosfera lungo percorsi praticamente coincidenti con le linee di forza del campo geomagnetico. La classificazione nelle tre dette categorie a stabilita in base alla variazione temporale della frequenza e alla clumata di ciascun evento (r. fig. j.10): in particolane mentre whistlers e danen chorus durano per tempi dell'ordine del secondo o della frazione 
di secondo e variano mpidamente di frequenza, l'hiss puo invece durare fino a qualche ora con emissione sensibilmente stazionaria in una banda di frequenze ampia qualche $\mathrm{kHz}$.

5.2.1. Whistlers. - Sebbene la scoperta del fenomeno $\left({ }^{38}\right)$ risalga al 1919, si deve a Storey ${ }^{39}$ ) un esame approfondito dei fatti sperimentali e una classificazione dei whistlews in tipi, nonché uno schema di interpretazione del fenomeno. Questo risulta chiaramente associato ai disturbi atmosferici originati da seariche elettriche nella troposfera; i brevi impulsi elettromagnetici che li costituiscono (10) possono facilmente propagarsi dal punto di origine al punto geomagneticamente coniugato nell'emisfero opposto, come misure di Morgan e Allcock (11) hanno di recente chiaramente indicato, e possono subire suceessive riflessioni da un emisfero all'altro (anche oltre 20 ) con tempi di transito dell'ordine dei secondi.

I'energia convogliata dai whistlers si propaga guidata dalle linee di forza del eampo geomagnetico, lungo colonne di ionizzazione, su di esse adagiate, che fungono appunto da guide d'onda cilindriche. Che effettivamente i rhistlers siano presenti nella esosfera è anche direttamente dimostrato ${ }^{(2)}$ dalle osservazioni di Vanguard III a quote tra 510 e $3750 \mathrm{~km}$.

Di norma i whistlers sono osservabili a latiturlini geomagnetiche non troppo alte; in ogni caso essi presentano, alla superficie terrestre, una frequenza massima di occorrenza a latitudine geomagnetica di 50 $\div 55^{\circ}$.

Tuttavia in base alle già citate osservazioni " esterne " effettuate da Vanguard III risulta che la frequenza di occolrenza dei whistlers ad altezze sot to i $1000 \mathrm{~km}$ de circa la stessa a tutte le latitudini geomagnetiche esplorate; la minore frequenza di occorrenza osservata al snolo, allequatore, è attribuibile con ogni probabilità ad un effetto di maggiore assorbimento nella propagazione attraverso la bassa ionosfera.

In qualche caso sono stati osservati whistlers anche a latitudini molto alte: in particolare, Martin $\left({ }^{13}, 11\right)$ ha osservato a Scott Base, a poco più di $10^{\circ}$ dal polo geomagnetico sud, treni di echi distanziati di circa 2,5 secondi.

Facendo riferimento alla fig. 5.7, si vede che se i punti coniugati corrispondono a una latitudine geomagnetica di $50^{\circ}$ la massima distanza geocentrica della guida d'onda, ovviamente in comispondenza del piano equatoriale, ì di circa 2,5 raggi terrestri. A latitudini geomagnetiche intorno a $80^{\circ}$ la corrispondente distanza delle linee di forza magnetiche sarebbe di circa 25 raggi terrestri: tutto ciò che si è detto nei precedenti paragrafi sull'andamento del campo magnetico terrestre permette 
senz'altro di escludere che in questo caso i whistlers osservati a Scott Base si propaghino lungo le linee del ampo magnetion si deve ritenere che condizioni ionosferiche favorevoli ne possano talvolta consentire la propagazione da latitudini più basse.

Appare chiaro quindi che lo studio dei whistlers consente di ottenere informazioni sulla distribuzione di densita ionica a quote notevolmente superiori a quelle ionosferiche. Ciò in particolare perché il tempo di transito da un emisfero all'altro dipende in modo piuttosto semplice dalla distribuzione di densità elettronica lungo il pereorso dell'onda.

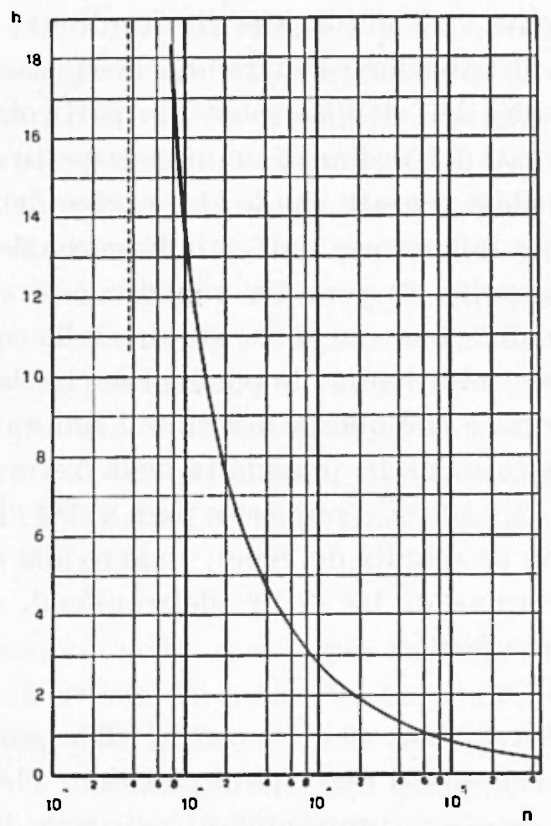

Fig. $5.11-h$, altezza sul stolo in $10^{3} \mathrm{~km} ; n$, densita elettronica in $\mathrm{em}^{-3}$ (secondo Schmelovskș).

Già dalle citate ricerche di Storey risultano, alle massime distanze da Terra, densita elettroniche assai elevate, dell'ordine delle centinaia di elettroni/(-m². Valori cosi alti mostrano che al di sopra del massimo di densità elettroniea della regione $\mathrm{F}$ la diminuzione di densità è assai lenta e comunque di tipo non esponemziale. Combinando risultati ot tenuti dallo studio delleffetto Faraday dei segnali emessi da Sputnik III con quelli desunti dall'esame dei whistlers, Schmelovsky (25) ha costruito un modello di distribuzione di densità elettronica con la distanza geocentrica che riportiano in fig. 5.11. 
C'è da aggiungere che sussiste una correlazione positiva tra frequenza reritica dello strato F'2 e riturdo tra echi successivi $\left({ }^{46}\right)$; Allcock e MIorgan (17) hamno trovato inoltre ma correlazione tra il ritardo con cui i whistlers prodotti da una ben identificata searicar elettrica tornano, dopo la prima riflessione nel punto coniugato, nel punto di origine e il numero di Wolf: l'andamento mese per mese della media mensile dei ritardi appare sensibilmente parallelo a quello dei numeri di Wolf osservati da uno a due mesi prima; il coefficiente di correlazione laggiunge in questo caso un valore di circal $+0,8$.

Ricordiamo, come si è detto, che il ritardo tra echi sucecessivi è condizionato dalla distribuzione elettronica nella esosfera e che taluni parametri caratteristici dell'attività solare (in particolare l'attività coronale) appaiono ritardati dell'ordine di un mese rispetto ai numeri di Wolf; sembra quindi plausibile pensale che lo stato fisico della esosfera sia più o meno sensibilmente influenzato dall'attività coronale. Un tal modo di vedere è, per es., suggerito $\left.{ }^{48}\right)$ per altra via dalle osservazioni della madioemissione nel campo delle onde metriche emesse dalla corona solare. Chap)man ${ }^{(9,50}$ ) suggerisce addirittura la possibilità che la rorona solatre si estenda fmo alla Terra e che questa deseriva il suo moto di rotazione in essa immersa; il gas coronale in prossimità della Terra arrebbe temperatura dell'ordine di $2 \cdot 10^{5}$ oK (supposta pari a $10^{6}$ oK la temperatura della corona solare in prossimità del Sole); quanto alla densità elettronica essa si valuta dell'ordine di $10^{2} \div 10^{3}$ elettroni/cms appunto quale risulta dallo studio dei whistlers.

5.2.2. Dawn chorus, hiss. - I Di contro all'origine atmosferica dei whistlers, si ritiene che questi due tipi di emissione elettromagnetica abbiano origine nella esosfera, propagandosi poi verso la bassa atmosfera lungo le linee del campo magnetico terrestee, con modalità analoghe a quelle dei whistlers. Fon è ancora chiaro se anche daun chorus e hiss possano propagarsi tra punti coniugati da un emisfero all'altro: mentre Helliwell $\left({ }^{51}\right)$ lo esclude, Alleock $\left(^{52}\right)$ a lillis $\left({ }^{53}\right)$, invece, ritengono possibile tale propagazione per il daun chorus e, rispettivamente, per i più intensi hiss.

Anche daun chorus e hiss si osservano più frequentemente a latitudini geomagnetiche non troppo alte $\left(50 \div 60^{\circ}\right)$.

Sono in corso di studio da parecchi antori le variazioni diurne e stagionali nonchè la correlazione tra occorrenza di whistlers, dawn chorus e hiss e attività geomagnetica e solare. I risultati sono ben lontani dall'essere conclusivi. Si può dire che dawn chorus e hiss mostrano una certa 
associazione con le perturbazioni magnetiche a dei raggei cosmici, nel colso delle quali appaiono con maggiore frequenza che in periodi calmi. In generale sembra provato $\left.{ }^{5,4}, 55\right)$ che la frequenza di occorrenza ò massima in colrispondenza alla fase principale delle tempeste magnetiche, il che puó far pensale a un collegamento della emissione con la corrente amulare equatoriale cui si tende ad attribuire la fase principale della perturbazione magnetica.

L'osservazione che le lines di forza magnetiche uscenti datle zone di massima frequenza attraversano il piano equatoriale geomagnetico a distanze di $3 \div 4$ ragegi terestri induce a pensare che dawn chorus e hiss possano avere connessione con i processi fisici che arvengono nelle fasce di radiazione di van Allen. Vari neceanismi di origine e di amplifeazione della potenza trasmessa sono stati proposti da diversi antori, nel comume presupposto che l'energia trasmessa dalleonda sia oftemuta a spese dell'energia cinctica di particelle cariche penetranti nol campo magnetico terrestre: Gallet e Helliwell $\left(^{56}\right)$ hamno suggerito che l'onda elettromagnetica si generi per interazione tra le particelle che originano il rumore termico normalmente presente nella esosfera e fasci di protoni veloci; Mac. Arthur $\left({ }^{57}\right)$ ha pensato a radiazione di sinceotrone emessa dai protoni spilalizzanti intorno al ampo magnetico terrestre; Ellis $\left({ }^{58}\right)$ hal suggerito invere trattarsi di radiazione Cerenkor emessa nella penetrazione di protoni nella esosfera ionizzata.

5.2.3. Informazioni nello spazio jùu prossimo alla Terra possono ottenersi anche dallo studio dei radioechi dalla luna: in particolare, dalle caratteristiche della polarizzazione delle radioonde si deduce $\left.{ }^{59}\right)$ rhe complessivamente il mumero di elettroni presenti a quote superiori a quella del massimo di densità dello stento Fo è varie volte maggiore del numero di elettroni presenti a quote inferiori e presenta rispetto a questo variazioni sia diurne che stagionali più regolari. A distanze maggoiori si hanno indicazioni assai incerte: subordinatamente alle notevoli incertezze del metodo, Blackwell $\left({ }^{60}\right)$, dallo studio della polarizzazione della luce zodiacale, ritiene che a distanze geocentriche dellordine dellunità astronomica la densità elettronica debba essere praticamente nulla.

\subsection{Le fasce di radiazione di van Allen.}

Una delle più importanti scoperte compinte negli nltimi anni ̀̀ stata quella della esistenza di una estesa regione circostante la Terra 
nella (quale sono ac(umulate (" intrappolate») in vari strati, in gran numero, particelle cariche, di energia relativamente alta (dellordine dei keV e fino a qualche decina di MeV) ma tuttavia molto piccola rispetto a quella media della radiazione cosmion primaria. Si tratta delle cosidette fasce o cinture di radiazione di van Allen. La presenza e le caratteristiche di tale regione di accumulo appaiono strettamente condizionate dal campo magnetico terrestre.

5.3.1. Le prime segnalazioni dell'esistenza delle fasce di van Allen sono dovute appunto a van Allen e coll. (61, 62) i quali, nell'intento di misurare la radiazione cosmica fuori dell'atmosfera, rilevarono con contatori G.M. a bordo dei satelliti Explorer I (1958 a) ed Explorer III (1958 $\gamma)$, che al crescere della quota l'intensità misurata cresceva lentamente fino a cirea $700 \mathrm{~km}$, nel rapporto previsto tenendo conto della progressiva apertura dei coni permessi di Störmer e della diminuzione dell'angolo solido sotteso dalla Terra, e che, a quote oltre i $1000 \mathrm{~km}$, l'intensità prendeva invece a crescere molto rapidamente in modo del tutto imprevisto ma, commune, dipendente da latitudine e longitudine, finché a quote sopra i $2000 \mathrm{~km}$, i contatori cessavano di contare. L'interpretazione data a queste osservazioni fu che a quote oltre i $2000 \mathrm{~km}$, l'intensità di particelle dovesse essere così elevata da "bloccare " i contatori: almeno 1700 particelle $\cdot$ cm $^{-2} \cdot$ sec $^{-1}$ nell'ipotesi che si trattasse di particelle penetranti e addirittura $10^{4}\left(\mathrm{Cm}^{-2} \cdot\right.$ sec- $^{-1}$ nell'eventualita che si trattasse di elettroni poco energetici dei quali si misurava la bremsstrahlung.

Da notare che gli strumenti portati da Sputnik II $(1957 \beta)$ avevano indicato ( $\left.{ }^{63}\right)$ un ammento massimo di intensità di solo un fattore 2, di breve durata, che fu attribuito a uu burst di extraparticelle. E ora chiaro che la differenza nei risultati ottenuti da van Allen e coll. e da Vernov e coll. è dovuta alle diversità di traiettoria e di apogeo dei satelliti (più basso quello degli Sputnik).

5.3.2. Configurazione delle cinture. - Ricerche sistematiche sulle cinture di radiazione furono iniziate con i lanci di Sputnik III (64. 65) e di Explorer IV $(1958 \varepsilon)\left({ }^{66}\right)$, i eui strumenti, in particolare scintillatori, erano atti a misurare intensità elevatissime. La fig. 5.12 a riporta come esempio una mappa delle linee di uguale intensità osservate nell'intervallo di longitudini geografiche $280^{\circ} \pm 20^{\circ}$ est da uno dei rivelatori portati da Explorer IV. Dal confronto tra gli andamenti delle intensità per diverse bande di longitudini e in periodi diversi di osservazione, van Allen e coll. 
deducevano uno stretto controllo geonagnetico delle intensità misurate, le quali presentavano una distribuzione sensibilmente simmetrical rispetto alla latitudine geomagnetical (e non rispetto alla latitudine geografica).
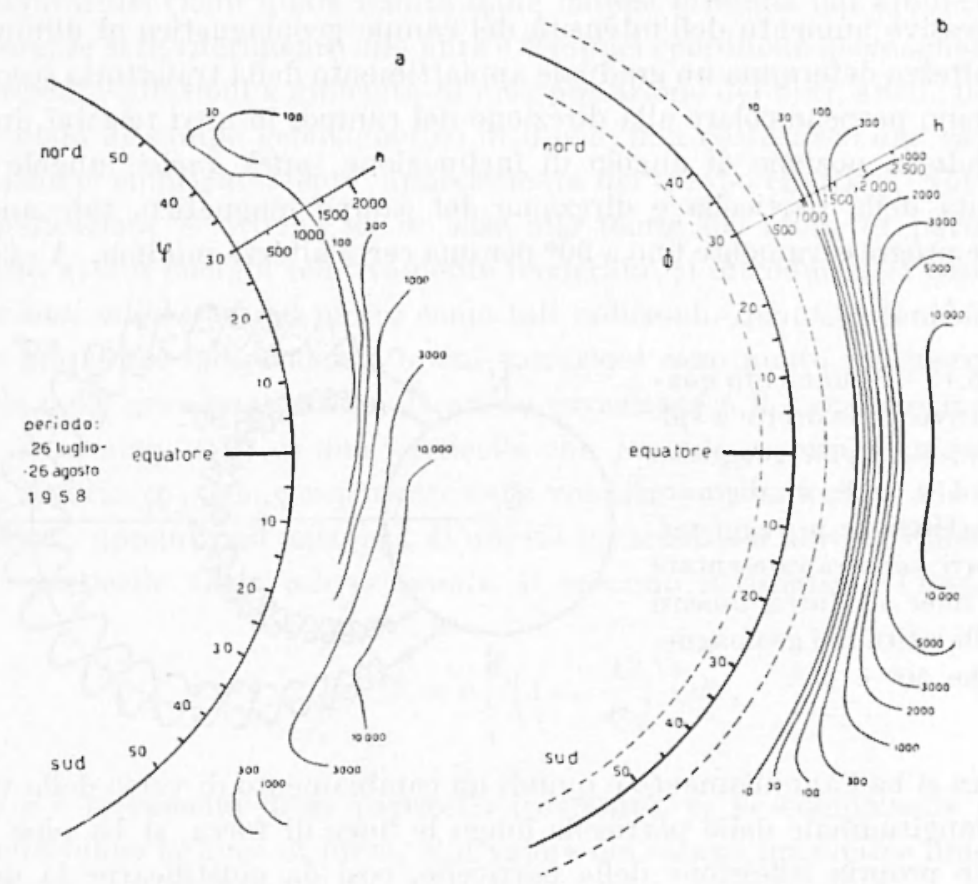

Fig. 5.12 - In fig. $a$, linee di uguale intensità di conteggio (impulsi/ sec) misurate da un contatore G.M.; $h$, altezza in $\mathrm{km} ; \varphi$, latitudine reografica (secondo van Allen. Illlwain e Lurwig): in fig. b) linee di ugruale intensita di contegrgio di Explorer IV, nel sistema di coordinate polari $R, \Phi$ equivalente al sistema di coordinate $B, L$, definite da Ircllwain. Le linee trattergiate indicano approssimativamente le escursioni massime della superficie terre. stre effettiva nelle coordinate polari (secondo McIlwain).

Con la particolare disposizione dei rivelatori fu anche possibile stabilire una interessante anisotropia della radiazione di van Nllen: questa presentava intensità maggiore in direzioni sensibilnente oblique rispetto alle linee di forza del campo geomagnetico, cosi da dar luogo ad una distribuzione angolare più o meno appiattita, "a disco ", a seconda della latitudine geomagnetica e della altezza sul suolo.

Secondo la prima interpretazione della fenomenologia, interpretazione che si è successivamente rivelata colretta, laumento di intensita is 
dovulo a particelle carche, presumibilmente elettroni e protoni, intrappolate su orbite periodiche avolgentisi in forma di spirale intorno alle linee di forza del campo magnetico terrestre (fig. 5.13). In tale schema, il progressivo aumento dell'intensita del campo geomagnetico al diminuire dellaltezza determina un graduale appiattimento della traiettoria secondo un piano perpendicolare alla direzione del campo; in altri termini, introducendo la nozione di angolo di inclinazione (pitch angle), angolo tra relocità deela particella e direzione del campo magnetico, tale angolo ressee progressivanente tino a $90^{\circ}$ per ma certa altezza minima. A questa

Fig. 5.13 - Andamento qua litativo delle orbite a spi. rale: $X$, s poli greografici nord a sud; $r$, distunze geocentric:le in ragrgi terrestri: sono rappresentate le linee di forza uscenti dalle latitudini geomagne tiche $500^{\circ}+60^{\circ}$.

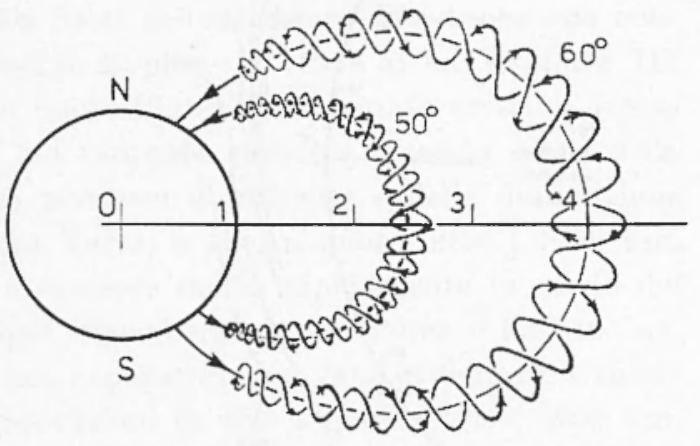

altezza si ha l'ammullamento e quindi un cambiamento di verso della relocità longiturlinale delle particelle lungo le linee di forza, si ha rioè ma vera e propria riflessione delle particelle, cosi da giustificame la denominazione di punto di riflessione (mivor point). Si noti che la densita di materia che le particelle incontrano nel loro moto da un punto di riflessione allablto, situato a latitudine geomagnetica uguale ex opposta, è massima appunto in tali due punti per cui è sopratutto nei punti di riflessione che le particelle stesse possono sf uggere dalle fasce di ladiazione per interazioni di varia natura con le particelle costituenti l'atmosfera.

Una confema della situazione fisica sopra indicata e più in particolare informazioni sulla distribuzione radiale e quindi sulla estensione della zona di ratiazione intorno alla Terra sono dorute alle suceessive misure con sonde sparziali (Pioneser I $\left({ }^{67}\right)$, Pionerer III $\left({ }^{63}\right)$, Lamik I e

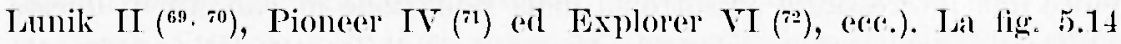
mostra i risultati di alcume delle misumzioni. Si reve ormai ritenere provata l'esistenza di due zone a simmetria cireolare intorno allasse del dipolo magnetico terrestre (fig. 5.15), ma più interma con centro a uma distanza geocentrica di circa 1,6 raggeri terrestri (circa $3600 \mathrm{~km}$ dal suolo)

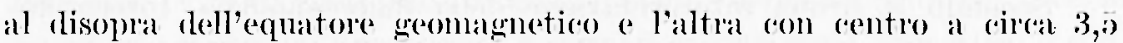
raggeri terrestri (cirea $16.000 \mathrm{~km}$ dal suolo). 
5.3.2.1. Il sistema di coordinate "naturali" di McIlarain. - Isal grande quantita di misure e la loro notevole diversita in punti anche relativamente vieini quale risulta dalle mappe ottenute dai satelliti puó portare, se si fa riferimento alle pure esemplici coordinate geomagnetiche, a notevoli confusioni e difficoltà di interpretazione dej dati, anche jerché il modello di ampo geomagnetico di dipolo non costituisce ma rappresentazione sufficientemente approssimata del campo eflettivo. Soterole semplificazione si oftiene se, in base alla teoria del moto di particelle cariche arenti energia relativamente moderata, si introducono i cosidetti invarianti adiabatici del moto, come tali indicando quantita funzione di altre grandezze indipendenti, le cui variazioni sono molto più piecole di quelle delle grandezze stesse. Il primo invariante i il momento magnetico $\mu=m e^{2} \perp /(2 B)$ di una particella che procede a spirale lungo una linea di forza $\left(v_{\perp}\right.$ è la componente della velocita perpendicolare alla linea di forza); appunto all'esistenza di questo invariante si deve la riflessione delle particelle tra i mimor points. Il serondo invariante à lintegrale

$$
I=\int_{r_{1}}^{r_{2}} v_{\|} d l=v \int_{r_{1}}^{r_{2}}\left(1-\frac{B}{B_{r}}\right)^{1 / 2} d l,
$$

dove $v$ e la velocità della particella (costante), $v_{\|}$la componente della velocita lungo la linea di forza, $B$ il valore del ampo magnetico lungo la linea di forza, $B_{r}$ il suo valore nei due punti di riflessicne $r_{1}$ e $r_{2}$. Come conseguenza dell'esistenza di questo invariante, le particelle in tutto il loro moto (r. anche 5.3.5) rinangono sempre su quelle linee di forza per cui il valore di $I$ ì costante; si viene quindi formare un "guscio" (shell) di particelle, modellato dallandamento delle lirwe di forza, guscio che deve rimanere pressoché invariato nel tempo. L'esistenza di questo secondo invariante ha dato a MeIlwain ( $\left.{ }^{73}\right)$ la possibilitì di definire un sistema " naturale " di due coordinate, costituito dal valore sealare del campo magnetico $B$ a dall’invariante adiabatico longitudinale $I$ o, in sostituzione di questo e più vantaggiosamente, da una lunghezza $L$, funzione di $B$ e di $I$, il eui significato fisico, nel caso particolare di campo di dipolo, è la distanza geocentrica dela intersezione tra linea di forza e piano equatoriale geomagnetico. Eे ben evidente che uno dei principali vantaggi del nuovo sistema di coordinate a quello di ridure le coordinate necessarice a due, $B$ e $L(0, I$ ) in luogo delle tre, altezza, latituline e longitudine geomagnetiche. Un sistema equivalente, ma che si presta meglio ad una rappresentazione intuitiva, è costituito dalle due coordi- 
nate $R$ e $\Phi$, che rappresentano rispettivamente una distanza geocentrica e una latitudine geomagnetica, per altro fittizie, che si ottengono dalle $B$ a $L$ applicando ad esse le relazioni del dipolo:

$$
B=\frac{M}{R^{3}}\left(4-\frac{3 R}{L}\right)^{1 / 2}, \quad R=L \cos ^{2} \Phi .
$$

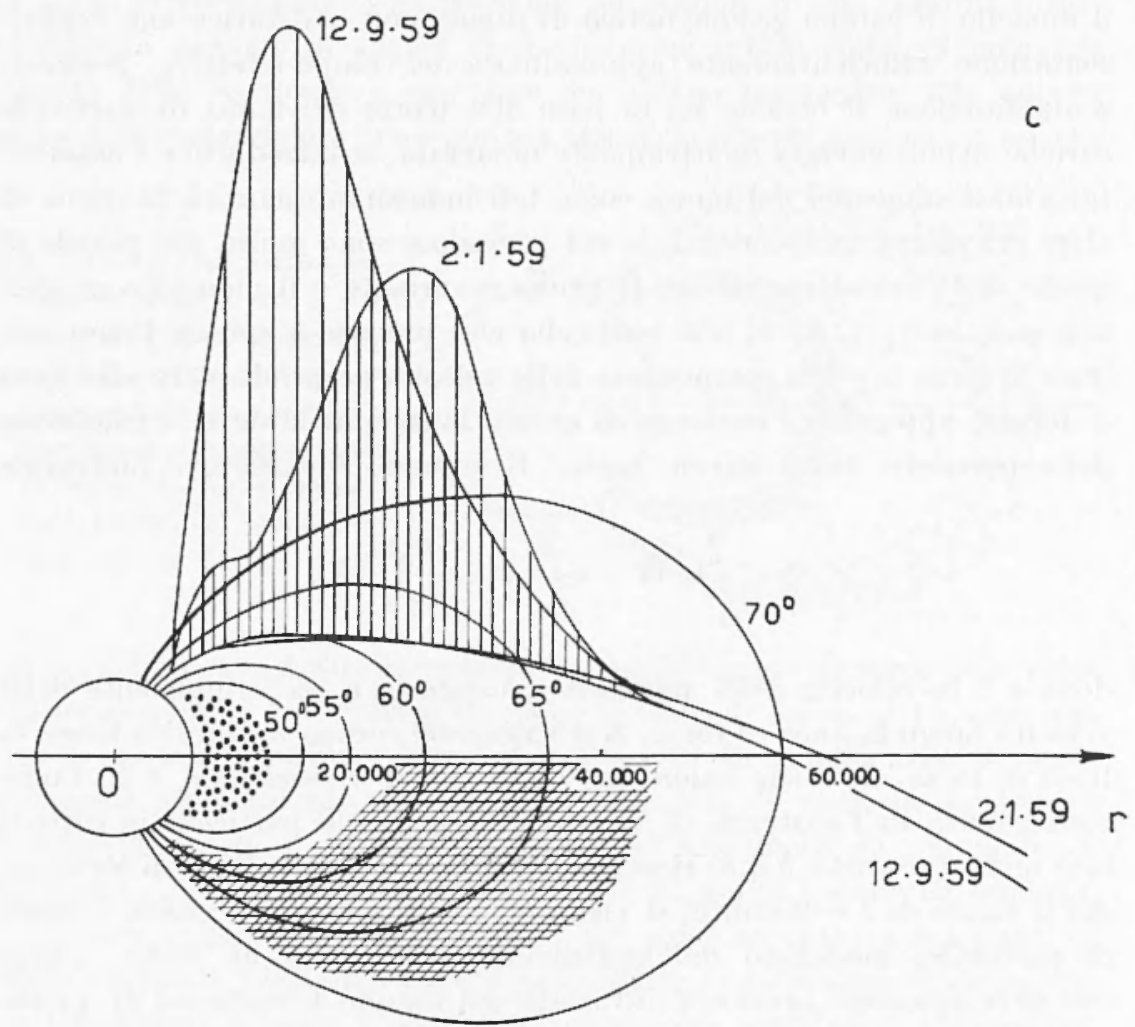

Fig. 5.14 - a, intensità (in impulsi/sec) determinata da Pioneer III e Pioneer IV; i due rani $\alpha$ e $\beta$ rappresentano due andanenti che sono entrambi compatibili, entro grli errori di misura, con il contegrgio apparente (secondo van Allen e Frank); $b$, intensità della radiazione ionizzante (in roentgen/ora) misurata da Pioneer I (secondo Rosen, Sonett. Coleman e IICllwain); c. traiettorie di Lunik I e Lunik 11, e su ciascuna di esse, trattegrerata verticalmente, intensità di contegrio (secondo Vernov. Chudakov, Vakulov, Logachev e Nikolaev). In tutti i grafici $r$ è la distanza geocentrica in $\mathrm{km}$.

Nelle nuove coordinate, i possibile una migliore organizzazione (fig. 5.12 b) dei dati sperimentali rispetto a quella possibile in coordinate 


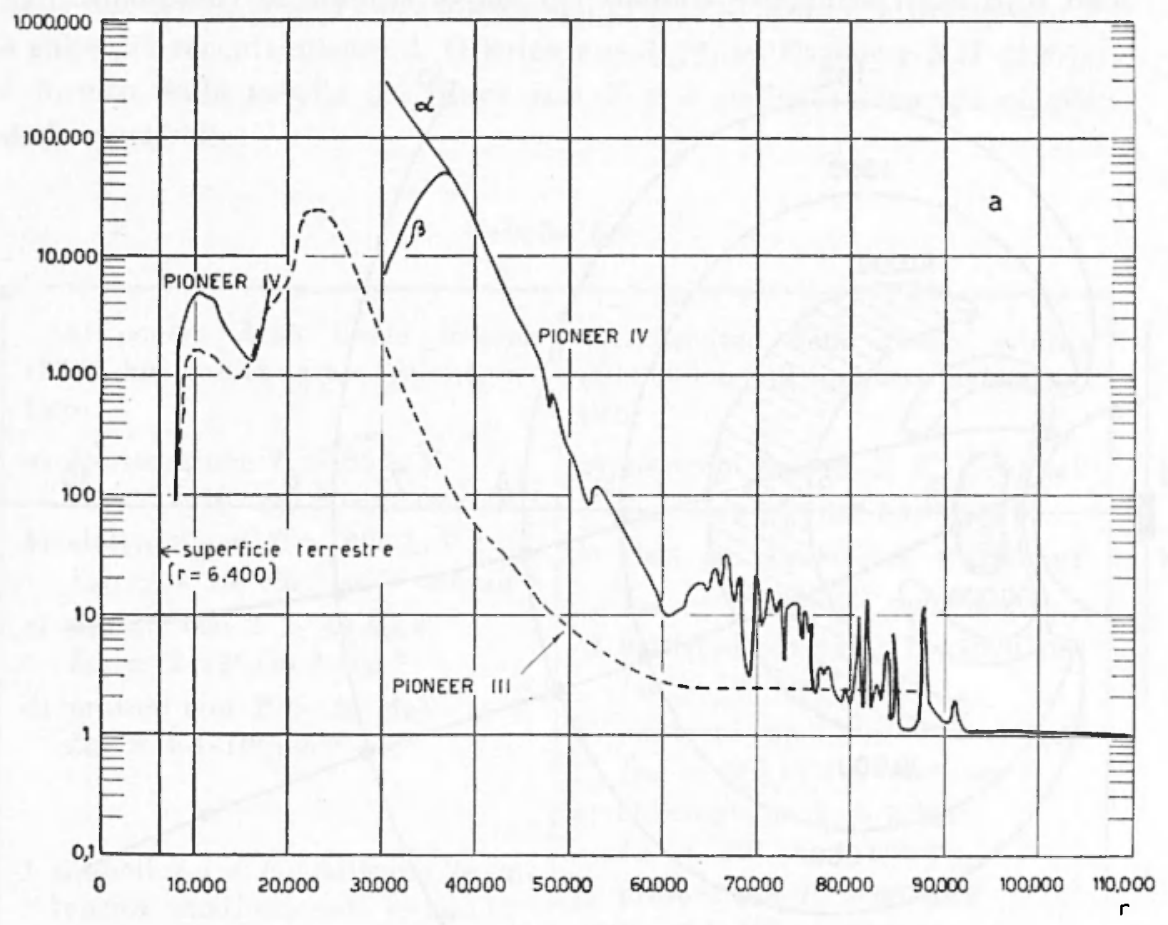

Fig. $5.1+a$

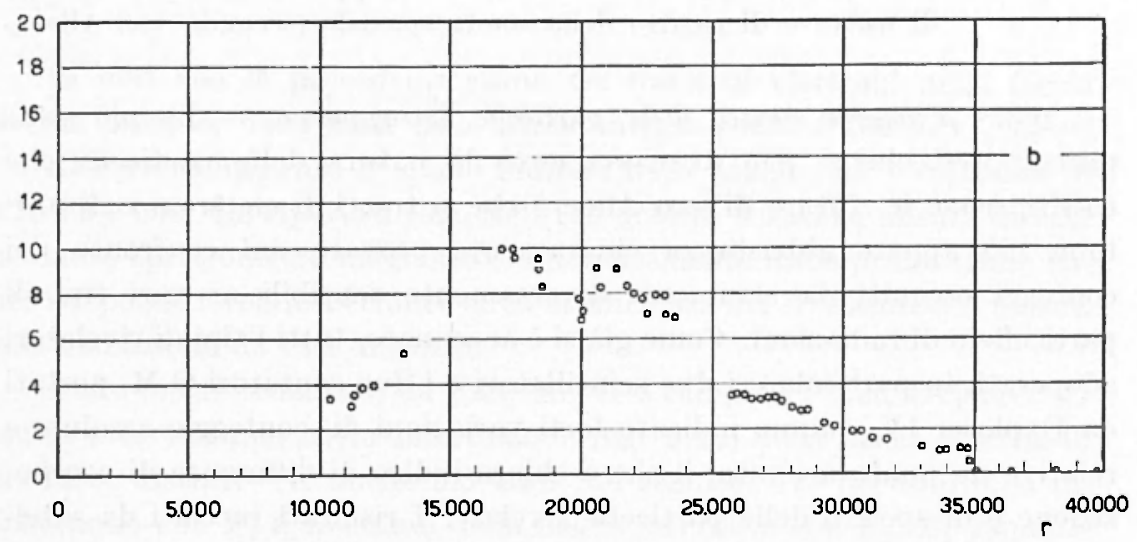

Fig. $5.14 b$ 
geomagnetiche mostrata in fig. 5.12 a. Aleuni dei risultati più recenti sono stati assai utilmente studiati nel nuovo sistema di coordinate.

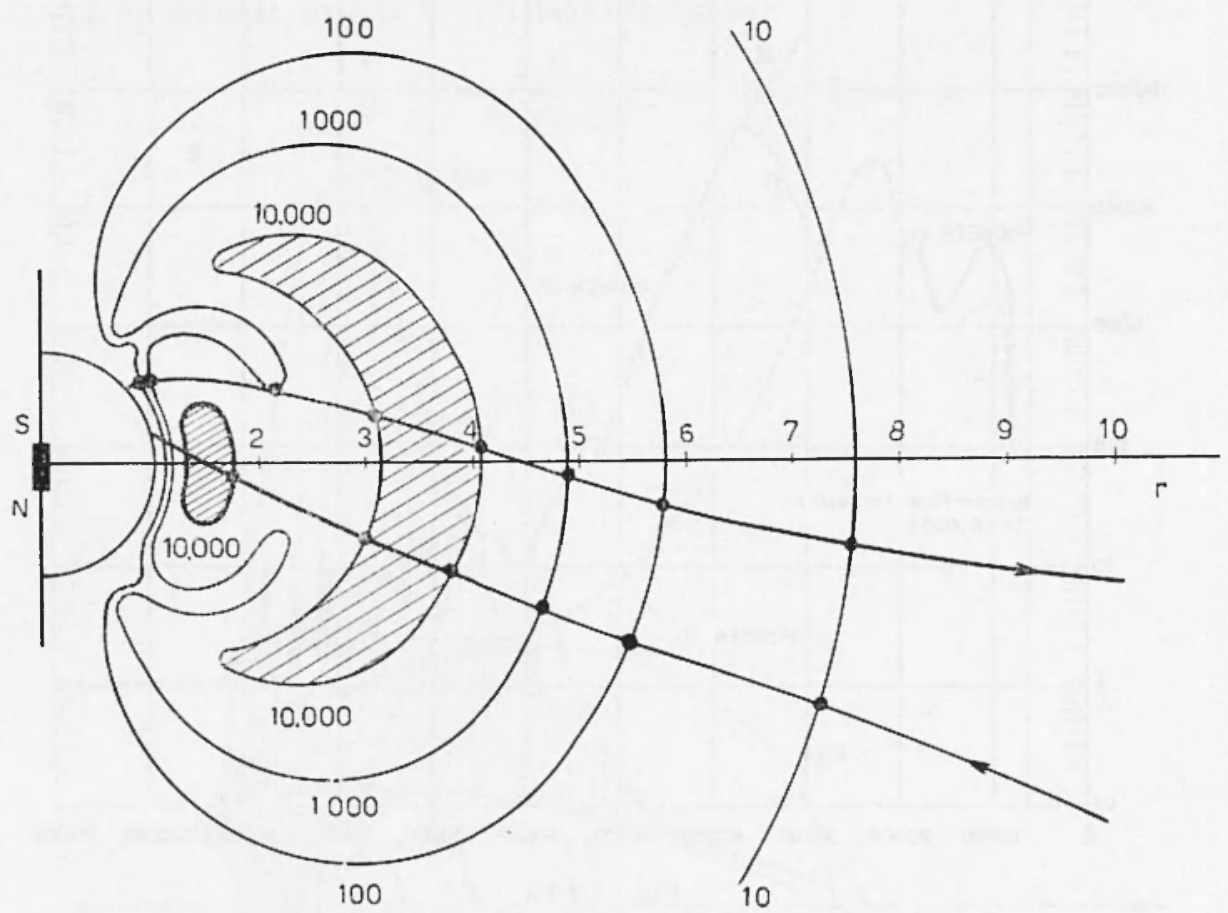

Fig. 5.15 - Intensità di contegroo (in impulsi/sec) misurata da Pioneer I: $r$, distanza geocentrica in raggi terrestri; $\mathrm{S}, \mathrm{X}$, poli sud e nord del dipolo magnetico terrestre: somo rappresentate le traiettorie li uscita e di rientro della sonda spaziale (secondo van Allen).

5.3.3. Flusso e spettio delle particelle intrappolate. — Ia più semplice ipotesi che si puo avanzare circa la natura delle particenle che costituiscono le cinture di van Allen è che si tratti di elettroni e di protoni; ciò appare abbastanza chiaramente provato dal confronto dei conteggi eseguiti da strumenti diversmmente sensibili ai vari tipi di particelle o di radinzioni. Come già si à accemnato, tutti i tipi di rivelatori adoperati, in particolare i due seintillatoni e i due contatori G.M. portati da Explorer IV, hamno indicato forti variazioni di conteggio assolute e relative da punto a punto, il che è chiaro indice di differenze di composizione e di spettro delle particelle rivelate. I risultati mecolti da satelliti a sonde spaziali hamo ormai permesso di stabilire con certezza che la fascia interna è costituita da radiazione più dura di quella che costituisce 
la fascia estema. Un quadro indicativo delle intensità, o meglio dei flussi. nelle due fasce, basato sulle misure dei gruppi di Iowa $\left({ }^{74}\right)$, di Chicago ( $\left.{ }^{72}\right)$

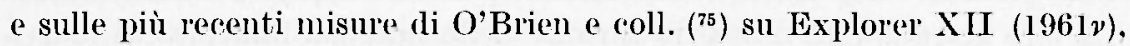
è fornito dalla tabella 5.2 , dove con $T$ si è indicata l'energia cinetica delle particelle.

\section{Tabella 5.2}

\begin{tabular}{|c|c|}
\hline $\begin{array}{l}\text { Al centro della fascia interna } \\
\text { (3600 km sull equatore geomagne- } \\
\text { tico). } \\
\text { a) elettroni con } T>20 \mathrm{keV} \\
I_{\text {un }} \simeq 2 \cdot 10^{9} \mathrm{~cm}^{-2} \cdot \mathrm{sec}^{-1} \cdot \mathrm{sterad}^{-1} \\
\text { b) elettroni con } T>600 \mathrm{keV} \\
I_{\text {un }} \simeq 1 \cdot 10^{7} \mathrm{~cm}^{-2} \cdot \mathrm{sec}^{-1} \cdot \mathrm{sterad}^{-1} \\
\text { c) protoni con } T>40 \mathrm{MeV} \\
I_{\text {on }} \simeq 2 \cdot 10^{4} \mathrm{~cm}^{-2} \cdot \mathrm{sec}^{-1} \\
\text { d) protoni con } T>75 \mathrm{MeV} \\
I_{\text {on }}=1,4 \cdot 10^{3} \mathrm{~cm}^{-2} \cdot \mathrm{sec}^{-1} \\
\text { I simboli } I_{\text {un e } I_{\text {on }} \text { indicano le in- }} \\
\text { tensità unidirezionali e ommidi- } \\
\text { rezionali. }\end{array}$ & $\begin{array}{l}\text { Al centro della fascia esterna } \\
\text { (16.000 km sull'equatore geomagne- } \\
\text { tico) } \\
\text { a) elettroni con } 45<T<60 \mathrm{keV} \\
I_{\text {on }}=\left(9_{-16}^{+16}\right) \cdot 10^{7} \mathrm{~cm}^{-2} \cdot \mathrm{sec}^{-1} \\
\text { b) elettroni con } 80<T<110 \mathrm{keV} \\
I_{\text {on }}=\left(8_{-5}^{+16}\right) \cdot 10^{7} \mathrm{~cm}^{-2} \cdot \mathrm{sec}^{-1} \\
\text { c) elettroni con } 110<T<1600 \mathrm{keV} \\
I_{\text {on }}<10^{8} \mathrm{~cm}^{-2} \cdot \mathrm{sec}^{-1} \\
\text { d) elettroni con } 1,6<T<0 \mathrm{MeV} \\
I_{\text {on }}=(2 \pm 1) \cdot 10^{5} \mathrm{~cm}^{-2} \cdot \mathrm{sec}^{-1} \\
\text { e) elettroni con } T>5 \mathrm{MeV} \\
I_{\text {on }}<10^{3} \mathrm{~cm}^{-2} \cdot \mathrm{sec}^{-1} \\
\text { b) protoni con } T>60 \mathrm{MeV} \\
I_{\text {on }} \leqslant 1 \cdot 10^{2} \mathrm{~cm}^{-2} \cdot \mathrm{sec}^{-1} \\
\text { g) protoni con } T<30 \mathrm{MeV} \text { nessuna } \\
\text { informazione signifieativa. }\end{array}$ \\
\hline
\end{tabular}

Si noti che le precedenti stime dei flussi di elettroni nella fascia esterna davano, nella zona delle basse energie (sotto i $100 \mathrm{keV}$ ), valori 100-1000 rolte maggiori di quelli indicati nella tabella; gli ultimi dati di O'Brien e coll. qui riportati sono sicuri in quanto basati su misura diretta mediante spettrometro magnetico, differentemente dalle prime stime basate su ipotesi rivelatesi erronee circa la efficienza dei rivelatori e la natura . dellar radiazione da essi misurata.

Dalle misure condotte da Fan, Meyer e Simpson $\left({ }^{72}\right)$ su Explorer VI

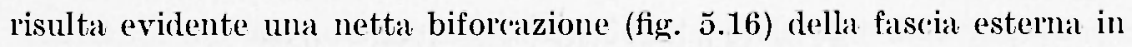
due fasce distinte (v. anche 5.3.4.2). Nel seguito, ove necessirio, si indicheranno con $P_{1}$ il massimo della fascia interna e con $E_{2}, E_{3}$ rispettivamente i due massini della fascia esterna e, più genericamente, le due regioni stesse. 
Le informazioni sulla forma dello spettro dei diversi tipi di partirelle presenti nelle cinture sono ancola piuttosto scarse e frammentarie. Ia loro attendibilità è incerta a cause delle notevoli difficoltà di individuazione della natura delle particelle rivelate.

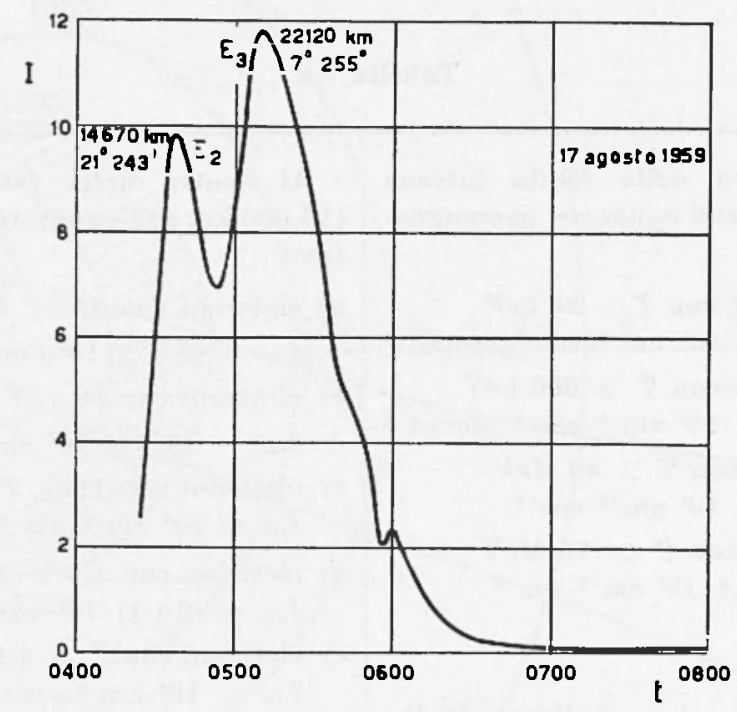

Fig. $5.16-I$, conteggio di contatore singolo in unita $10^{2}$ impulsi/see, nella fascia esterna durante il $19^{\circ}$ passaggrio; $l$, tempo medio di Greenwich: sono indicate le dintanze sul suolo dei due massimi $E_{2}, E_{3}$ e le coordinate geografiche, latitudine e longituline, alle quali essi furono riscontrati (seconto Fan, Meyer e Simpson).

5.3.3.1. Per quanto riguarda la fascia interna, e stata effettivamente possibile in vari casi una identificazione sicuma dei protoni mediante lastre nucleari inviate su razzi a suceessivamente recuperate. Ia prima misura del genere si deve a Freden e White $\left({ }^{76,}{ }^{77}\right)$, i quali (fig. 5. per energie superiori a $75 \mathrm{MeV}$ determinarono uno spettro differenziale della forma

coll

$$
d N(T)=N_{1} T^{-\gamma} d T \text { protoni } \cdot \mathrm{MeV}-1 \cdot \mathrm{cm}^{-2} \cdot \mathrm{sec}^{-1}
$$

$$
N_{1}=\left(2,6_{-1,9}^{+1,2}\right) \cdot 10^{-1}, \gamma=1,8 \pm \pm 0,08
$$

Successive misure di Armstrong e coll. $\left({ }^{78}\right)$, ancora con lastre, hammo dato no spettro simile clie presenta un massimo di intensità intorno agrli $80 \mathrm{MeV}$. 
Infine, ulteriori misure di Freden e White $\left({ }^{79}\right)$ hamno sostanzialmente confermato i loro precerlenti risultati ed esteso lo speetro fino a circa $.20 \mathrm{MeV}$, trovando un massimo intorno a to MeV.

I differenza delle misure sopla riportate che danno spettri " integiati " nel tempo e nello spazio, le misure di Nangle e Kniflen $\left(^{\circ}\right)$, ancola str lazzo, con una serie di lastre nucleari sucoessivamente esposte forniscono lo spettro in punti diversi della traiettoria (fig. 5.18 a). Allo

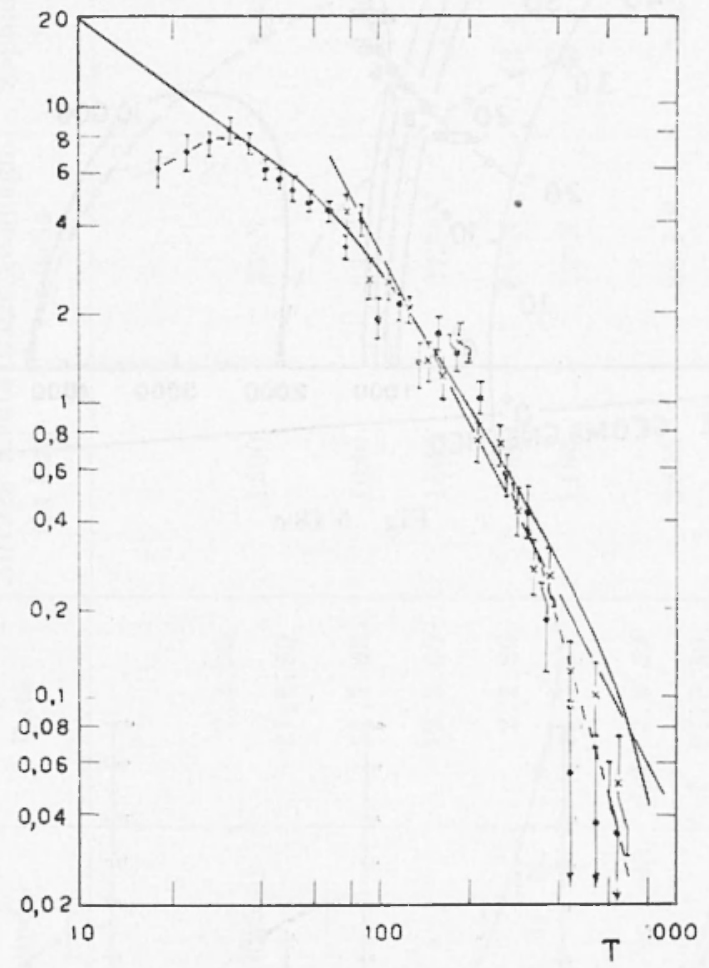

Fig. 5.17 - spettro energetico differenziale dei protoni, in protoni MeV-1 - $\mathrm{cm}^{-2} \cdot \sec ^{-1} ; T$, energia cinetica in MeV; i punti a croce e i punti pieni rappresentano rispet tivamente le misure effettuate il 7-4-195! e il 13-10-1960; la linea tratteggiata è stata calcolata con il metodo dei minimi quadrati sui punti a croce; la linea piena $\dot{e}$ nu andamento teorieo (secondo Freden e Whit().

latitudini più alte lo spettro è assai più ripido che alle latitudini minori e, comunque, più ripido di quello preverlibile in base alla teoria che attribuisce l'origine dei protoni alla disintegrazione dei neutroni di albedo dei raggi cosmici (r. 5.3.5). Le maggiori intensità di protoni nei punti 3 e 4 può essere dovuta all'iniezione dei protoni di decadimento di neutroni 


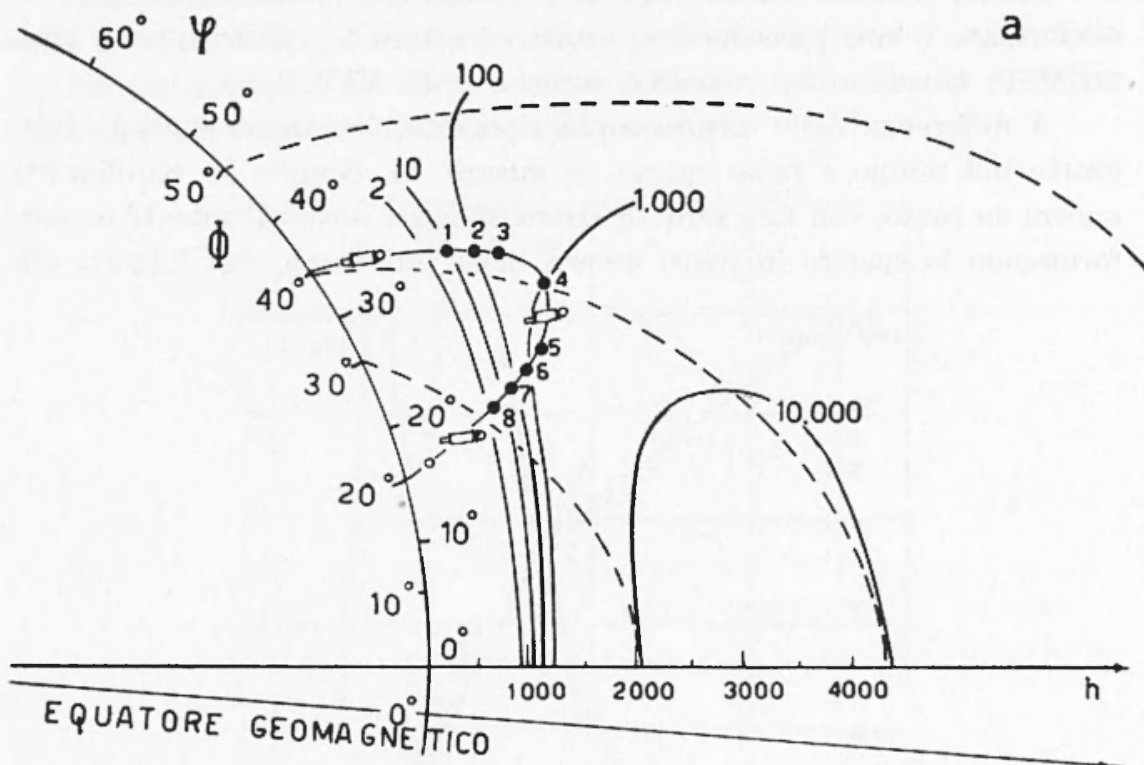

Fig. $5.18 a$
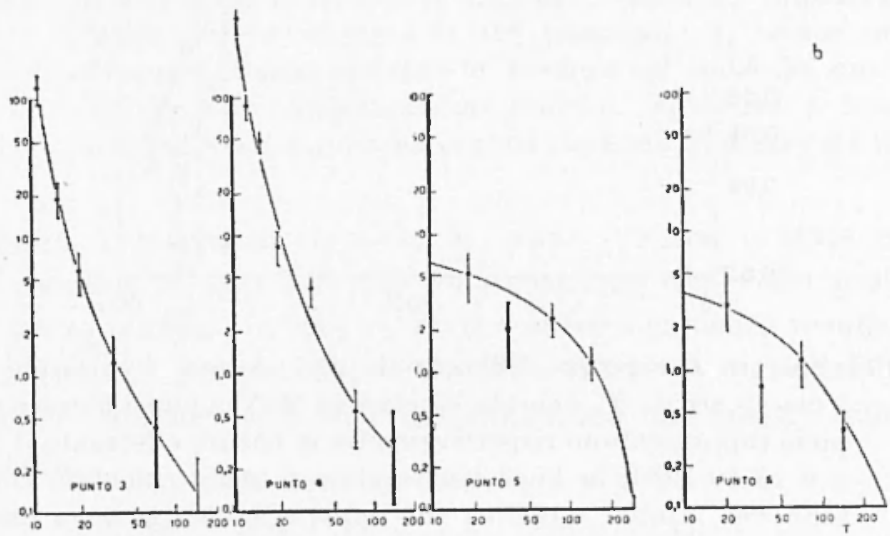

Fig. 5.18 - In fig. a traiettoria del missile; le linee tratteggiate rappresentano linee di forza magnetiche; sono anche riportate le linee di uguale intensità omnidirezionale secondo van Allen e coll.: in fig. b, spettri differenziali (in protoni $\cdot \mathrm{cm}^{-2} \cdot \mathrm{sec}^{-1} \cdot M e \mathrm{~V}^{-1} \cdot$ ste rad $^{-1}$ ) misurati in 4 diversi punti della traiettoria; $\phi$, latitudine geomagnetica, $p$, latitudine geografica: $T$, energia cinetica in MeV (secondo Naugle e Kniffen). 
'Tabella 5.3

\begin{tabular}{|c|c|c|c|c|c|c|}
\hline$A \sim \mathrm{tori}$ & Metodo & Data & $\begin{array}{l}\text { Altezza media } \\
\quad \text { (in } \mathrm{km} \text { ) }\end{array}$ & $\begin{array}{c}\text { Latit. geomagn. } \\
\text { media }\end{array}$ & $\begin{array}{c}\text { Esponente } \\
\gamma^{\prime}\end{array}$ & $\begin{array}{l}\text { Intervallo di } \\
\text { validità (MeV) }\end{array}$ \\
\hline Freden e White $\left({ }^{76,7 \overline{7}}\right)$. . & Emulsioni & $\begin{array}{r}7.4 .59 \\
21.5 .59\end{array}$ & 1200 & $25^{\circ} \mathrm{N}$ & 1,84 & $75-700$ \\
\hline Armstrong e coll. $\left(^{(\mathrm{A}}\right)$. & $"$ & 21.7 .59 & 1080 & $22^{\circ} \mathrm{N}$ & 1,80 & $80-600$ \\
\hline Naugle e Kniffen $\left(^{80}\right)$. . & $"$ & 10.9 .60 & 1600 & $27,5^{\circ} \mathrm{N}$ & 1,7 & $40-100$ \\
\hline Holly e coll. $\left({ }^{* 2}\right)$. & Cont. G. II. & $\begin{array}{r}2.2 .29 \\
21.7 .59\end{array}$ & $\begin{array}{r}980 \\
1100\end{array}$ & $\begin{array}{l}26^{\circ} \mathrm{N} \\
19^{\circ} \mathrm{N}\end{array}$ & $\begin{array}{l}1,42 \pm 0,11 \\
1,68 \pm 0,09\end{array}$ & $\begin{array}{l}>17,5 \\
>23\end{array}$ \\
\hline Hofiman e coll. $\left({ }^{81}\right)$. . & $\begin{array}{l}\text { Cont. + Cam. } \\
\text { ioniz. }\end{array}$ & $\begin{array}{l}\text { dal } 7.8 .59 \\
\text { al } 2.10 .59\end{array}$ & 2225 & $28,2^{\circ} \mathrm{S}$ & 1,65 & $>23,6$ \\
\hline
\end{tabular}


prodotti per intemazioni nucleari di protoni solari nell'alta atmosfera delle regioni polari nelle quali, come è noto (v. 3.6.4.2), è più intenso l'afflusso di protoni di energie dell'ordine delle decine o delle centinaia di MeV. A questo effetto potrebbe anche essere dovuto il massimo a qualche decina di MeV.

Altre misure con differenti rivelatori sono dovute ad Holfman; Arnoldy a Winckler $\left({ }^{81}\right)$ : essi hanno determinato mo spettro, per confronto tra le misure con contatore G.M. e camera di ionizzazione su Explorer VI, che è ancora della forma

$$
d N(T)=k T^{-\gamma^{\prime}} d T \text { protoni } \cdot \mathrm{em}^{-2} \cdot \sec ^{-1} \cdot \mathrm{MeV}-1
$$

ove $k$ varia, col punto e con il tempo, entro i limiti approssimativi $(0,9 \div 5) \cdot 10^{1}$ e $\gamma \simeq 1,65$.

L'esponente $\gamma$ dello spettro è sostanzialmente simile a quello ottenuto da Freden e White; esaminando più analiticamente i dati appare in realtà ma tendenza di $\gamma$ a crescere, assai leggermente, con la latitucline.

Infine misure con rontatori G.MI. sono state effettuate da Holly, Allen a Johnson ( $\left.{ }^{82}\right)$; i risultati, in particolare l'esponente $\gamma$ dello spettro, sono sostanzialmente simili a quelli clegli altri autori.

Fel corrispondente intervallo di energia si può ritenere che i risultati sperimentali discussi (v. tab. 5.3) siano abbastanza concordanti.

Per ciò che concerne i protoni nell'intervallo di energie sotto il MLV sono particolarmente interessanti le misure di Injun I. (1961 o $o_{2}$ ) effettuate $\left.{ }^{83}\right)$ con cristalli di CaS ad altezze di circa $1000 \mathrm{~km}$, nella fascia interna. I flussi rivelati sono molto intensi, dell'ordine di $10^{8}$ protoni . $\left(\mathrm{m}^{-2} \cdot \mathrm{sec}^{-1} \cdot\right.$ sterad $^{-1}$, nell'intervallo di energie da $1 \mathrm{keV}$ a 1 Mer; i protoni presentano la distribuzione angolare in pitch angle tipica delle particelle intrappolate. Per spiegare l'esistenza di flussi cosi elevati doruti a protoni (o eventualmente protoni e ioni) occorre supporre la presenza di una sorgente di vari ordini di grandezza più intensa di quella cui sono dovuti i protoni di energia più alta (di qualche decina di MIeV); altrimenti bisogna supporre ohe le condizioni atmosferiche che regolano la scomparsa delle particelle e quindi la loro vita media siano notevolmente diverse da quanto comunemente supposto. Si deve notare che al bordo inferiore della cintura esterma, là dove Injun I la attraversa, non è stato rivelato alcun flusso di protoni di bassa energia confrontabile con quello trovato nella cintuma interna.

Quanto agli elettroni della fascia interna, si hamno misure di Holly e Johnson ( $\left.{ }^{84}\right)$ e di Holly, Allen e Johnson $\left(^{82}\right)$. Sebbene piuttosto grosso- 
lane, le prime indicano, a $950 \mathrm{~km}$ da Terra, una prevalenza molto pronunciata di elettroni di energia sotto i 500 keV. Nelle misure più recenti, con strumentazione più complessa, in particolare atta a separare magneticamente elettroni e protoni, è stato ottenuto lo spettro integrale mostrato in figr. 5.19.

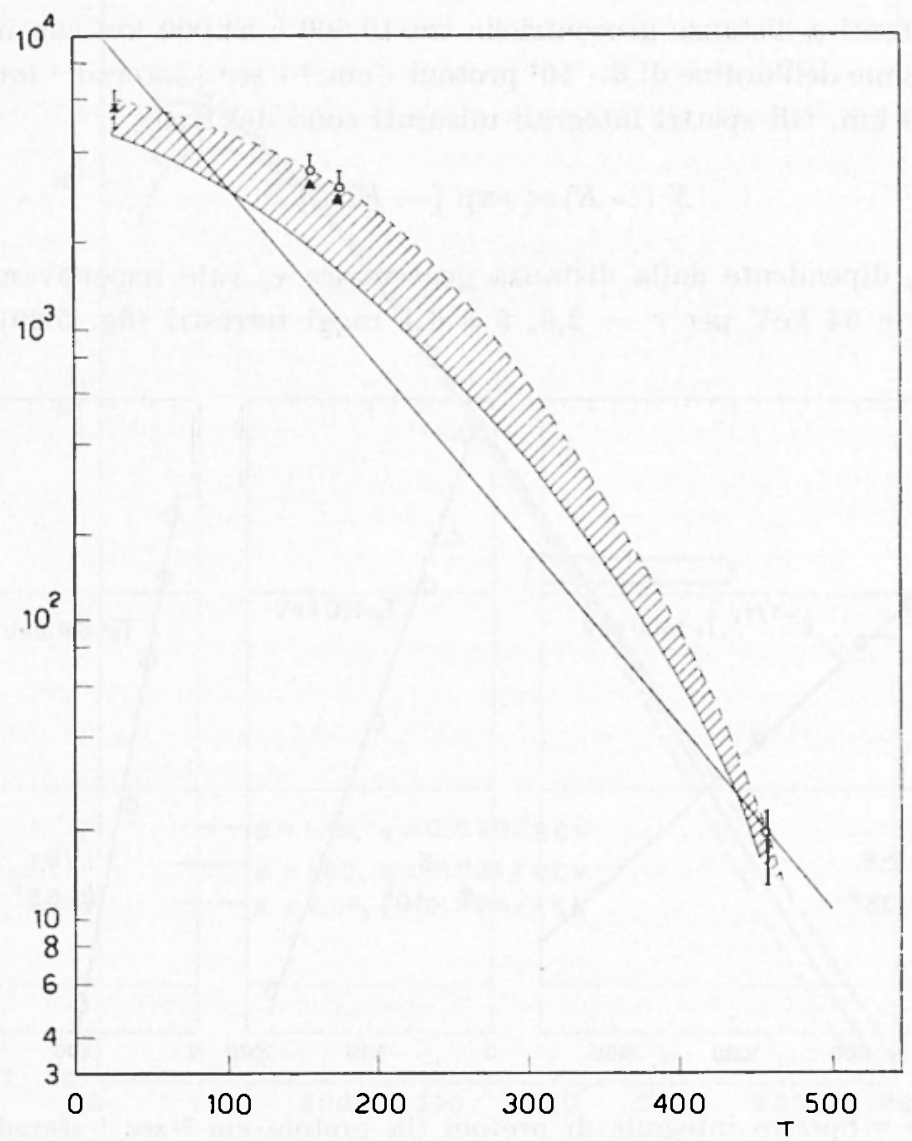

Fin. 5.19 - Spettro integrale degli elettroni della fascia interna, alla latitudine di $15^{\circ} \mathrm{N}$ e a quote tra 980 e $1100 \mathrm{~km}$, in elettroni - $\mathrm{cm}^{--} \cdot \mathrm{sec}^{-1} \cdot \operatorname{sterad}^{-1} ; T$, energia cinetica in $\mathrm{MeV}$; il tratto rettilineo riporta l'andamento trovato da Walt e coll. al bordo inferiore dolla fascia esterna (secondo IIolly, Allen e Johnson).

5.3.3.2. Una calatteristica assai rilevante della fascia di van Allen esterna è che il flusso di protoni energetici è assai più basso che nella fascia interna; ne derivano maggiori difficoltà nel determiname lo spettro: si può comunque dire con certezza che esso cade lapidamente dalla parte 
delle alte energie, nel senso che in pratica non sono presenti protoni di energia superiore a qualche decina di MeV. Dal lato delle energie più basse si hanno i dati di Davis e Williamson ( ${ }^{85}$ ) ottenuti nel periodo 16 agosto-6 dicembre 1961 con uno scintillatore portato da Explorer XII $(1961 v)$ e relative all'intervallo $0,1 \div 4,5 \mathrm{MeV}$. Protoni di tali energie sono presenti a distanze geocentriche tra 10.000 e $54.000 \mathrm{~km}$ con intensità massime dell'ordine di $6 \cdot 10^{7}$ protoni $\cdot \mathrm{cm}^{-2} \cdot \mathrm{sec}^{-1} \cdot$ sterad $^{-1}$ intorno ai $20.000 \mathrm{~km}$. Gli spettri integrali misurati sono del tipo

$$
N(>E) \propto \exp \left[-E / E_{0}\right]
$$

dove $E_{o}$, dipendente dalla distanza geocentrica $r$, vale rispettivamente 400,120 e $6+\mathrm{keV}$ per $r=2,8,5$ e 6,1 raggi terrestri (fig. 5.20). Gli
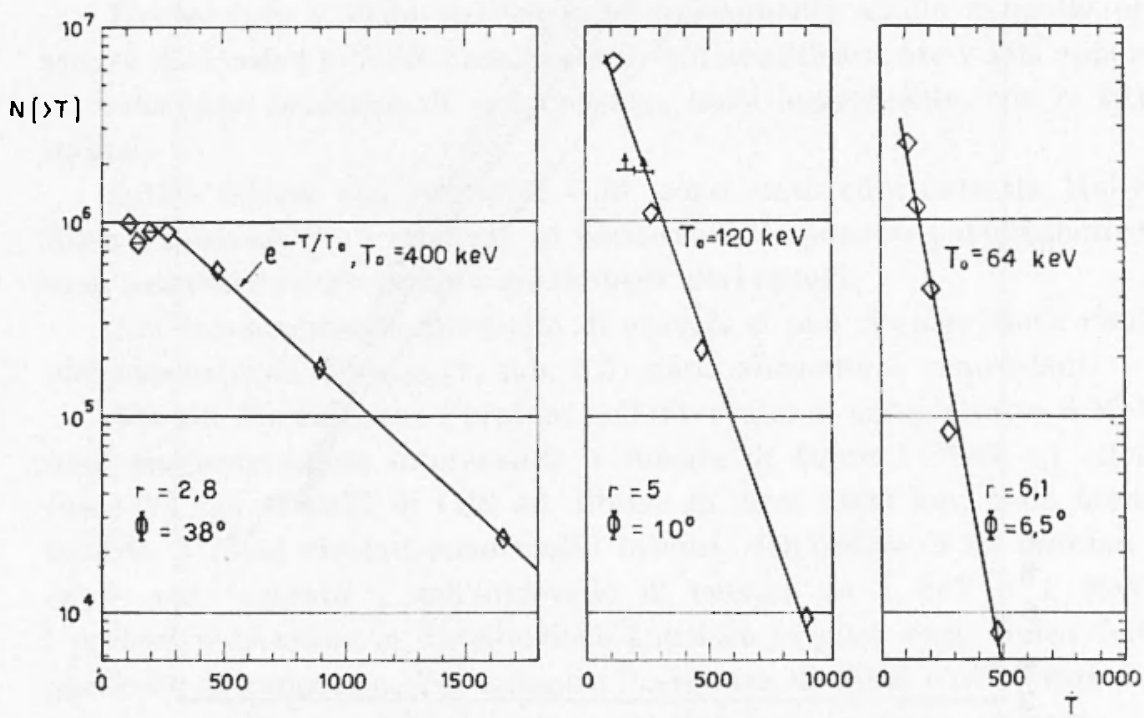

Pig. 5.20 - Spettro integrale di protoni (in protoni $\cdot \mathrm{cm}^{-2} \cdot \mathrm{sec}^{-1} \cdot \mathrm{sterad}^{-1}$ ) a varie distanze $r$ (in ragrgi terrestri) e alle latitudini geomagnetiche $\Phi$ indicate (secondo Davis e Williamson).

Autori hanno anche determinato la distribuzione equatoriale in pitch angle a approssimabile, nell'intervallo tra 0 e $72^{\circ}$, dalla funzione $\operatorname{sen}^{3} \alpha$.

Circa gli elettroni si hamo (fig. 5.19 e 5.21 ) le misure eftettuate al bordo inferiore della cintura esterna da Walt e coll. $\left({ }^{86}\right)$ e da Cladis e coll. ( $\left.{ }^{87}\right)$ mediante spettrometro magnetico inviato su razzo fino alla quota massima di $1045 \mathrm{~km}$, nell'intervallo di latitudini geomagnetiche 
$40^{\circ} \div 50^{\circ} \mathrm{N}$; nella salita fino a cirea $1000 \mathrm{~km}$ la traiettoria del razzo fu pressoché coincidente con la linea di forza magneticar attrarersante il piano equatoriale a distanza geocentrica di 2,4 raggi terrestri. In fig. 5.21

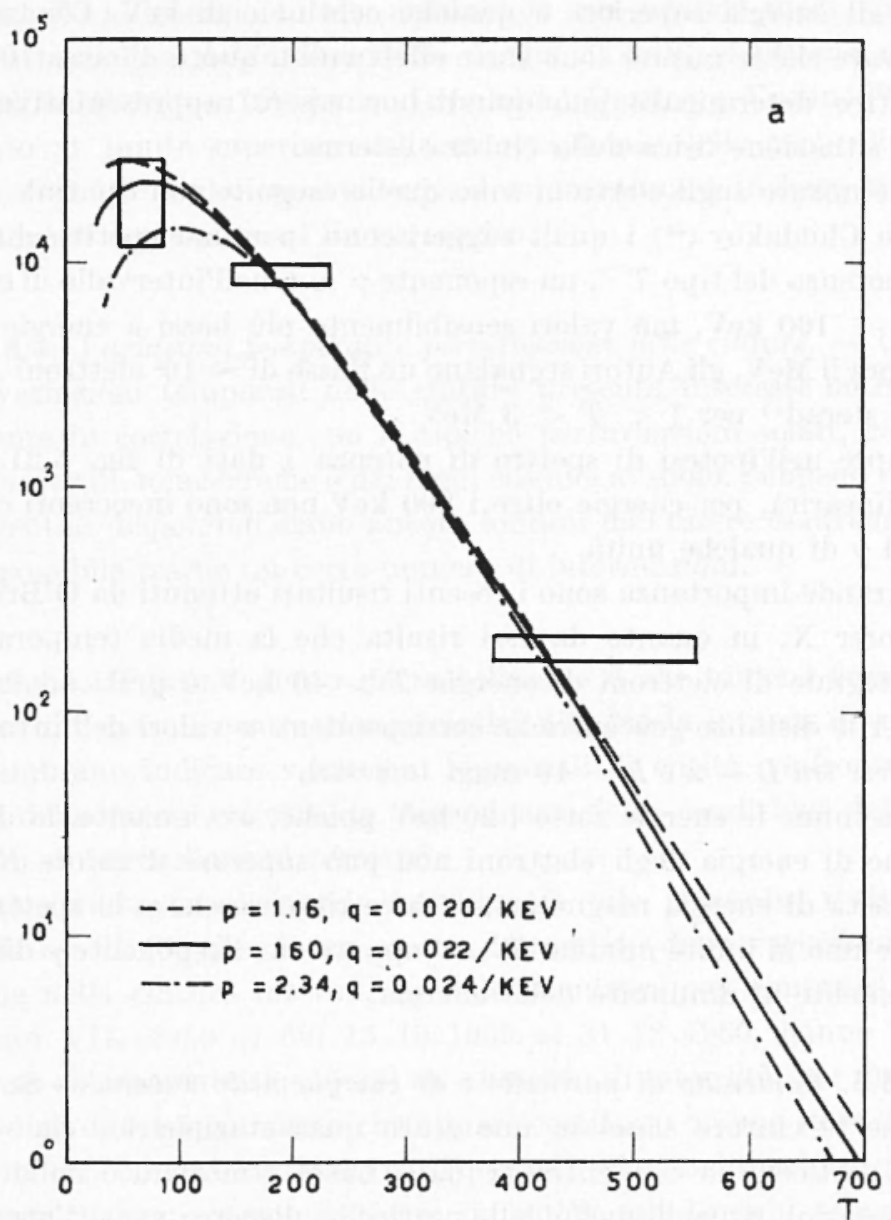

Fig. 5.21 ... Flusso differenziale omnidirezionale di elettroni, in elettro$n \mathrm{i} \cdot \mathrm{cm}^{-2} \cdot \mathrm{sec}^{-1} \cdot \mathrm{keV}^{-1}$; tra le varie coppie di valori $p$ e $q$, quella $p=1,60, q=0,022 \mathrm{keV}^{-1}$ rappresenta la migliore approssimazione; $T$, energia cinetica in $\mathrm{keV}$.

riportiamo il flusso integrale di elettroni misurato il $i$ luglio 1959 allik quota di $920 \mathrm{~km}$ e alla latitudine geomagnetica di circa $4 i^{\circ} \mathrm{N}$, flusso che ì approssimato nel modo migliore dalla funzione

$$
F_{0}(T) \propto T^{p} \exp \left[-q T^{\prime}\right]
$$

$$
\operatorname{con} p=1,60_{-, 74}^{0,74} ; q=0,022_{-0019}^{+0,0025} \mathrm{keV}^{-1} \text { per energie } \quad T>50 \mathrm{keV} \text {. }
$$


Lintensità omnidirezionale semple alla quota di $920 \mathrm{~km}$ (sempre per $T>50 \mathrm{keV})$ e risultata di $(3.4 \pm 0, \pi) \cdot 10^{6}$ elettroni $\cdot\left(\mathrm{cm}^{-2} \cdot \mathrm{sec}^{-1}\right.$.

Dai risultati citati appare molto chiammente la pratica assenza di elettroni di enereia superiore a qualehe eentinato di keV. Co tuttavia da osservare che le misure sono state eflettuate a quota di circa $1000 \mathrm{~km}$ e lo spettro determinato puó quindi non essere rappresentativo della eflettiva situazione fisica nella cintura esterna.

Altre misure sugli elettroni sono quelle eseguite con Sputnik III da Vernov e Chudakov ( $\left.{ }^{69}\right)$ i quali suggeriscono, per uno spettro differenziale di potenza del tipo $T^{-\bullet}{ }^{\prime}$, un esponente $\gamma \simeq 6$ nell'intervallo di energie $20<T<100 \mathrm{keV}$, ma valori sensibimente più bassi a energie superiori: sopra il MeV, gli Autori segnalano un flusso di $\sim 10^{\text {s }}$ elettroni $\cdot \mathrm{cm}^{-2}$ - sec ${ }^{-1} \cdot$ sterart $^{-1}$ per $1<T<3$ ML T.

Sempre nell’ipotesi di spettro di potenza i dati di fig. 5.21, nella zona di linearità, per energie oltre i 200 keV non sono incoerenti con un valore di $\gamma$ di qualche mitì.

Di grande importanza sono i recenti risultati ottenuti da O'Brien ${ }^{88}$ ) su Explorer X, in quanto da essi risulta che la media temporale del flusso integrale di elettroni di energia $T>40 \mathrm{keV}$ e praticamente costante per le distanze geocentriche corrispondenti a valori dell'invariante $L$ compresi tra $L=2$ e $L=10$ raggi terrestri.

Circa infine le energie sotto i $20 \mathrm{k}(\mathrm{T}$ poiché, ovvianente, la densita di volume di energia degli elettroni non puo superare il valore $B-/(8 \pi)$ della densita di energia magnetica, si deve ritenere che o lo spettro non si estende fino al limite minimo $T=0$ oppure che l'esponente $\gamma$ diminuisca fortemente al diminuire della energia.

5.3.3.3. Contenuto di particelle e di energia nelle cinture. - Se si ammette che le cinture siano in mo stato quasi-stazionario, che cioe il flusso di particelle in esse entranti (dalla bassa atmosfera o dallo spazio interplanetario) uguagli quello delle particelle disperse verso l'atmosfera terrestre alle medie e alte latitudini geomagnetiche, si può dare una valutazione del numero totale e dell'energia totale delle particelle intrappolate.

In base ai dati di tabella 5.2 si può inferire ma densità di particelle dell'ordine di una per $\mathrm{cm}^{3}$ con grandissima maggioranza di particelle di bassa energia: per confronto le densità di protoni di energia di qualche decina di MeT sono dell'ordine di solo $10^{-5} \div 10^{-6} \mathrm{~cm}^{-3}$. Il numero totale di particelle presenti nelle cinture si può perciò stimare dellordine di $10^{29}$ corrispondenti a una massa totale estremamente bassa. Se si 
assume, nel romplesso, una vita media delle particelle dellordine di $10^{6} \div 10^{7}$ secondi, si ha, nello stato quasi-stazionario, un flusso medio totale di particelle disperse dell'ordine di $10^{22} \div 10^{23}$ sece $^{-1}$.

Quanto all'energia totale, assumendo una energia media per particella intrappolata dellordine delle decine di keV, si ottiene un valore dell'ordine di $10^{14} \div 10^{15}$ joule. In realtà. Dessler e Vestine $\left({ }^{89}\right)$ hanno valutato il limite superiore della energia totale delle particelle intrappolate a $6 \cdot 10^{15}$ jonle; come si vede, il valore stimato in base ai risultati sperimentali è prossimo al limite teorico.

5.3.4. Fariazioni temporali e pertubazioni nelle cinture. - Lo studio delle variazioni temporali nelle cinture presenta interesse noterole spescialmente in correlazione con le tipiche perturbazioni solari, geomagnetiche, anrorali, ionosferiche e dei laggi cosmici al suolo. Sebbene i risultati sperimentali disponibili siano ancora lontani dall'essere esaurienti, è tu1tavia possibile trarne un rerto numero di informazioni.

5.3.4.1. Il primo punto da sottolineare è che almeno fino a questo momento, i dati di osservazione relativi alla fascia interna di van Allen non sembrano indicare variazioni temporali di entita confrontabile con quelle della fascia esterna, in dipendenza delle condizioni dellattività solare o di perturbazioni terrestri.

Sono tuttavia di grande interesses, a tale rignardo, i risultati di Pizzella e coll. $\left({ }^{90}\right)$ che, in un esame dettagliato delle variazioni a lungo termine nella cintura interma, secondo le misure con contatori G.M. su Explorer VII $\left(1959 \iota_{1}\right)$ dal 13.10 .1959 al 31.12 .1960 , hamno messo in evidenza chiamanente (fig. 5.22) un aumento di intensità per un fattore dell'ordine di $2 \div 3$, ammento che appare costituito da una serie di bruschi aumenti concomitanti con arrivi di protoni solari nelle calotte polari e/o con perturbazioni geomagnetiche; in realtà tali bruschi aumenti sono ben netti a distanze $L>2$, intermedie tra fascia interna e fascia esterna (fig. 5.23), ma sono individuabili anche nelle misure ottenute per valori più bassi di $L$, cios̀ ben dentro la cintura interna. Nol complesso la forma della fascia non risulta modificata nei 15 mesi di osservazione, e questo anche nei periodi perturbati.

Queste osservazioni suggeriscono la presenza di una sorgente addizionale di particelle oltre quella del decadimento dei neutroni di albedo che nel periodo considerato non può essere variato più di quel $20_{0}^{\circ}$ di cui al massimo è variata $\left({ }^{91}\right)$ l'intensità dơi raggri cosmici primari. 
5.3.4.2. Assai più sensibile alle perturbazioni risulta invece la cintura esterna; un esempio imponente è già ben visibile sui primi dati raccolti (fig. 5.14a); mentre la intensità misurata nella cintura interna non presenta differenze troppo rilevanti tra le due date del 6 dicembre 1958 a del 3 marzo 1959, risultano invece fortemente diverse la posizione e l'intensità nella cintura esterna; inoltre, a distanza tra 10 e 15 raggi terrestri,

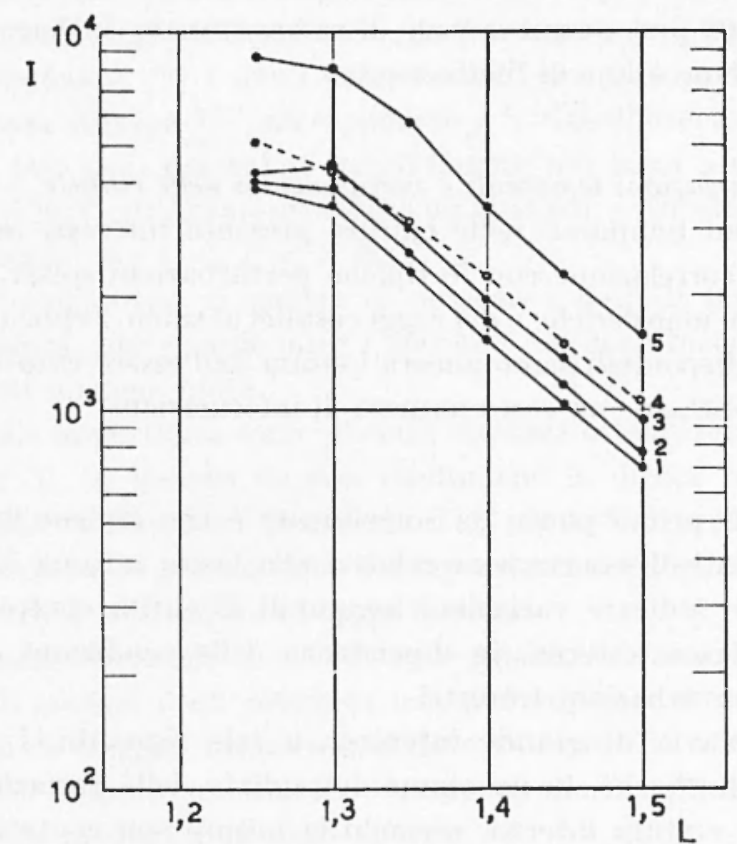

Fig. 5.22 - I, intensità medie (in conteggi/sec.) a varie distanze $L$ (in raggi terrestri); le curve indicate con i numeri $1,2, \ldots, 5$ si riferiscono rispettivamente ai periodi 13 ottobre 1959-31 maryo 1960, aprile 1960, maggio 1960, gingno-15 novembre 1960 e 16 novembre-31 dicembre 1960 (secondo Pizzella, McIlwain e van Allen).

Pioneer IV ha rilevato una fitta serie di massini e minimi. Con riferimento alle condizioni esistenti nei giolni di lancio e in quelli immediatamente precedenti, il volo ja Pioneer III avvenne in un periodo di calma geomagnetica, mentre il volo di Pioneer IV fu preceduto da una tempesta magnetica (con iniziojbrusco alle 0215 T.M.G. del 25 febbraio 1959) e da forte attività aurorale nelle notti del 25, 26, 27, 28 febbraio e 1 marzo.

Una situazione della cintura esterna analoga a quella riscontrata da Pioneer III fu anche segnalata da Vernov e coll. $\left({ }^{02}\right)$ in base alle osservazioni di Lunik I, pure in un periodo di calma geomagnetica. 

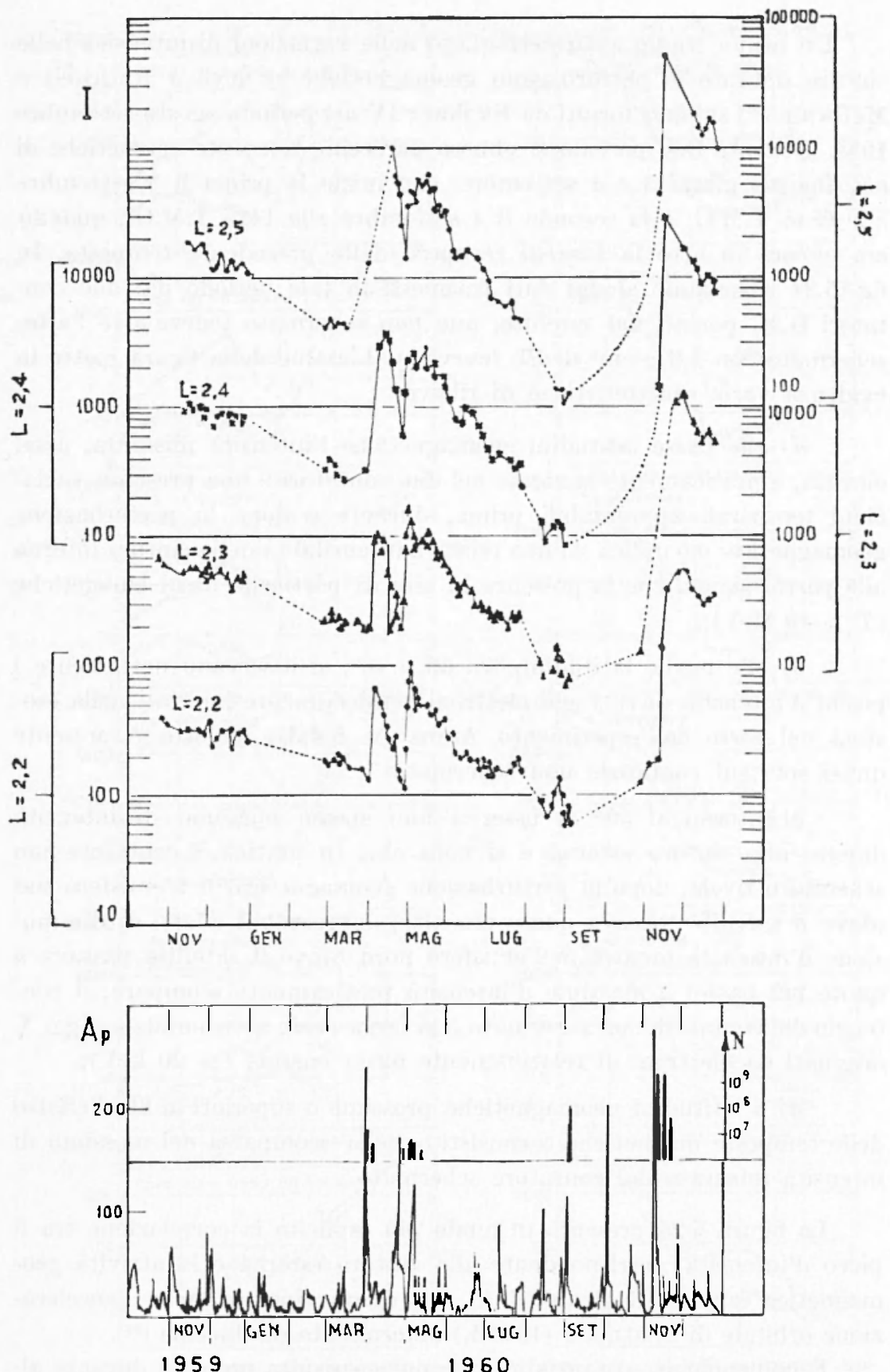

Figr. 5.23 - $I$, intensita medie misurate (in contegrisec) a varie distanze $l$. (in ragri terrestri); $\Lambda_{p}$, indice di attivita magnetica: $N$. numero totale di protoni solari (in $\mathrm{em}^{-2}$ ) affluiti in varie occasioni (secondo P'izzella, Micllwain e van Allen). 
Vn primo studio assai dettagliato delle variazioni di intensità nelle rinture durante le perturbazioni geomagnetiche si deve a Rothwell a If. Ilwain $\left({ }^{93}\right)$ sui dati forniti da Explorer IV nel periodo agosto-settembre 1958. Durante tale periodo si ebbero parecehie tempeste magnetiche di cui due nei giomi 3 e 4 settembre, con inizio la prima il 3 settembre alle 0843 T.MI.G. e la secomda il 4 settembere alle 1415 T.M.G., quando era ancora in atto la fase di recupero della precedente tempesta. In fig. 5.24 riportiamo alcuni dati trasmessi in tale periodo dai dne contatori G.Mr. portati dal satellite, uno non schermato (curve a) e l'altro schermato con $1,6 \mathrm{~g} / \mathrm{em}^{2}$ di Pb (curve b). L'esame della figura mette in evidenza varie caratteristiche di rilievo:

a) alle basse latitudini geomagnetiche l’intensità misumata, assai elevata, ì praticamente la stessa nei due contatori e non presenta rariazioni temporali apprezzabili prima, durante e dopo la perturbazione geomagnetica; ciò indica sia ma relativa insensibilità della cintmra interna alle perturbazioni che la presenza in essa di particelle assai energetiche $(T>40 \mathrm{M}(\mathrm{V})$;

b) alle medie latitudini, tla 30 a $40^{\circ}$, si osservano nettamente $\mathrm{i}$ picchi d'intensità dovuti agli elettroni artificialmente iniettati nella esosfera nel corso dell'esperimento dreus (v. 5.4.3); l'effetto è presente quasi solo sul contatore non schermato;

c) intorno a $50^{\circ}$ si osserva uno spesso massimo di intensita dovuto alla rintura estema e si nota che, in pratica, il contatore nom schermato rivela, dopo la perturbazione geomagnetica, nell'emisfero sud (dove il satellite toceara quote più alte) apprezzabili effetti di diminuzione d'intensità mentere nellemisfero nord (dove il satellite passava a quote più basse) il massimo d’intensità praticamente seompare; il conteggio del contatore non schermato ì in larga parte attribuibile a raggi $X$ orig̣nati da elettroni di relativamente bassa energia (> 20 keV);

d) a latitudini geomagnetiche prossime o superiori ai $60^{\circ}$ lefefetto delle tempeste magnetiche è consistito nella scomparsa del massimo di intensità misurato dal contatore schemato.

La figura 5.25 presenta in modo più esplicito la correlazione tra il piceo d'intensità corrispondente alla cintura esterna e la attività geomagnetica espressa dagli indici $K_{p}$. I a figura riporta anche l'accelerazione orbitale di Sputnik I (1958 $\left.\delta_{1}\right)$ determinata da Jacehia $\left(^{9.4}\right)$.

Fenomenologia sostanzialmente analoga risulta presente durante altre tempeste. 

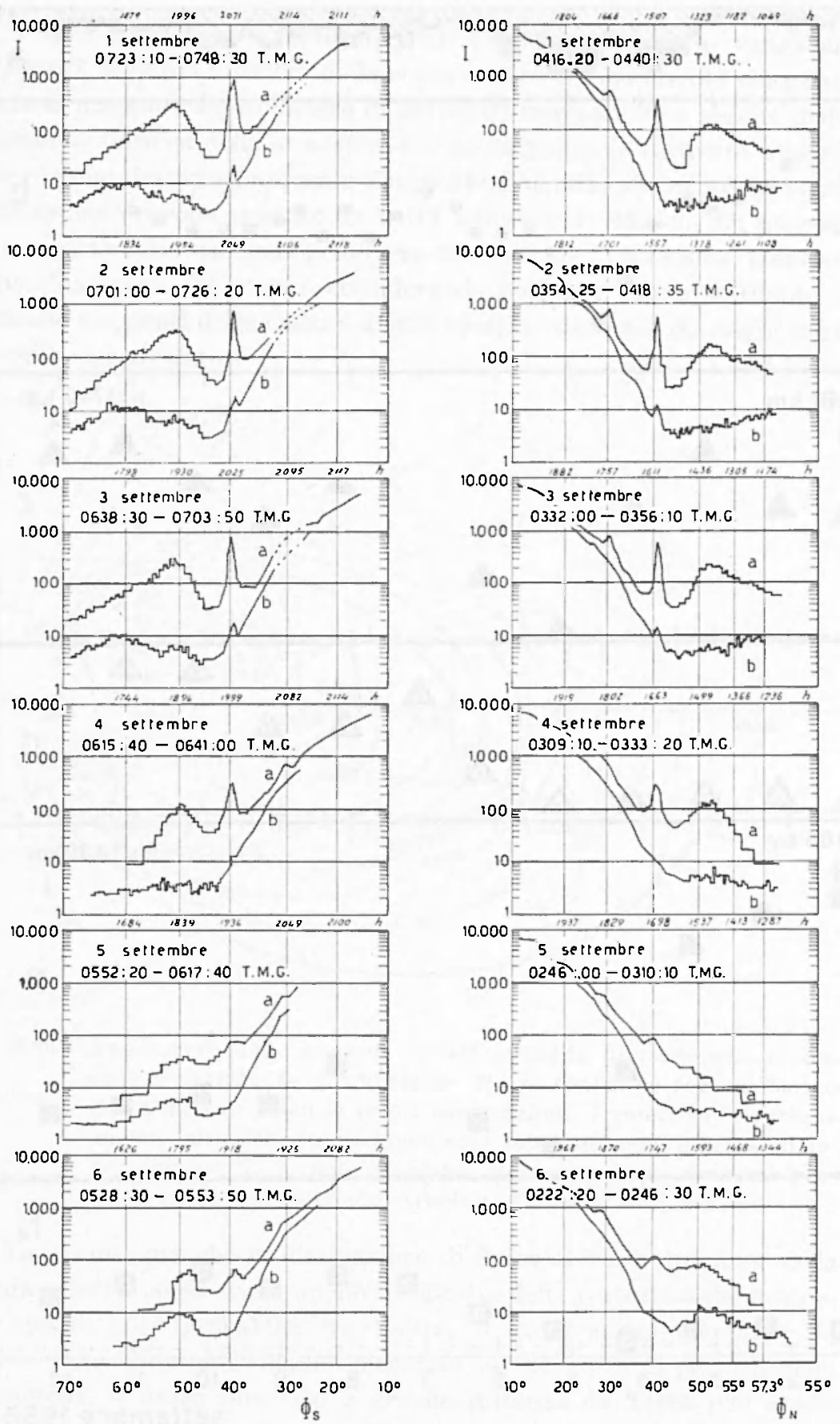

Tigr. 5.24 - I, intensità di conteggio in impulsi/sec: $\Phi_{\mathrm{N}}$, $\Phi_{\mathrm{s}}$. latitudine geo. magnetica nord e sul: h, altezza del satellite sul suolo. Per ciascma coppia di grafici è indicato l'intervallo temporale !di misurazione (secondo Rothwell e Mr.Ilwain). 


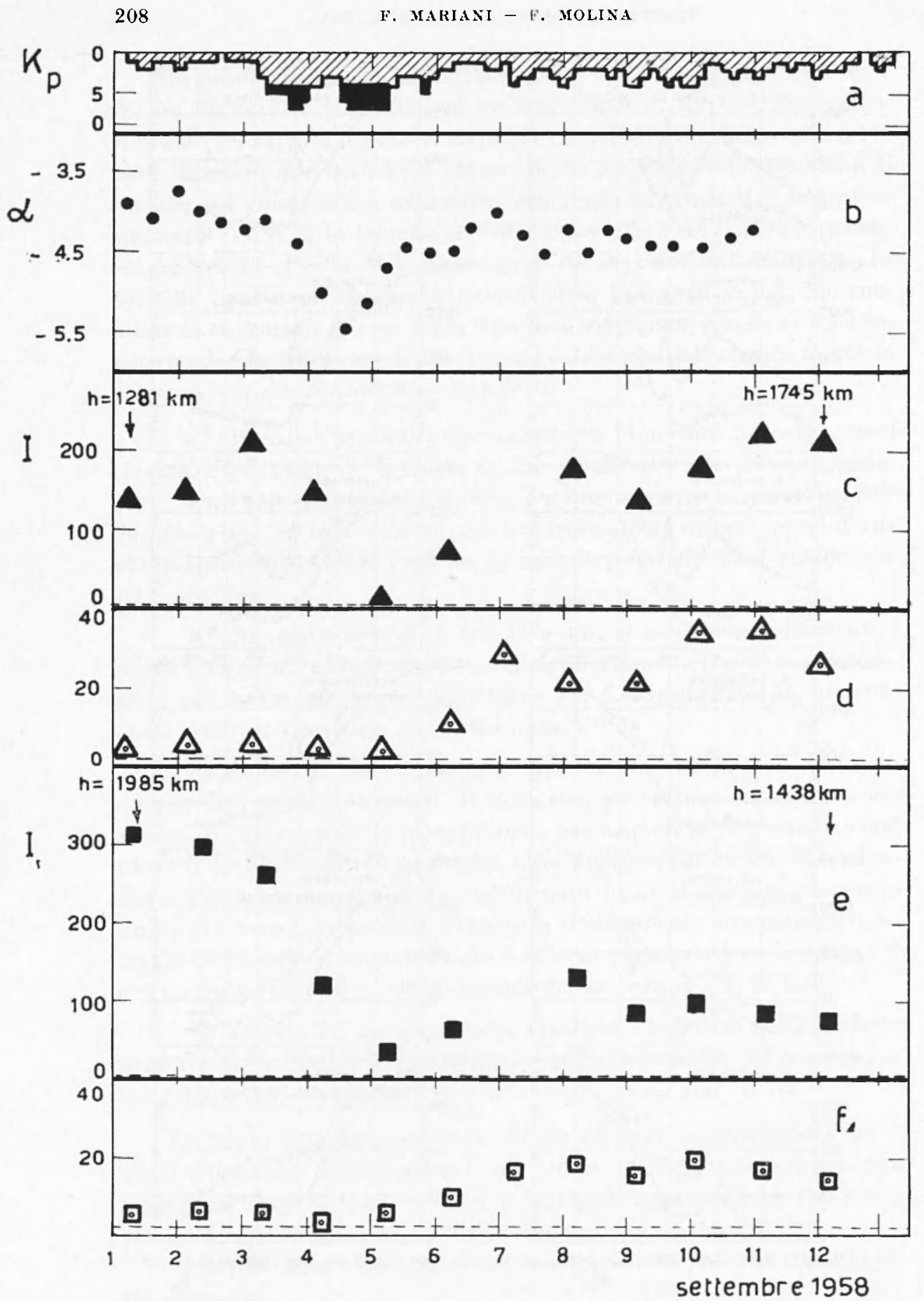

Fig. $5.25-K_{p}$, indice magnetico planetario (fig. $a$ ); $a$, accelerazione orbitale di Sputnik I in unità $10^{-5} \mathrm{~m} / \mathrm{sec}^{-2}$ (fig. b); $I$, intensità massima in impulsi/sec. misurata dal contatore (i.M. non sehermato e risp. da quello sehermato sul Nord-America (figg. $c, d$ ) e sull'Australia (figg.e. $f$ ); sono indicate le altezze $h$ di Fxplorer IV allinizio e alla fine del periodo di misurazione: sulle ascisse la data di Greenwioh (secondo Rothwell e Irllwain). 
I dati sperimentali sopra riportati suggeriscono whe le variazioni d'intensità al bordo inferiore della cintura esterna sono dovute essenzialmente al maggiore assorbimento di particelle intrappolate a seguito della dilatazione termica della atmosfera che accompagna le tempeste magnetiche e le manifestazioni auromali: l'amento di densità alle quote dei punti di riflessione provoca appunto un netto aumento di efficacia dei processi di scomparsa delle particelle, cioè una diminuzione di intensità. L'elletto di riscaldamento che stiamo considerando potrebbe essere attribuito a particelle sfuggenti dalle cinture aventi eneroia cosi bassa da essere sotto la soglia dei rivelatori.

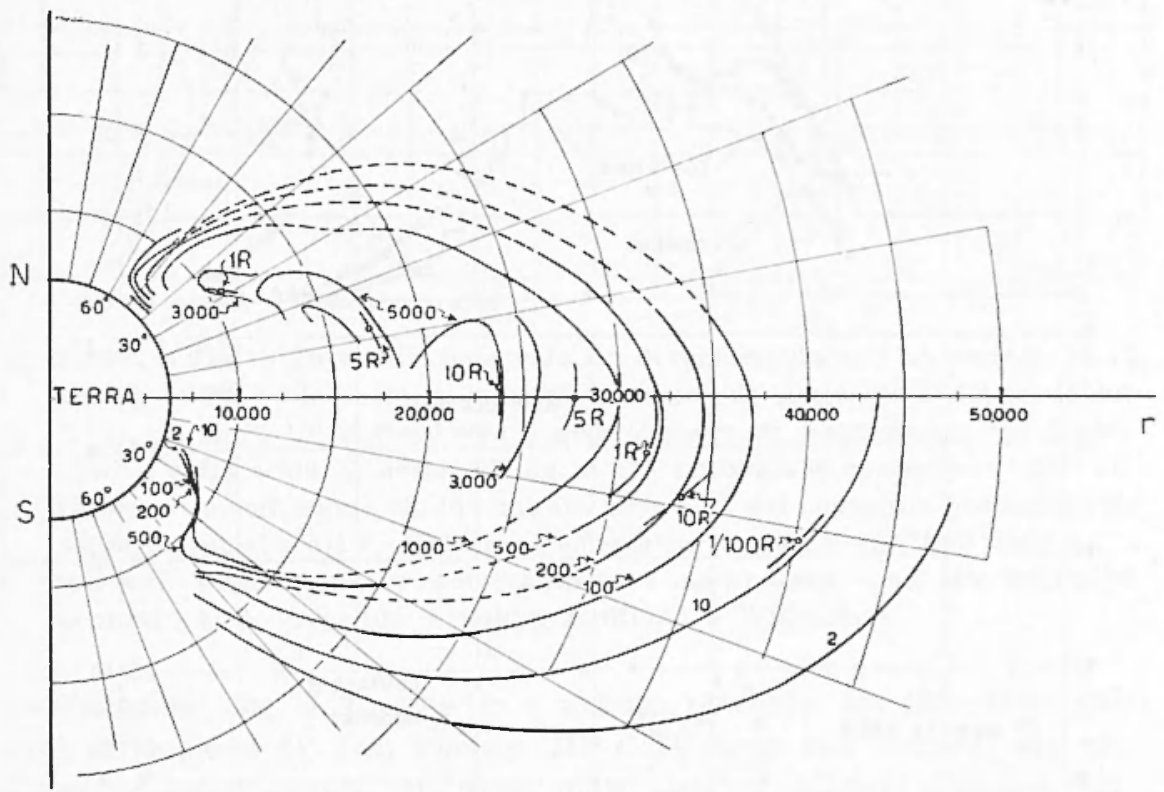

Fig. 5.26 - Andamento delle linee di uguale intensità di conteggio, in impulsi/sec, ottenute con lo stesso tipo di contatore con cui furono fatte da van Allen le prime osservazioni. I punti -o- riportano anche intensita (in roentgen/ora) misurate con camera di ionizzazione: N. S, poli greografici nord e sud: $r$, distanza geocentrica in $\mathrm{km}$ (secondo Arnoldy, Ioffiman e Winckler).

Ia eventualità che la dininuzione di flusso al bordo inferiore della cintura esterna possa essere un effetto diretto della perturbazione magnetica appare poco probabile; tra l'altro, le osservazioni compinte da Pioneer III e Pioneer IV hanno mostrato che nel corso di perturbazioni magnetiche, il flusso misurato a grande distanza da Terra puó anche aumentare. 

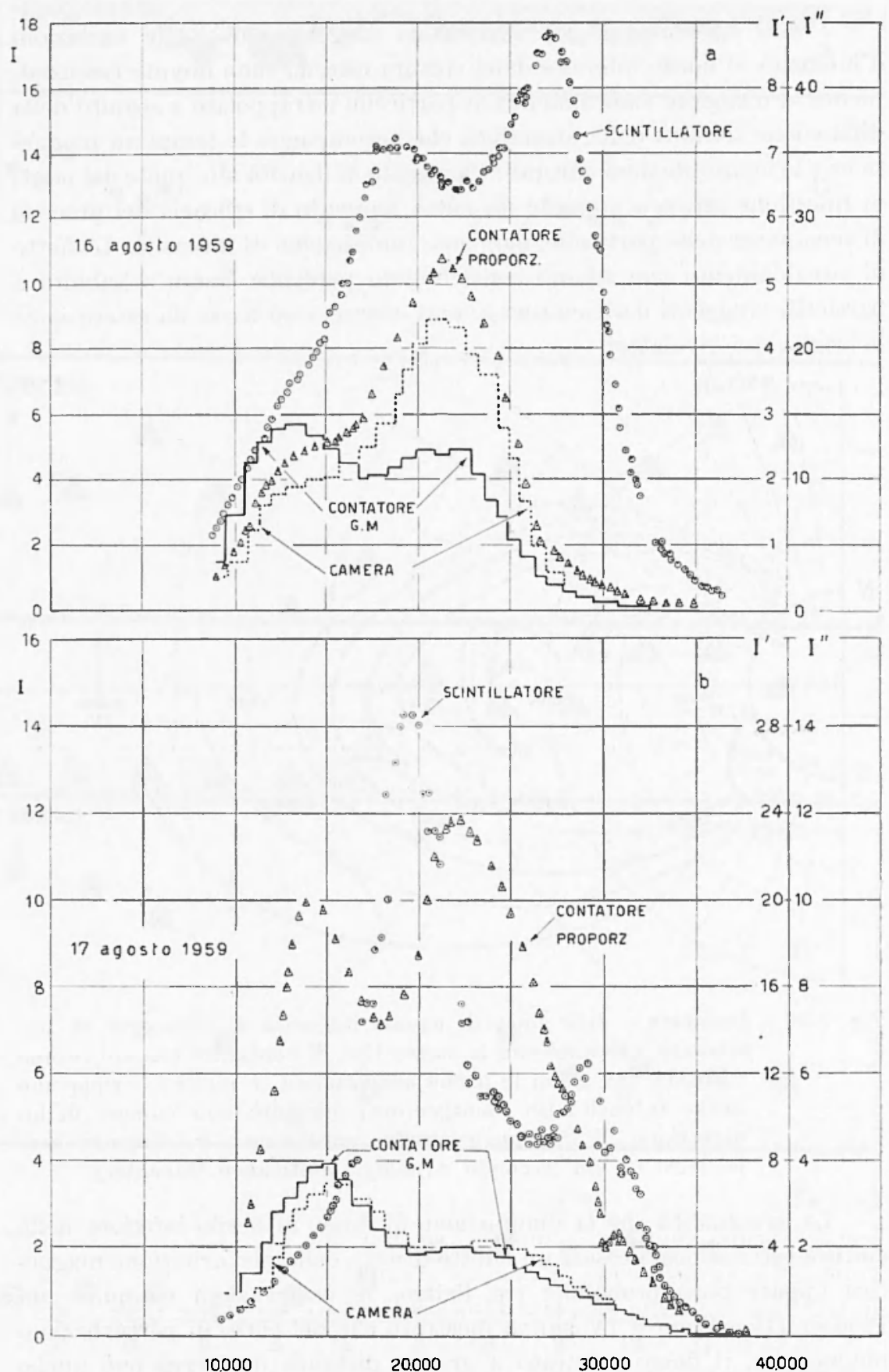

Fig. $5.27 a, b$ 
Ulteriori osservazioni sulla radiazione nelle cinture in periodi sia magneticamente calmi che perturbati sono dovute a Arnoldy, Hofiman a Winckler $\left({ }^{95}\right)$ su Explorer VI nel periodo tra il 7 agrosto e il 6 ottobre 1959. Dalle misure ottenute in giomi calmi appaiono nettamente notevoli
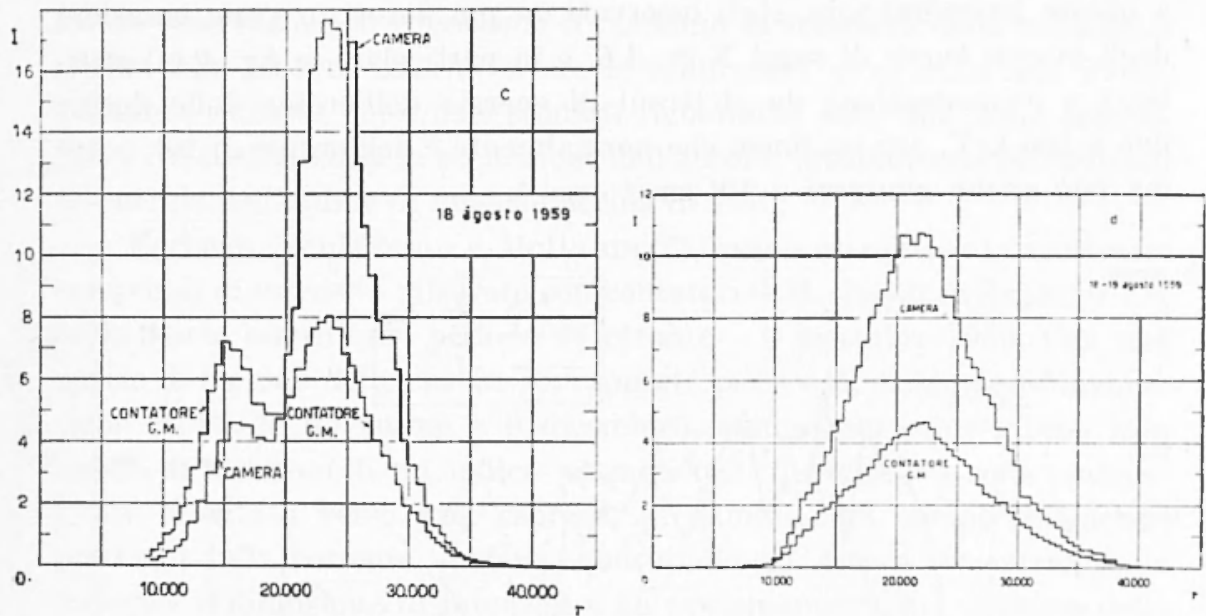

Hig. $5.27 c, 5.27 d$ - Intensiti registrata da diversi rivela tori nel periodo 16-19 agosto 1959. Sulla scala di sinistra intensita di conteggio $I$ del contatore G. M. (in unita $10^{3}$ impulsi/sec) e della camera di ionizzazione (in impulsi/sec); sulla scala di destra di fig. $a$ e $b$ intensita di contegrorio $I^{\prime}$ del contatore a scintillazione (in $10^{5}$ impulsi/sec) e $I^{\prime \prime}$ del contatore proporzionale (in $10^{2} \mathrm{impulsi/sec);} r$, distanza geocentrica in $\mathrm{km}$; i risultati delle fig. $c$ e $d$ sono stati ottenuti rispettivamente a molto basse e ad alte latitudini geomagnetiche (secondo Arnoldy, Hoffman e Winckler)).

modificazioni (fig. 5.26) rispetto a quanto ottenuto da van Allen sui dati di Explorer IV a di Pioneer III e IV sono ben evidenti sia un sensibile arvicinamento alla Terra della zona di massima intensità, sia un notevole cambiamento di forma e in particolare un "appiattimento" sul piano equatoriale geomagnetico, per cui i "corni" della cintura esterna ranno a insistere a latitudini più basse. Quanto alle misure in condizioni perturbate $\grave{e}$ di interesse lo studio dei dati raccolti a cavallo della tempesta magnetica a inizio brusco cominciata alle 0404 T.M.G. del 16 agrosto. Al forte effetto di svuotamento (fig. $5.27 a, b$ ) presente alla fine del primo giomo di tempesta, rivelato dal contatore G.M. e dalla (anera, fa seguito verso la fine del secondo giorno un netto aumento, sia alle basse che alle alte latitudini geomagnetiche (fig. 5.27 c,d).

Dal confronto tra le diminuzioni d'intensità osservate dal contatore e dalla camera, gli autori attribuiscono lo sruotamento a perdita di 
elettroni di energia dellordine dej $40-50 \mathrm{keV}$, in corrispondenza alle atitudini geomagnetiche tra 52o a 620 ; l'effetto è massimo intorno ai $57^{\circ}-58^{\circ}$. Si noti che queste latitudini sono sensibilmente inferiori a quelle a cui è normalmente presente l'aurora polare a che, d'alt la parte, proprio a queste latitudini sono stati osservati da più autori in varie occasioni degrli intensi bursts di raggi $\mathrm{X}$ (v. 4.6. e in particolare la fig. 4.44) attribuiti a bremsstrahlung da elettroni di energia dell'ordine delle decine fino a $100 \mathrm{keV}$, con un flusso che normalmente a dell'ordine di $10^{5} \div 10^{6}$ ma può anche giungere a $10^{8} \mathrm{CHL}^{-2} \cdot \mathrm{sec}^{-1}$.

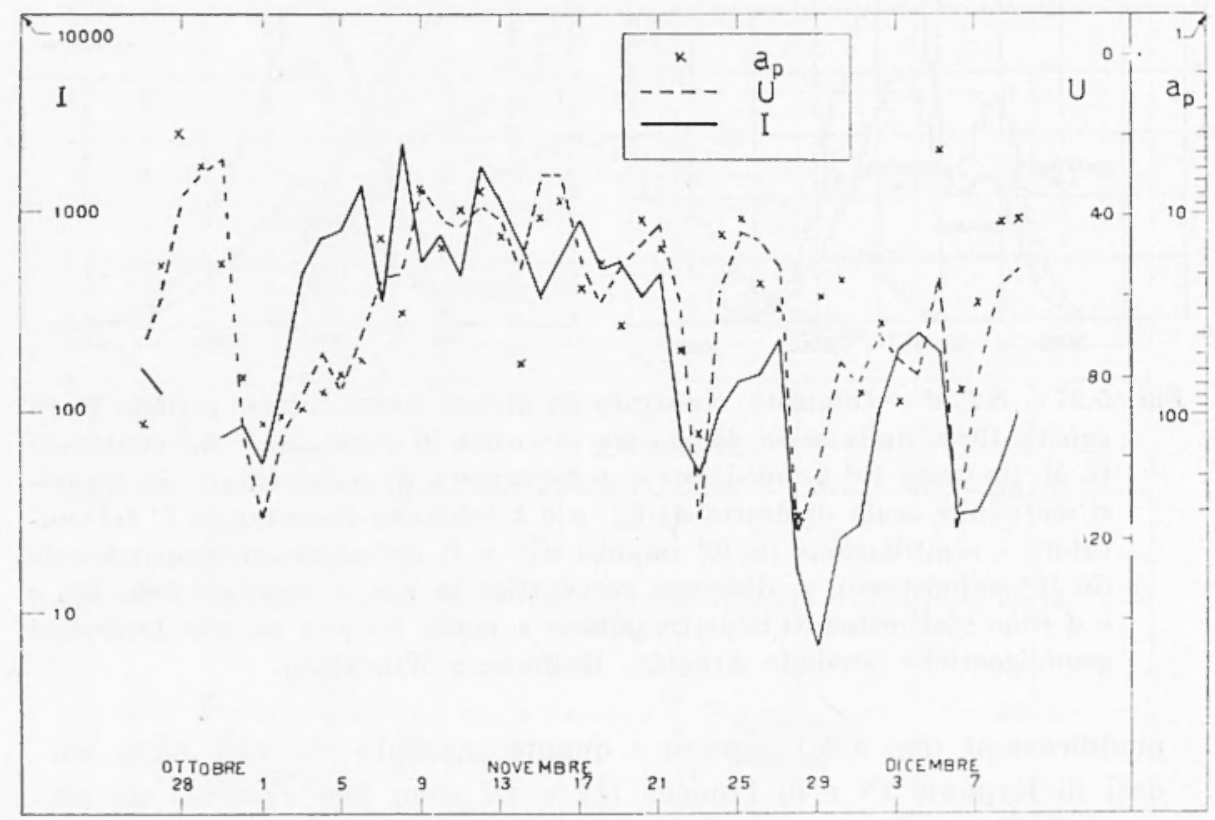

Fig. 5.28 - I. intensita di conteggio; $t$ e $a_{p}$, indici magnetici. in gamma (secondo Forbush, Venkatesan e Mcllwain).

Dal punto di vista quantitativo, dai dati di osservazione si deduce una lapidita di svuotamento della cintura esterna nel corso della tempesta magnetica che è prossima a quella che si valuta attribuendo agli elettroni dispersi l'origine dei bursts di raggi $\mathrm{X}$. Ta detto ancom che la notte tra il 16 e il 17 agosto fu visibile sul Minnesota (lat. geomann. 57o $\mathrm{N}$ ) un'aurora a struttura raggiata, del tipo di quella considerata nella citata fig. 4.44 .

Più o meno nella stessa epoca, Anderson $\left({ }^{96}\right)$, sulla base di osservazioni di raggi $\mathrm{X}$ su palloni fatte in zona aurorale, giunge alla conclusione 
che sebbene una intensa ladiazione $\mathrm{X}$ fosse osservabile nel corso di aurore visuali arrenute a lat it udini inferiori a quelle aurorali, alle nomali latitudini aurorali tuttavia la radiazione $\mathrm{X}$ risultò molto debole: ciò corrisponde con tutta probabilità al fatto sopra rilevato che nellepoca cui le osservazioni si riferiscono il massimo di intensità della laddiazione molle della cintura esterna si era sensibilmente avricinato alla Terra. Notiamo tuttavia che i dati studiati riguardano solo ma delle componenti cui si attribuisce la eccitazione dell'a urora, precisamente gli elettroni di eneregia dell'ordine di qualche decina di keV.

Forbush, Venkatesan e Mr(Ilwain $\left({ }^{97}\right)$ hanno esaminato le variazioni temporali di intensità misurate con contatori G.M. singoli su Explorer VII nella fascia esterna nel periorlo 26 ottobre - 9 dicembre 1959 . Con ma specie di metodo delle epoche sovrapposte per 4 casi di tempeste magnetiche $(1,23,28$ novembre e 6 dicembre), assumendo come giorno zero quello di massino di un indice magnetico $U$ (definito $\left({ }^{98}\right)$ come componente orientata rerso sud, espressa in gamma, del campo magnetico prodotto dalla corrente anulare equatoriale) gli Autori riscontrano una notevole diminuzione di intensità e un avicinamento del massimo della fascia esterna nei giomi 0 e +1 con un completo ripristino al quinto giorno. Sembra anche presente (fig. 5.28) una debole correlazione negativa tra indici magnetici $C$ e $a_{p}$ da una parte e conteggio del contatore a distanza $L=4,1$ laggi ter'estri, dall'altra.

La diminuzione di intensità per grandi ap alle distanze più grandi corrispondenti alla parte esterna della cintura ì consistente con l'idea che le particelle vadano a cadere alle alte latitudini geomagnetiche.

E interessante confrontare le misure ottenute con contatore e camera di ionizzazione con quelle effettuate da Rosen e coll. $\left({ }^{99},{ }^{100}\right)$ mediante contatore a scintillatore plastico sensibile a elettroni e protoni di energia rispettiramente superiore a $200 \mathrm{keV}$ a $2 \mathrm{MeV}$, ma assai poco sensibile alla bremsstrahlung. In coincidenza con la tempesta del 16 agosto lo scintillatore segnalò (fig. 5.27 $a, b$ ) un notevole ammento di conteggio, a differenza degli altri rivelatori che indicarono forte diminuzione. Per molti gionini, fino a un suceessivo aumento di attività magnetica, l'intensità andó declinando lentamente mantenendosi notevolmente più alta di quella "pre-tempesta ». Nei giorni di tempesta lo scintillatore segnalava anche, a distanze geocentriche oltre $40.000 \mathrm{~km}$, cioè al bordo esterno della fascia, rapide variazioni di intensità di ampiezza fino a $2 \div 3$ ordini di grandezza in intervalli di tempo da qualche secondo a qualche minu10 (fig. อ̆.29). 

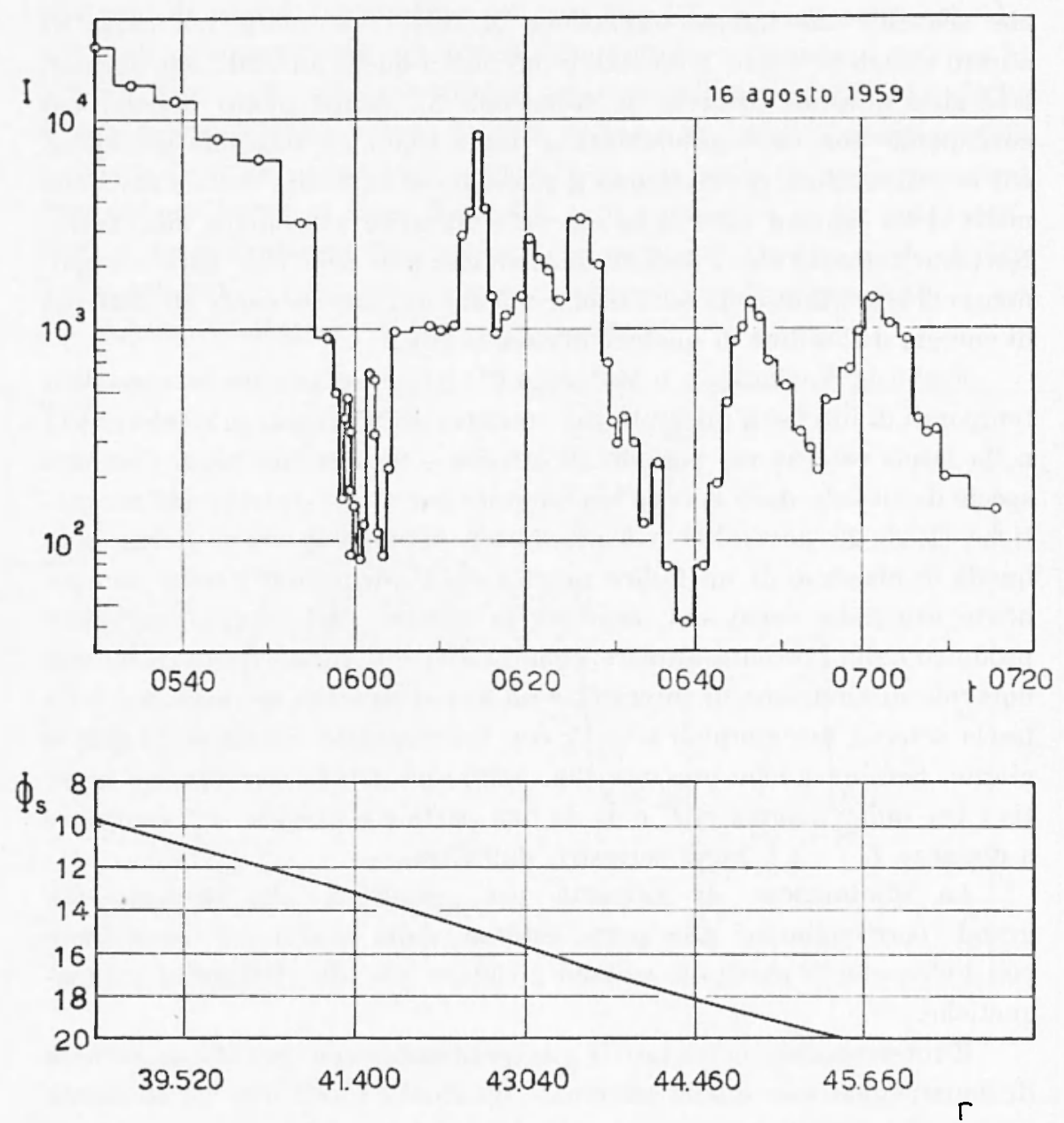

Fig. 5.29 - $I$, intensita di contegrgio in impulsi/sec; $\Phi_{s}$, latitudine geomagnetica sud di Explorer VI; $t$, tempo medio di Greenwich: $r$. distanza geocentrica in $\mathrm{km}$ (sconclo Rosen. Farles e Sonett).

Gli aumenti connessi a queste ultime futtuazioni sono attribuibili con tutta probabilità a un meccanismo di accelerazione, tipo betatrone, che porta l'energia di elettroni molli al di sopra della soglia dello scintillatore, senza però un reale aumento della eflettiva intensità elettronica totale, come del resto suggerisee il responso degli altri strumenti particolarmente sensibili alla bremsstrahlung i quali non rivelano nessuna confrontabile variazione di conteggio. Se questa interpretazione è vera si può prevedere una variazione di intensità in fase con la variazione del 
campo magnetico: in effetti questo è realmente avvenuto in parecchi (asi (101). Tuttavia durante la tempesta magnetica del 16 agosto la variazione di intensità della radiazione corpuscolare è in netta opposizione di fase (fig. 5.30) con quella del campo magnetico $\left({ }^{102}\right)$ misurato localmente dal satellite.
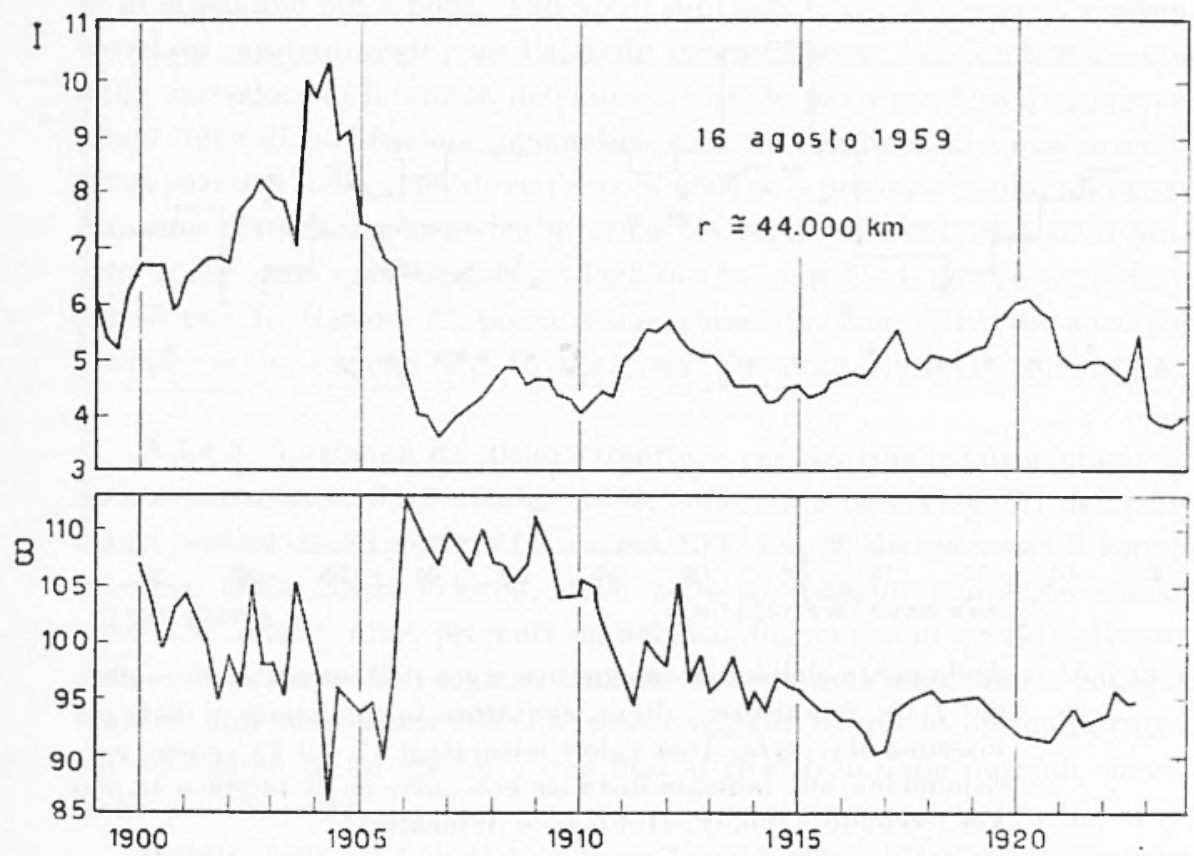

Fig. 5.30 - $I$, intensità di conteggio (in $10^{3}$ impulsi/sec); $B$, intensità del campo magnetion in gamma; $t$, tempo medio di Creenwich: $r$ distanza geocentrica (secondo Farler e Rosen).

I dati di Explorer VI sono anche interessanti in quanto permettono di determinare, in corrispondenza all'apogeo $(\sim 48.000 \mathrm{~km})$, le intensita di ladiazione al bordo esterno della cintura (fig. 5.31). Appare chiamamente la presenza di due periodi, l'uno in concomitanza alla perturbazione magnetica del 16 agosto e l'altro tra il 27 e il 28 agosto, in cui l'intensita fu ben superiore a quella "normale " dei giorni precedenti. Assai incerto è il significato fisico di tali aumenti: potrebbe trattarsi di radiazione corpuscolare iniettata direttamente dal Sole, come pure di radiazione di energia inizialmente così bassa da non poter essere rilevata dagli strumenti di misura ma poi accelerata al disopra della soglia degli strumenti stessi da qualehe mecamismo locale di accelerazione. Nel caso della tempesta 
del 16 agosto tale processo sembla coincidere con il ritorno del campo magnetico al suo valore normale, cioè con la scomparsa del campo $D_{s t}$.

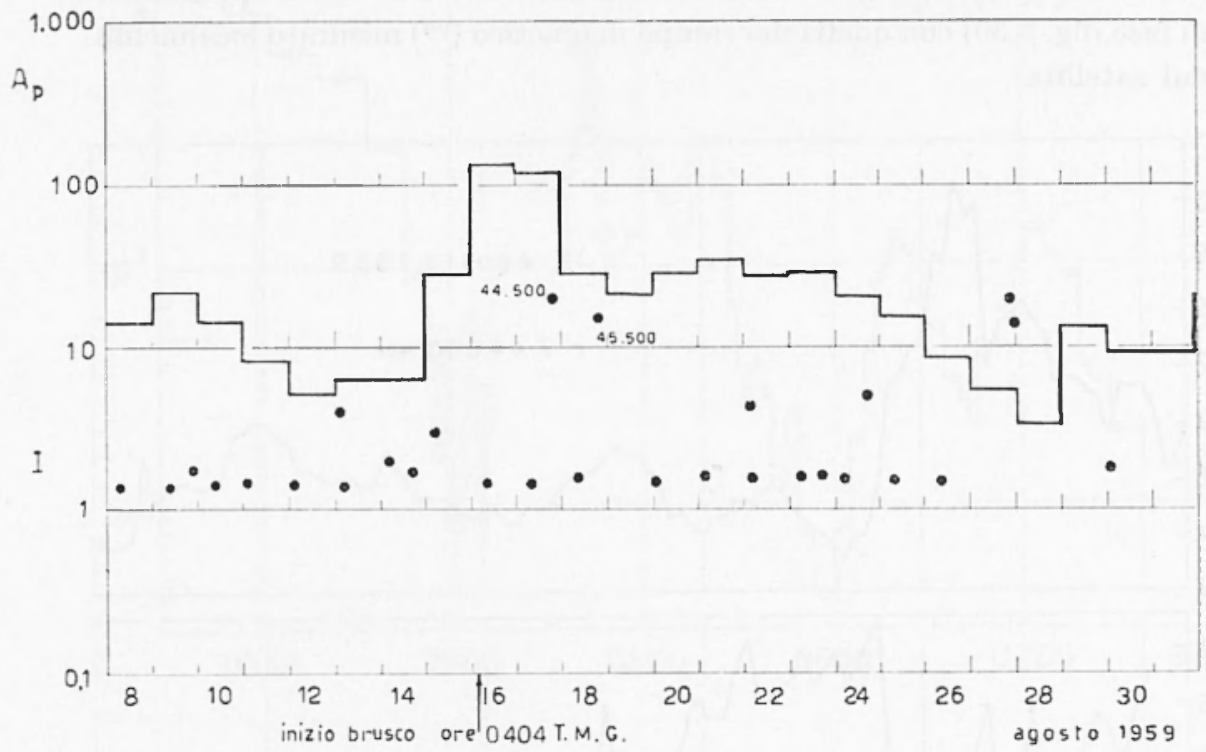

Fig. 5.31 - Andanento dellindice magnetico $A_{p}$ e dell intensita di conteggio I (in impulsi/sec) di nu contatore in condizioni di distanza massima da Terra. Due valori misurati il 17 e il 18 agosto corrispondono alle indicate distanze geocentriche di $\mathbf{4 . 5 0 0}$ e $\$ 5.500$ km (secondo Arnoldy, Hoffman e Winckler).

Sono ancora da segnalare alcune interessanti rilevazioni dorute a Fan, Meyer e Simpson $\left({ }^{72}{ }^{103}\right)$, relative ai due massimi di intensità elettroniar $E_{2}$ ed $E_{3}$ : mentre il massimo $E_{3}$ subisce dopo la tempesta magnetica del 16 agosto un abbassamento, sia pure ritardato di qualche giorno rispetto alla perturbazione, e si sposta di qualche migliaio di $\mathrm{km}$ verso la Terra (fir. 5.32), il massimo $E_{2}$ appare praticamente insensibile, entro un fattore 2 nella intensità, alla attività geomagnetica. Questo diverso comportamento per i due massimi suggerisce diversa origine e diverso mecemismo di seomparsa degli elettroni nelle regioni interessate: in ogni caso risulta provato che la vita media degli clettroni nella regione più esterna è abbastanza lunga, dell'ordine di parecehi giorni. Comunque la successiva tempesta magnetica del 3 settembre 1959 non abbe efietto apprezzabile sul massimo esterno; ciò mostra conte non tutte le tenpeste abbiano necessariamente gli stessi effetti. 
In un recente studio sulla fenomenologia delle due regioni $E_{2}$ ed $E_{3}$, Stolov (104) fa notare che vari autori hanno osservato fotometricamente (non visibihmente) la frequente occorrenza di un arco aurorale in luce di 6300 Å di assai debole intensità, a quote intorno ai $400 \mathrm{~km}$, in occasione di simultanee manifestazioni aurorali visibili manifestantesi circa $8^{\circ}$ di latitudine più a nord. Tali archi subrisibili, la cui presenza sembra correlata positivamente con l'attività geomagnetica, indipendentemente dalle variazioni di intensità dell'aurora visibile più a nord, si dispongono lungo linee di inclinazione magnetica costante; le linee di forza magnetiche passanti nella zona dove l'arco a 6300 \& è presente hamno allequatore una distanza geocentrica di circa 2,5 laggi terrestri, ciò sono proprio quelle sulle quali appare "adagiata" la zona $E_{2}$. L'autore suggerisce perciò che la regione $E_{3}$ possa essere correlata con l'attivita aurorale visibile e la regione $F_{2}$, invece, con l'attività aurorale subvisibile.

5.3.4.3. Vogliamo da ultimo riportare che, in condizioni manneticamente perturbate, il 18 ottobre 1959 , sono state osservate (105) dai contatori portati da Explorer VIT, a circa $1000 \mathrm{~km}$ di altezza rerso il lembo inferiore della fascia esterna, delle zone di maggrior conteggio spesse circa $0,5^{\circ}$ di latitudine, presenti sia nel lato diurno che in quello notturno della Terla, zone che progressivamente si sono mescolate fino a confondersi in una zona unica con intensità maggiore di quella nomale; tutto ciò nel tempo di un giorno o due fino al ripristino delle normali condizioni pre-tempesta.

Queste zone di maggior conteggio sono parse attribuibili secondo gli autori a particelle intrappolate con vita media di poche ore.

5.3.4.4. Nei paragrafi precedenti abbiamo considerato qualche aspetto della associazione fra cinture di van Allen e fenomeni aurorali. Vogliamo ora considerare la questione con qualche maggiore dettaglio. Di fatto ci sono argomenti per pensare a un certo rapporto di causa ed effetto tra cinture e aurora: in effetti i corni della fascia esterna insistono intorno a una latitudine geomagnetica approssimativamente compresa tra $50^{\circ}$ e $60^{\circ}$; sebbene tale zona di latitudine sia alquanto più bassa di quella che definisce le zone auromali, si può tuttavia osservare che il bordo esterno della cintura di van Allen si adagia su linese di forza che raggiumgono la Terra a latitudini prossime ai $70^{\circ}$ e che, d'altra parte, la forma presunta della cintula è quella dedotta da misure di particelle piuttosto energetiche, mentre, come abbiamo visto, alquanto scarse sono le misure relative a particelle di più bassa energia; di conseguenza la 


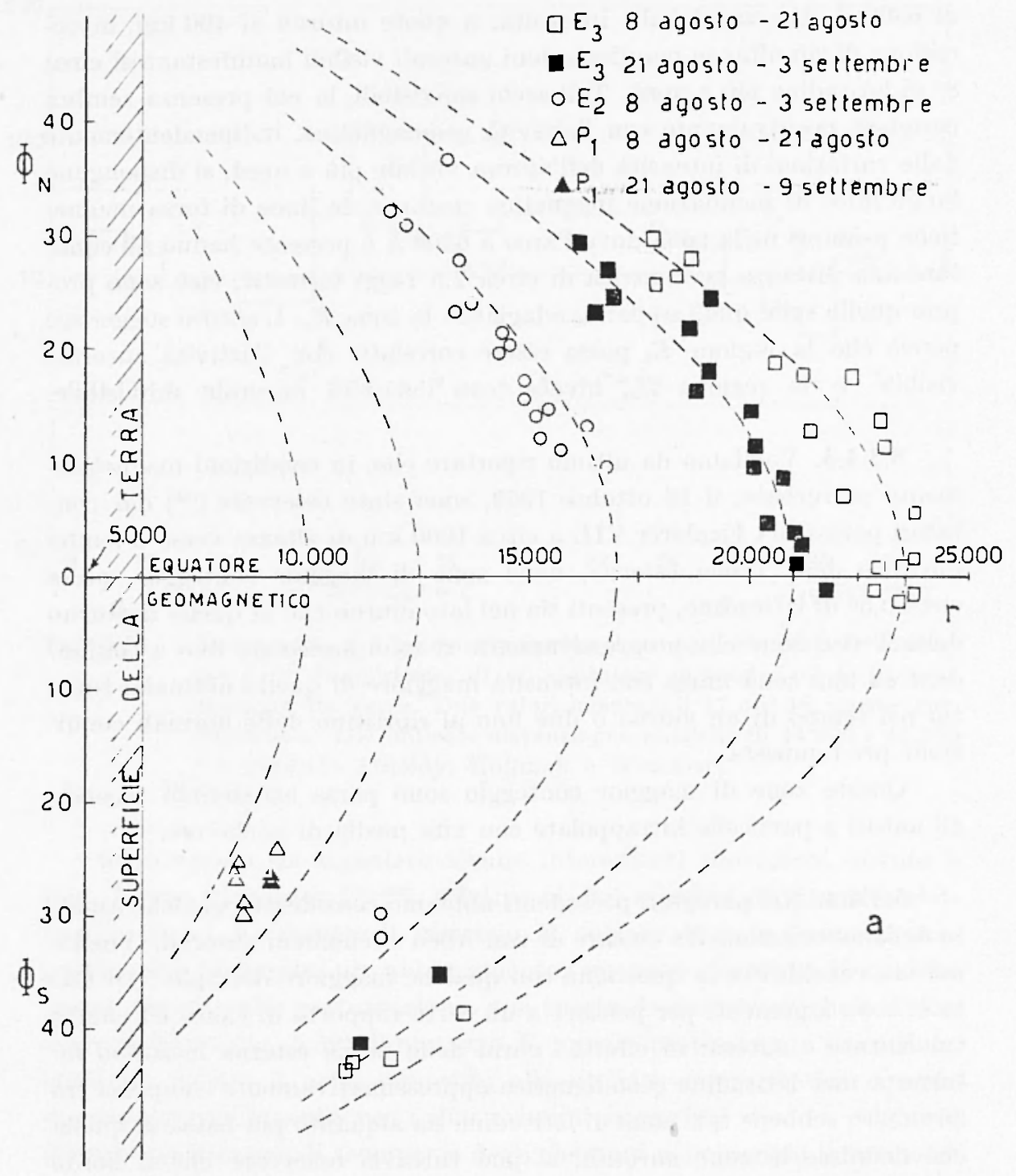

Fig. $5.32 a$ 

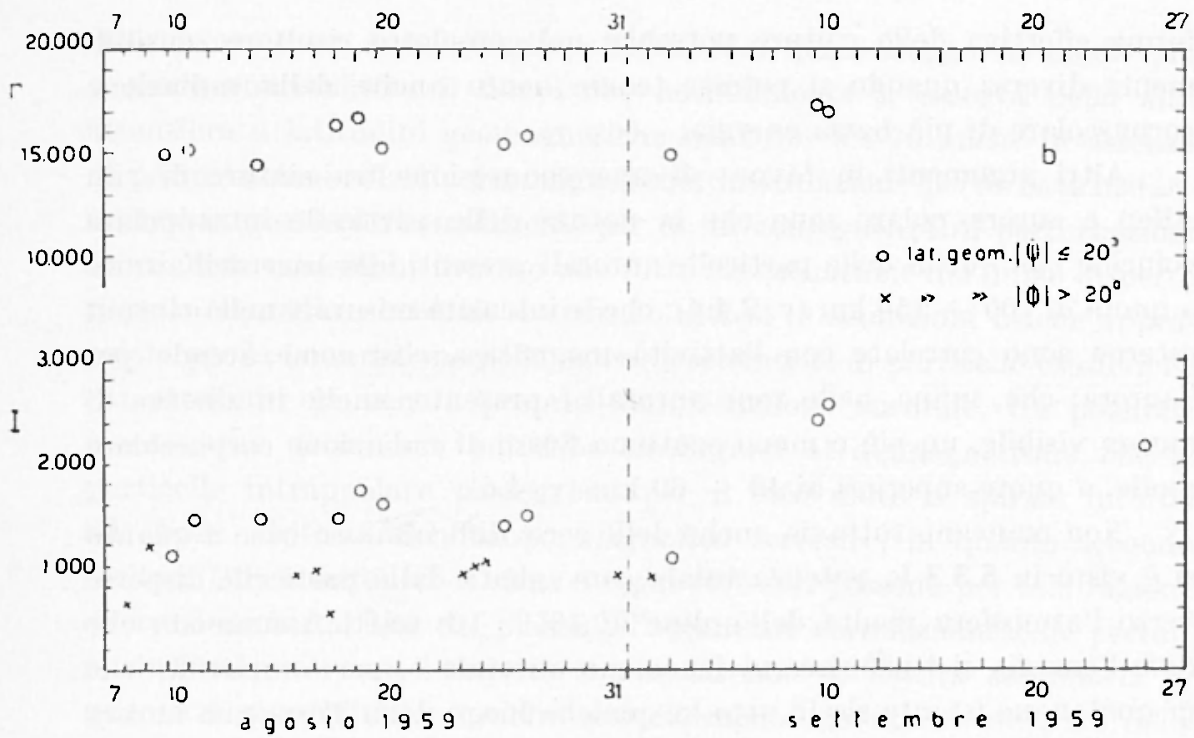

Fiø. $\tilde{\mathbf{5} .32} b$
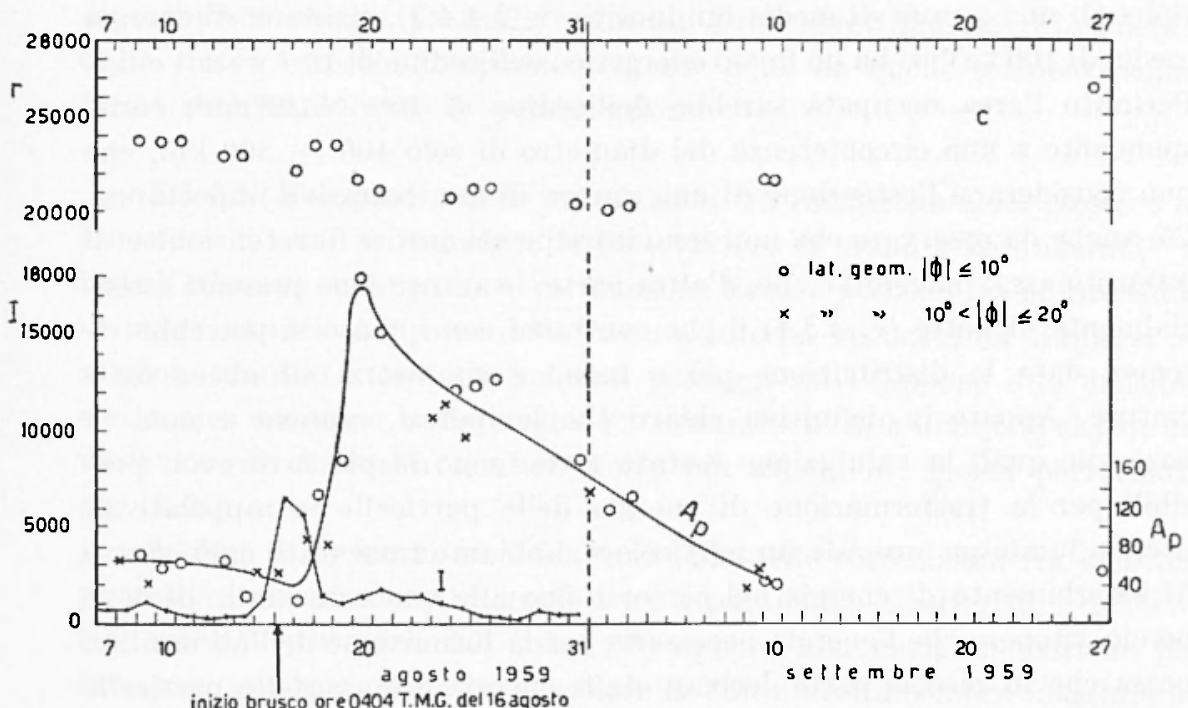

Fig. 5.32 $c-$ In fig. $a$, distanza misurata dei massimi di intensità $E, E_{3}$ e $P_{1}$; i punti neri si riferiscono a misure effettnate dopo il 19-20 agosto; le linee tratteggiate danno l'andamento delle linee di forza nell approssinazione di dipolo centrato: in fig. $b$ e $c$ anda menti temporali del massimo $I$ dell'intensità di contegrio (in impulsi/sec) nella regione $E_{2}$ e nella regione $E_{3}$ rispettivanente. della distanza neocentrica $r$ (in kin) di tali massini e (in fig. e) dellindice magnetico $A_{p}$ (secondo Fan, Mejer e Simpson). 
forma efiettiva delle cinture potrebbe nel complesso risultare semsibilmente diversa quando si potesse tenere conto anche della radiazione corpuscolare di più bassa energia.

Altri argomenti in favore di una commessione tra cinture di van Allen e aurora polare sono che la natura delle particelle intrappolate coincide con quella delle particelle auromali presenti alla base dell'auroua a quota di $100 \div 150 \mathrm{~km}$ (v. 2.4.4); che le intensità misumate nella cintura esterma sono correlate con l'attività magnetica, così come accarke per l'aurora; che, infine, nelle zone aurorali a presente, anche in assenza di aurora visibile, un più o meno continuo flusso di raddiazione corpuscolare molle, a quote superiori ai $40 \div 60 \mathrm{~km}$ (r. 4.6).

Non mancano tuttavia anche delle serie difficoltà. In base a quanto si è visto in 5.3.3 la potenza totale convogliata dalle particelle disperse verso l'atmosfera risulta dell'ordine di $10^{7} \div 10^{\mathrm{s}}$ watt. Assumendo che tutta l'energia si trasferisca al fenomeno aurorale e', per semplicità, che in qualunque istante sia in atto in qualche luogo della Terra una auroma (v. 2.2.1), si valuta facimente un limite superiore dell'area media interessata al fenomeno: con mu flusso medio di $10^{7}$ particelle $\cdot\left(\mathrm{mm}^{-2} \cdot \mathrm{sec}^{-1}\right.$, tipico di una auroma di media luminosità (v. 2.4.4.2), ciascuna di energia media di $100 \mathrm{keV}$, si ha un flusso energetico dell'ordine di $10^{-7}$ watt · 'm-2 $^{-2}$. Pertanto l'area oceupata salrebbe dell'ordine di $10^{14} \div 10^{15} \mathrm{~cm}^{2}$, corrispondente a una cinconferenza del diametro di solo $100 \div 300 \mathrm{~km}$, che juó considerarsi l'estensione di una aurora di non eceessiva importanza. Cè anche da osservare che non sono infrequenti aurore di estensione e di intensità assai maggiori e che, d'altra parte, le aurore sono presenti essenzialmente di notte (v. 2.3.1) il che contrasta con quanto si potrebbe ritenere data la distribuzione più o meno a simmetria cilindrical delle cinture. Appare in definitiva chiaro che le ipotesi, espresse e non, in base alle quali la valutazione è stata fatta, sono le più favorevoli possibili per la trasformazione di energia delle particelle intrappolate in energia luminosa auromale (in particolare, abbiamo trascumato ogni effetto di assorbimento di energia nel pereorso fino alle quote aurorali). Si deve perciò ritenere che l'energia necessaria per la formazione dell'aurora non possa che in piccola parte derivare dalla energia propria delle particelle intrappolate; si deve pensare che qualche altra più intensa sorgente di energia intervenga in modo determinante. Di ciò occorre tener conto anche nelle teorie dellorigine delle fasce di radiazione.

Uno schema ragionevole sembra quello di ritenere l'amora polare collegata alla presenza delle cinture di radiazione, non però nel senso stretto di effetto e, rispettivamente, di causa. Si può pensane la cintura 
esterna come un serbatoio di particelle dal quale sfugge in modo piì o meno continuativo il flusso che nomalmente si osserva nella alta atmosfera a latitudini geomagnetiche tra i $50^{\circ}$ e i 70 anche in assenzal di perturbazioni solari e geomagnetiche; la situazione perturbata instaurantesi in particolari condizioni, per es. in conseguenza di perturbazioni solari, determinerebbe intanto una forte accentuazione del flusso disperso rispetto a quello "nommale ", creando altresi le condizioni fisiche appropriate per la istamrazione delle cause acceleratrici di particelle esosferiche cui attribuire la vera e propria manifestazione aurorale. Un possibile mecanismo di innesco potrebbe ricollegarsi al diamanetismo che le particelle intrappolate posseggono per il loro moto a spirale intorno alle linee di forza del campo magnetico terrestre, in quanto secondo Kellogg ( $\left.{ }^{106}\right)$ a distanza di circa 8 raggi ter'estri possono per tale ragione aversi delle instabilità del plasma: l'apparente correlazione delle perturbazioni aurorali con l'attività solare sarebbe di natura secondaria in quanto a quest'ultima sarebbe semplicemente dovuto l'e innesco » delle perturbazioni.

Le considerazioni che abbiamo fatto non permettono naturalmente di escludere che le particelle aurorali siano direttamente provenienti dallo spazio esterno, indipendentemente o no da quelle incidenti sulla fascia esterna durante le perturbazioni.

5.3.4.5. Nel quadro delle correlazioni tra radiazione nella esosfera e ionosfera ci sono da segnalare le osservazioni di Sono e Fawakami (107) i quali rilevano un'apparente correlazione lineare positiva tra la intensità di raggi cosmicei al disotto della cintura interna misurata da Explorer I a quote tra 300 e $500 \mathrm{~km}$ nei suoi passaggi sul Giappone e la densità elettronica massima dello strato F2 misurata a Kokubunji (Tokyo); la variazione di intensità sembra molto più rapida nei gion'ni perturbati (fig. 5.33).

Inoltre dallo studio su scala mondiale delle correlazioni tra densità elettronica massima nello strato F2 e i parametri caratteristici dell'attivita solare per vari cicli solari $\left({ }^{108}\right)$ appare una netta indicazione in favore di una radiazione corpuscolare la cui intensità sembra massima a latitudini geomagnetiche di $50^{\circ}-60^{\circ}$ che sono, come si è visto, quelle verso le quali insistono i "corni " della cintura esterna. L'energia delle singole particelle costituenti la detta radiazione corpuscolare cadrebbe nella regione dei $\mathrm{keV}$. Se una radiazione corpuscolare è effettivamente presente nella ionosfera si dovrebbe ritenere anche presente un effetto per cosi dire meteorologico: cioè un riscaldamento dell'atmosfera alle 
quote ionosferiche là dove il flusso di energia ì praticamente continuo nel tempo; questo riscaldamento dovrebbe a sua volta provocare un innalzamento delle superfici isobariche rispetto alle corrispondenti quote alle basse a alle molto alte latitudini.

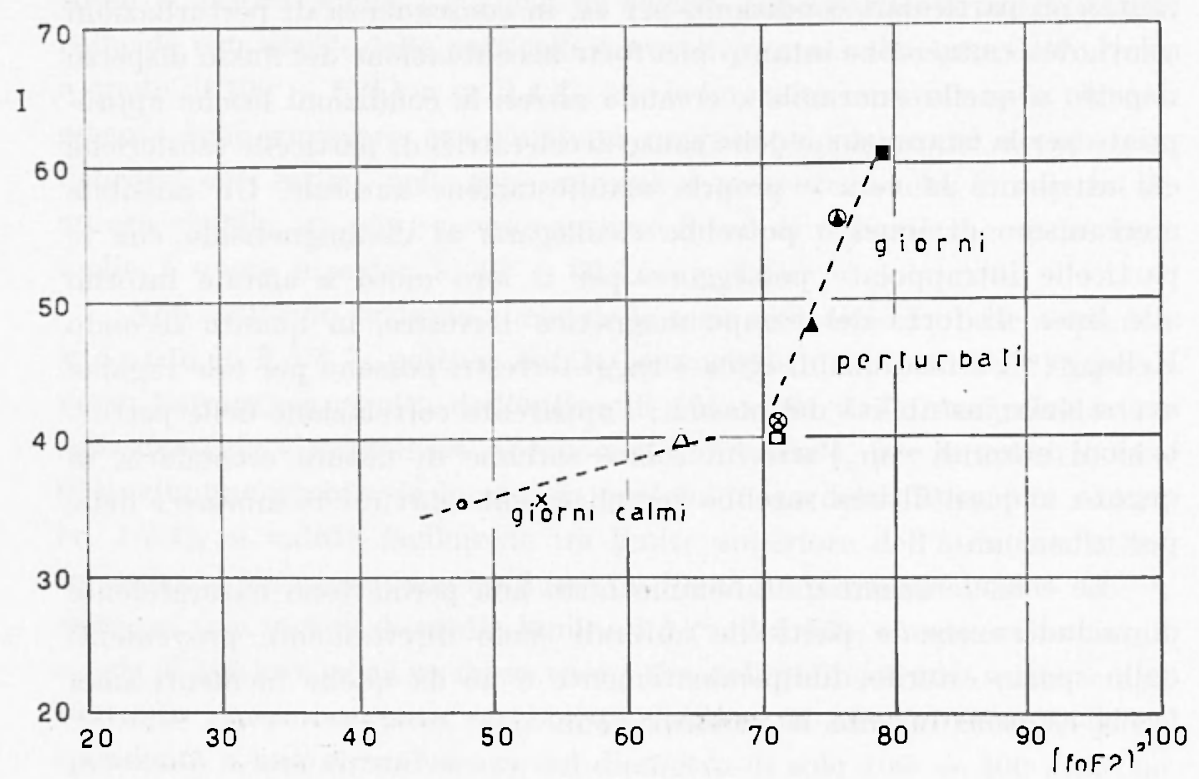

Fig. 5.33 - $I$, intensità di contegrio in impulsi/sec: sulle ascisse. il quadrato della frequenza critica dello strato $F_{2}$ misurata alle ore 20 di tempo locale (ore 10 T.M.G.); $\mathrm{i}$ diversi pmuti si riferiscono a giorni diversi nel periodo $1-11$ fehbraio 1958 (secondo dono e Kawakani).

In effetti misure di temperatura condotte su razzi dànno chiara evidenza, a parità di quote, di temperatura notevolmente più alta alle latitudini aurorali ehe a latitudini inferiori: ai $\sim 1000$ oK misurati, intorno a $150 \mathrm{~km}$ dal suolo, a White Sands (lat. geomagn. $41^{\circ} \mathrm{N}$ ) corrispondono da 1500 a 2000 oK a Churchill (lat. geomagn. 690 N). Una stima grossolana del riscaldamento prodotto nelle zone aurorali alle quote ionosferiche dal flusso energetico convogliato da particelle sfuggenti dalle cinture di van Allen porta a valori $\left.{ }^{(109}\right)$ intorno ai 2000 oli; in più la distribuzione in altezza della energia termica eeduta all'atmosfera dal flusso di elettroni presenta un massimo assai netto intorno ai $100 \mathrm{~km}$ dal suolo così come aceade per la massima frequenza di occorrenzal delle aurore. 
5.3.5. Origine delle cinture di radiazione. -.. Prima di esaminare con qualche dettaglio il problema dell'origine delle cinture di ran Allen, vogliamo costruire un quadro pì̀ o meno approssimativo della dinamica delle particelle intrappolate. Queste particelle sono soggette a vari tipi di moto:

a) moto circolare intorno alle linee di forza del campo magnetico con periodo $\tau_{1}$ proporzionale alla terza potenza della distanza $L$ definita in 5.3.2.1;

b) oscillazione lungo le linee di forza, tra opposti punti di riflessione, con periodo $\tau_{2}$ proporzionale a $L$;

c) deriva in longitudine determinata da due cause: la prima dall'efietto combinato del campo geomagnetico e della forza rentrifuga cui le particelle oscillanti lungo le linee di forza magnetica sono soggette per la curvatura di queste; la seconda dalleffetto rombinato del campo magnetico e del suo gradiente radiale. La velocità di deriva è orientata, per ambedue le cause, verso est per particelle negative e verso ovest per particelle positive. Il periodo $\tau_{3}$ del risultante moto di rotazione intorno alla Terra è inversamente proporzionale a $L$.

La tabella 5.4 riporta alcuni valori di $\tau_{1}, \tau_{2}$ e $\tau_{3}$, in sec., calcolati per $L=2$ raggi terrestri e per particelle aventi, all'equatore, angolo di inclinazione prossimo a $90^{\circ}$.

Tabella 5.4

\begin{tabular}{|c|c|c|c|c|c|}
\hline \multicolumn{3}{|c|}{ Elettroni } & \multicolumn{3}{|c|}{ Protoni } \\
\hline & $10 \mathrm{ker}$ & $100 \mathrm{ker}$ & $1 \mathrm{MeV}$ & 10 Mer & $100 \mathrm{MeY}$ \\
\hline$r_{1}$ & $9,4 \cdot 10^{-6}$ & $1,1 \cdot 10^{-5}$ & $1.74 \cdot 10^{-2}$ & $1.76 \cdot 10^{-2}$ & $1.91 \cdot 10^{-2}$ \\
\hline$\tau_{2}$ & $6,4 \cdot 10^{-1}$ & $2,3 \cdot 10^{-1}$ & 2,7 & $8.6 \cdot 10^{-1}$ & $2.9 \cdot 10^{-1}$ \\
\hline$\tau_{3}$ & $1,4 \cdot 10^{5}$ & $1,4 \cdot 10^{4}$ & $1,3 \cdot 10^{3}$ & $1.4 \cdot 10^{2}$ & $1,4 \cdot 10^{1}$ \\
\hline
\end{tabular}

Ai moti periodici sopra considerati si aggiungono la deriva e la diffusione lungo la verticale dovuta al gradiente longitudinale de] campo nonchè, più in generale, i moti dovuti a disomogeneità del campo rispetto alla approssimazione di dipolo o impresse dall'esterno al campo "statico". 
In conseguenza di tali moti, se si assume, indipendentemente dalle cause, l'esistenza di un flusso di particelle penetrante nella esosfera a qualche raggio terrestre di distanza si possono prevedere: ma dispersione di parte di esse, incanalate lungo le linee di forza magnetiche fin verso la ionosfera e le quote auromali; una progressiva diffusione verticale verso la bassa atmosfera e un moto di insieme in senso ovest est per gli elettroni, est ovest per i protoni; la produzione di effetti magnetici.

Non entriamo nello studio dettagliato dei processi fisici di interazione delle particelle intrappolate con le particelle che costituiscono l'esosfera; ricordiamo soltanto che parecchi antori hamno calcolato per numerosi processi la vita media di particelle intrappolate. I risultati sono notevolmente condizionati dai modelli di esosfera adottati; d'altra parte la grande incertezza e la grande difficoltà di identificazione della natura delle particelle rendono problematico allo stato attuale il confronto tra risultati dei calcoli e dati sperimentali.

5.3.5.1. Campo magnetico delle particelle intrappolate. - I moti regolari delle particelle intrappolate, di cui sopra si è fatto cemno, hamno tra le altre la conseguenza di generare un cumpo magnetico che si sovappone a quello geomagnetico.

Nel moto a spirale intorno alle linee di forza geomagnetiche ogni particella dà origine a un momento di dipolo di valore

$$
M=W_{\perp} / B
$$

ove $B$ è l'induzione magnetica, $W_{\perp}$ la frazione di energia cinetica dovuta alla componente di velocità nomale al campo geomagnetico (indicheremo più avanti con $W_{\|}$l'analoga frazione dovuta alla componente parallela).

Nel moto di oscillazione lungo le linee di forza magnetiche tra opposti punti di riflessione il campo magnetico complessivo risulta praticamente mullo sia per l'interferenza degli eftetti di particelle oscillanti in rersi opposti sia per la simmetria intorno all'asse del dipolo geomagnetico.

Nel moto di deriva longitudinale, infine, il campo è quello prodotto da un dipolo situato nel centro di simmetria della traiettoria circolare della singola particella intorno alla terra, di momento di valore

$$
M=\frac{3}{2} \frac{W \perp}{B}
$$

e

$$
M=2 \frac{W_{\|}}{B}
$$


rispettivamente per l'effetto collegato alla forza centrifuga e al gradiente radiale del campo. Mentre rinviamo al cap. 6 per una discussione di questi effetti magnetici e sulla loro importanza nella teoria delle tempeste magnetiche, ri limitiamo qui ad osservare che in ricinanza della Terra il campo dovuto al momento [5.6] ì concorde a quello geomagnetico; quello dovuto invece ai dipoli [5. $]$ e [5.8] ì di senso opposto.

5.3.5.2. Origine della cintura interna - Vari autori (106, 110, 111) hanno attribuito fin dall'inizio la cintura al decadimento radioattivo dei neutroni di albedo prodotti in alta quota nelle interazioni nucleari della radiazione cosmica primaria con le particelle dell'atmosfera.

La probabilità di tali interazioni è naturalmente apprezzabile solo a quote inferiori a qualche decina di $\mathrm{km}$, cosicehé si può senzaltro trascurare l'effetto di albedo per le particelle cariche che si producono in tali interazioni: ciò in quanto il campo geomagnetico è abbastanza intenso da incurvare sensibilmente le tratettorie fino a farle rientrare nell'atmosfera. I neutroni, invece, non interagendo con il campo geomagnetico, possono più facilmente disperdersi nell'esosfera dove poi, per disintegrazione, damno origine a protoni ed elettroni; questi, infine, per il fatto di essere iniettati in regioni dove la densità di materia è ormai molto bassa, possono rimanere intrappolati dal campo mannetico terrestre con vita media anche molto lunga.

Stime dellordine di grandezza del flusso di neutroni di albedo damno $\left(^{112}\right)$ valori di qualche unità di $0,1 \mathrm{~cm}^{-2} \cdot \mathrm{sec}^{-1}$ e comunque, per ogni determinata quota, crescenti dall'equatore geomagnetion ai poli per un fattore di qualche unità (fig. $5.34 a, b$ ).

Dalla disintegrazione prendono origine elettroni di energia non superiore a $782 \mathrm{ker}$ e protoni di rinculo di bassa energia; lo spettro caleolato degli elettroni intrappolati presenta, secondo Kellogo $\left({ }^{113}\right)$, un massimo tra 400 e 500 keV (fig. 5.35, eurva $a$ ); dallo spettro si deduce il flusso per moltiplicazione per un fattore $v$ velocità (curva $b$ ); ne consegue che il flusso calcolato ha il suo massimo spostato leggermente verso energie più alte.

Fella tabella 5.5 riportiano alcuni valori di densità e di flussi di particelle, pure calcolate da Kellogg in prossimita del piano equatoriale a distanza di qualche raggio terrestre, nell'ipotesi che l'unicar causal di scomparsa delle particelle intrappolate sia lo scattering assumendo vite medie di $3 \cdot 10^{9}$ e di $10^{12}$ sec, rispettivamente per alettroni e protoni. Di fatto, come si è già accennato, le effettive vite medie delle particelle intrappolate risultano senz'altro di qualehe ordine di grandezza inferiori 


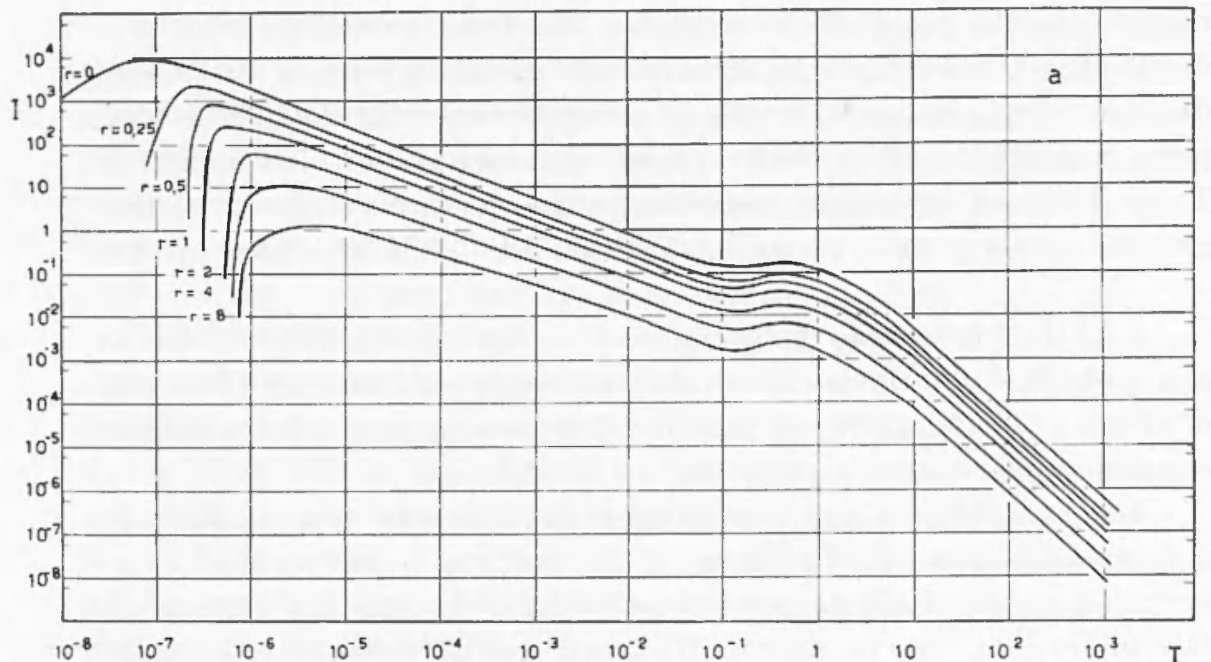

Fig. 5.3t - In figg. a, flusso differenziale $I$ di neutroni (in nentroni $\cdot \mathrm{em}^{-2} \cdot \mathrm{sec}^{-1}$. Me $\mathrm{V}^{-1}$ ) nellatmosfera terrestre, calcolato sul piano equatoriale geomagnetico: le distanze $r$ sono indirate in raggi terrestri a partire dal limite estre mo della atmosfera, fissato a $\sim 100 \mathrm{~km}$ dal suolo; T', energia cinetica in $\mathrm{MeV}$; in fig. $b$ linee di uguale densitì di decadimento $\beta$ (in $10^{-11}$ decadimenti $\cdot\left(\mathrm{cm}^{-3}\right.$. sec $^{-1}$ in 111 piano meridiano geomagnetico; le distanze reocentrichle sono in raggi terrestri; $\Phi_{\Lambda}, \quad \Phi_{S}$ latitudine geomagnetica nord e sud (secondo Hess, Canfield e Lingenfelter).

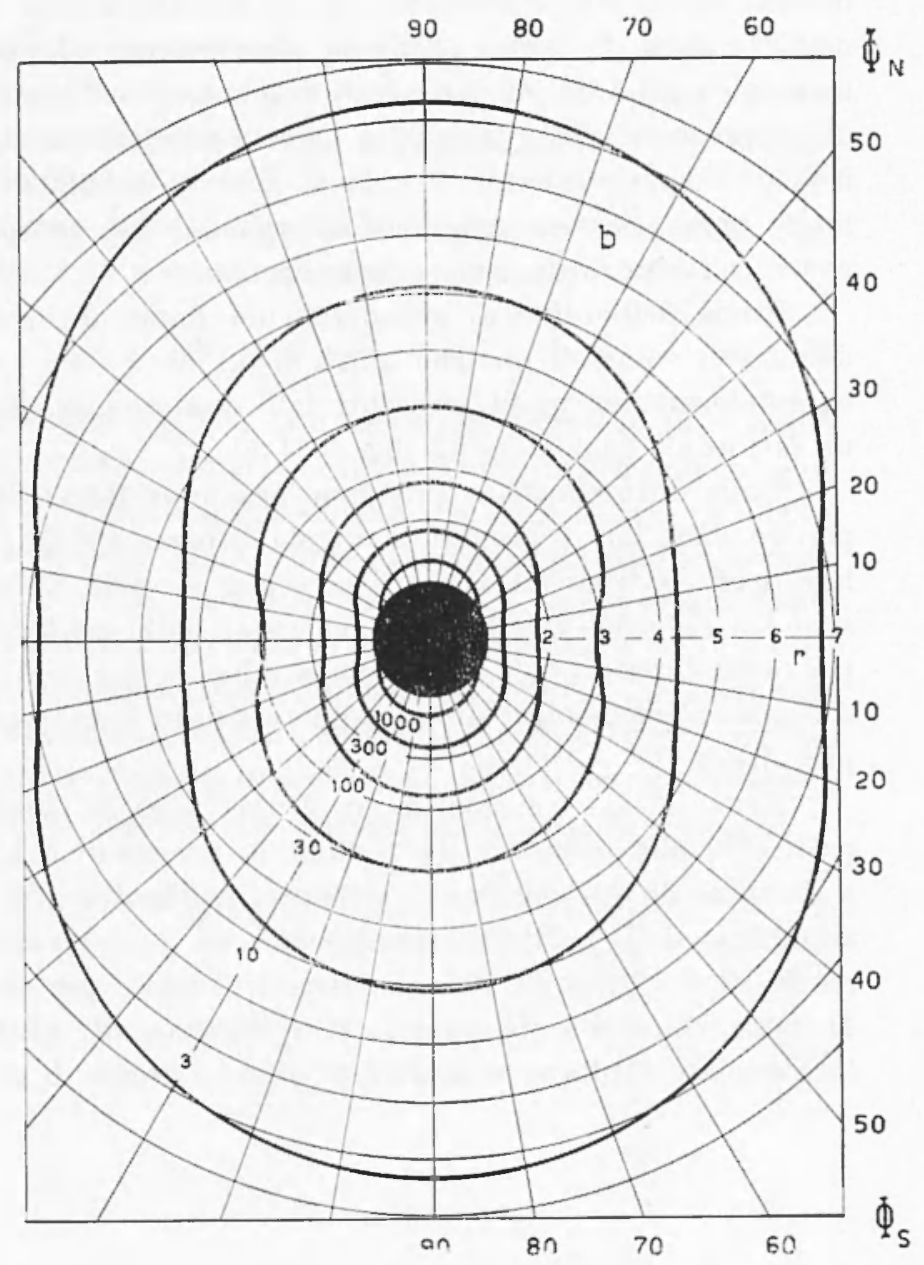


a quelle assunte, evidentemente per' l'effetto di altri processi di scomparsa oltre lo scettering: i valori riportati nella tabella sono quindi superiori, per vari ordini di grandezza, a quelli reali.

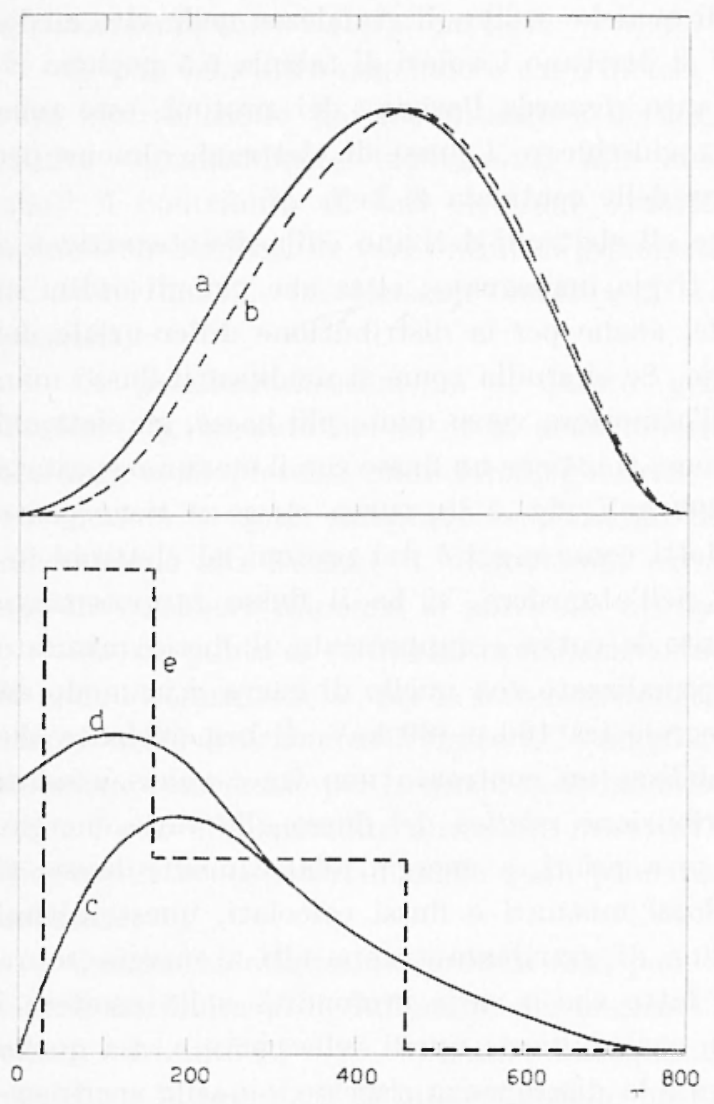

Fig. ธ.35-sulle ordinate, unitì arbitrarie; la curva a dì nuo spettro differenziale, le altre curve damno flussi differenziali; $T$, energia cimetica in ker (se. condo Kelloggr).

Tabella 5.5

\begin{tabular}{|c|c|c|c|}
\hline & Elettroni & $\begin{array}{l}\text { Protoni } \\
\text { di } \overline{5} \text { MeT }\end{array}$ & $\begin{array}{l}\text { Protoni } \\
\text { di 50 MeV }\end{array}$ \\
\hline Densitia di particelle (in $c^{-3}$ ) . . & $6 \cdot 10^{-3}$ & $2 \cdot 10^{-3}$ & $10^{-3}$ \\
\hline Flusso di particelle (in $\mathrm{cm}^{-2} \cdot \mathrm{sec}^{-1}$ ) & $2 \cdot 10^{8}$ & $6 \cdot 10^{6}$ & $10^{7}$ \\
\hline Flusso di energia in (erg. $\left.\mathrm{em}^{-2} \cdot \mathrm{sec}^{-1}\right)$ & 100 & 50 & 800 \\
\hline
\end{tabular}


Se si confrontano i valori calcolati di tabella 5.5. con quelli misurati di tabella 5.2 si nota che il flusso di elettroni e i flussi energetici calcolati concordano nellordine di grandezza ma il flusso di protoni energetici è $10^{3} \div 10^{1}$ volte maggiore di quello misurato. E chiaro allora che conseguenza della riduzione di qualche ordine di grandezza nelle vite medie è che, se le ipotesi da cui si derivano i valori di tabella 5.5) possono ritenersi ancottabili per quanto riguarda l'origine dei protoni, esse sono decisamente insufficienti a giustificare i flussi di elettroni, almeno per quelli di energie dell'ordine delle centinala di keV.

In effetti, l’ipotesi che gli elettroni derivino dalla disintegrazione $\beta$ dei neutroni di albedo si rivela inaleguata, oltre che per gli ordini di grandezza del flusso totale, anche per la distribuzione differenziale del flusso tra le diverse energie. Se si studia come si modifica il flusso man mano che, penetrando nell'atmosfera verso quote più basse, gli elettroni perdono energia per collisione, si ottiene un flusso con il massimo spostato verso energie intorno a $200 \mathrm{keV}$ (fig. 5.35, curva e); se si tiene conto anche degli elettroni prodotti come raggi $\delta$ dai protoni ed elettroni intrappolati che penetrano nell'atmosfera, si ha il flusso rappresentato dalla curva d; per confronto la curva $e$ rappresenta il flusso misurato da Holly e Johnson ( $\left.{ }^{84}\right)$ nommalizzato con quello di curva d in modo da dare un uguale flusso integrale tra 160 a $460 \mathrm{keV}$. E ben evidente che con tale procedura si stabilisce un confronto non tra i valori assoluti dei flussi, ma tra la distribuzione relativa del flusso alle varie chergie. Risulta chiarmente che se a priori, a energie relativamente basse, si postula un aceordo tra flussi misurati e flussi calcolati, questi ultimi sono invere circa un ordine di grandezzal troppo alti a energia sopla i 460 keV. In più, per il fatto che a varie profondità nella esosfera i flussi calcolati variano con continuità da quelli delle curve $e$, d a quello di curva b, tanto maggiore è la discrepanza rispetto a quello sperimentale osservando che Ie misure di Holly e Johnson si riferiscono a quote sotto la cintura interna ma tuttavia molto vicino all essa: l'ambamento teorico del flusso da confrontarsi con le determinazioni sperimentali è pertanto assai più prossimo a quello di curca $b$ che a quello delle curve $c$, d. In definitiva il contributo relativo calcolato dello encregie più alte rispetto a quelle più basse puó facilmente risultare di $3 \div 4$ ordini di grandizza troppo elevato.

Ancora grosse discrepanze nella forma dei flussi (e degli spettri) differenziali misurati e calcolati vengono segnalate da vari autori (114.115): in particolare, prendendo in considerazione anche la perdita di energia nell'attraversamento della materia esosferica, Lenchek. Singer e Went- 
worth (14) trovano che di fronte a un certo accordo quantitativo tra valori misurati a valori calcolati dei flussi assoluti a energie superiori a circa $400 \mathrm{keV}$, questi ultimi risultano invece $2 \div 3$ ordini di grandezza inferiori a energie sotto i $100 \div 200 \mathrm{keV}$. Ie discrepanze si esaltano ulteriormente sotto i $100 \div 50 \mathrm{keT}$.

Si può senz'altro conchudere che l'ipotesi che grli elettroni della cintuma interna siano dovuti soltanto a decadimento beta dei neutroni risulta essenzialmente inaleguata. Alle energie più basse poi, alle quali il contributo di tali elettroni è assolutamente trascurabile, si richiedono sorgenti di vari ordini di grandezza più intense. Potrebbe ad es. arersi qualcho mecranismo "locale" di accelerazione di elettroni già presenti.

Un possibile mecumismo di questo genere viene suggerito dat Dessler (116), secondo cui al di là della ionosfera fino a qualche raggrio terrestre sono presenti onde idromagnetiche di ampiezza dellordine di $10^{3}$ gramma le quali sarebbero responsabili della accelerazione, con un mecoanismo alla Fermi $\left({ }^{117}\right)$, di particelle cariche "locali " dei due segni che diverrebbero appunto le particelle intrappolate nelle cinture.

Questo punto di vista è tuttavia contrastato da Singer (110) in quanto le onde idromagnetiche, per la loro caratteristica di propagarsi principalmente nella direzione delle linee del campo magnetice, e assai poco in direzione trasversale per la minore conducibilità trasversa, risulterebbero efficaci solo alle latitudini aurorali e molto di meno o affatto alle latitudini medie ed equatoriali, nelle quali principalmente le cinture sono lo$\because$ alizzate.

Quanto all'origine dei protoni essa puó, almeno in parte notevole, essere attribuita al decadimento dei nentroni di albedo; oltre quelli già (itati, vari autori $\left({ }^{118,}{ }^{119,}{ }^{120}\right)$ hamno dettagliatamente stucliato le caratteristiche e le proprieti di tale radiazione protonica. Tuttavia ralgono le riserve espresse in 5.3.4.1 circa la presenza di protoni provenienti da altre sorgenti; in particolare protoni di origine solare.

5.3.5.3. Origine della cintura esterna. - Nella fascia esterna, come si è detto, le particelle rivelate sono essenzialmente elettroni di energia relativamente bassa. Parecchi autori hamno suggerito che questi elettroni siano di origine extraterrestre, in particolare di origine solare: essi, emessi dalla sorgente in modo saltuario o con continuità, non potendo penetrare per la loro bassa energia nella zona di più intenso campo magnetico terrestre, resterebbero intrappolati lungo le sue linee di forza esterne. 
Questa verluta si presta a varie critiche; in particolare Dessler e Karplus (221) mettono in evidenza varie difficoltà: il flusso misurato ap)pare diminuito durante la fase attiva della perturbazione geomagnetica, cioè proprio nella lase in cui ei si clovebbe aspettare l'entrata delle particelle solari; un aumento di parechi ordini di grandezza del flusso si verifica invece nella fase di recupero della tempesta magnetica, proprio cioc quamdo il ripristino del eampo geomagnetico a i normali valori « quieti " dovrebbe far ritenere assai improbabile l'entrata di particelle provenienti dal Sole; le rapirle variazioni del flusso di particelle nel corso di perturbazioni geonagnetiche sembrano un indice di molto breve vita media delle particelle stesse, mentre questa a giulicare dalla stabilità della cintula dumante i periodi magneticamente calmi sembrerebbe essere parecrhio più lunga.

Secomelo gli Autori la cansa delle vamiazioni nella cintura sta piuttosto che in variazioni "primarie " di flusso, nelle variazioni del campo magnetico, nel senso che queste provocano variazioni di energia delle particelle già presenti con conseguente valiazione della loro distribuzione spettrales, tanto più rilevante quanto maggiore è la ripidità dello spettro energetico: l'effetto di soglia di enereria negli strumenti rivelatori simulerebbe cosi variazioni di intensità totale. Altra causa di variazioni può ricercarsi nello spostamento "lento" (lento rispetto al periorlo $\tau_{2}$ del moto a spirale delle particelle) delle linee di forza manetiche che provorherebbe uno spostamento di insieme delle particelle ad esse "agganciate" e, con questo, di nuovo una variazione più o meno rilevante del flusso in un determinato punto dello spazio.

Gli Autori propendono per l'attribuzione anche dell'origine della cintura esterna al decalimento $\beta$ di neutroni: di questi quelli lenti, assai numerosi, darebbero luogo alla formazione della cintura esterna mentre quelli veloci, sarebbero responsabili della formazione della cintura interna.

Hess a Killeen (122) sturliano ron un certo dettaglio il rontributo degli elettroni di decadimento e concludono che mentre ad enelgie sopra qualche centinaio di keV i flussi sperimentali presentano un certo aceordo con quelli caleolati, non così arviene per le energie più basse; gli Autori ritengono commque che per energie superiori ai $50 \mathrm{keV}$ una significativa frazione degli elettroni possa attribuirsi al deculimento $\beta$ dei neutroni di alberlo.

Un'interpretazione completa dei risultati sperimentali richiedle, come grà riscontrato nella fascia interna, la presenza di altre sorgenti di iniezione di elettroni. Una conclusione analoga viene suggerita (123) dallo 
studio dell'andamento dei flussi misurati lungo una stessa linea di forza da Explorer VI.

Per ciò che concerne i protoni, la loro rapida scomparsa al crescere della distanza da Terra viene attribuita da Singer (124) a una rapida diminuzione della vita media delle particelle intrappolate più energetiche, per il venir meno (breakdown), al crescere della distanza da Terra e rell'energia delle particelle, della condizione di "arliabaticità ", cioè di lentissima variazione dell'angolo di inclinazione da una oscillazione all'altra tra successivi punti di riflessione. Nelle zone più esterne, ciò nella eintura esterna, resterebbero intrappolate solo le particelle meno energetiche per le quali la suddetta condizione di adiabaticità resterebbe valida: secondo l'Autore già a energie di $70 \mathrm{MeV}$ i protoni si troverebbero in rondizioni di instabilità. Altri autori, tuttavia, ritengono in contrasto con Singer che ancora a distanze di due raggi terrestri i protoni di 500 HeV possano restare intrappolati in modo stabile.

Welch e Whitaker (125) a loro volta pensano che, sebbene sia possibile un vero e proprio intrappolamento di protoni energetici su orbite stabili, vi sarebbero tuttavia effetti di onde idromagnetiche che provocherebbero variazioni del momento magnetico dei protoni spilalizzanti intorno alle linee del campo magnetico: conseguenza di ciò sarebbe un continuo susseguirsi di spostamenti a caso (random wall) dei loro punti di riflessione, il che in definitiva farovirebbe la scomparsa dei protoni nella atmosfera densa.

Una particolare questione, che resta per il momento almeno esclusivamente sul piano teorico per le limitazioni imposte dai rivelatori, riguarda i protoni di energie dell'ordine dei $\mathrm{keV}$ fino al $\mathrm{MeV}$, in particolare i protoni di origine solare di energie di $20 \div 100$ keV (i quali percorrerebbero la distanza Sole-Terra in circa un giorno e sarebbero responsabili delle temepeste magnetiche): secondo Stuart ( $\left.{ }^{126}\right)$ protoni in tale intervallo di energia non arrebbero grande rilevanza nella alimentazione della cintura esterna in quanto, essendo la loro velocità, dell'ordine di $1000 \mathrm{~km} / \mathrm{sec}$, assai prossima a quella periferica dellelettrone nell'atomo di idrogeno, risulta assai elevata la sezione d'urto per il processo di scambio di carica tra protoni a atomi di idrogeno presenti nella esosfera. Tenuto conto della concentrazione di atomi neutri di idrogeno a distanze di qualche raggio terrestre $\left(10^{4} \cdot \mathrm{cm}^{-3}\right.$ e $10^{2} \mathrm{~cm}^{-3}$ rispettivamente a 3 a a 6 raggi) i protoni resterebbero intrappolati con vite medie di solo qualche ora e di qualche centinaio di ore, rispettivimente. Analoghi risultati sono stati anche ottenuti da Tiemohn $\left({ }^{127}\right)$.

Secondo Jastrow ${ }^{128}$ ) invece, questi protoni potrebbero addirittura. dare origine alla fascia esterna nel senso che, provenendo dall'infinito 
su certe particolari orbite avvolgentisi più volte intorno alla Terra, essi potrebbero essere trasferiti per diffusione coulombiana dalla situazione di particelle "quasi intrappolate " a quella di particelle intrappolate; assumendo valori lagionevoli per le grandezze fisiche interessate, Jastrow valuta in $10^{24}$ protoni. sece-1 l'afflusso di protoni nella cintura esterna secondo tale meccanismo. Una volta intrappolati, i protoni equipartirebbero la loro energia con gli elettroni "termici " presenti che risulterebbero cosi accelemati, dando origine alla componente elettronica della fascia estema. Ton mancano tuttavia difficoltà al meceanismo di iniezione dei protoni dovute al fatto che la penetrazione del campo magnetico nel fascio di particelle incidenti non sembra possa essere sufficientemente rapida.

In ogni caso l'importanza teorica delle particelle di energia contenuta in questa limitata regione spettrale sta nel fatto che effettiramente la loro presenza è essenziale per la spiegazione della fenomenologia delle tempeste magnetiche e delle aurore polari.

5.3.5.4. Un aspetto particolamente interessante della fenomenologia delle fasce di radiazione è l'esistenza del minimo di intensità che separa nettamente quella esterna da quella interna, centrato a distanze geocentriche di circal due raggi terrestri (all'equatore), nonché la presenza di un minimo secondario che separa nella fascia estema le due regioni $E_{2}$ e $E_{3}$.

Una delle prime ipotesi avanzate è che le due cinture, ambedue aventi la stessa origine, per es. solare, risultino separate ${ }^{(29)}$ ) per la presenza di instabilità del campo magnetico terrestre a distanza dellordine dei due raggi terrestri; questa ipotesi tuttavia, allo stato delle conoseenze, appare poco probabile se si tiene conto della sostanziale "stati(ità " del campo magnetico terrestre a distanza di $2 \div 3$ raggi terrestiri (v. 5.1.2).

Singrer (124) attribuisce invece la separazione a un più o meno brusco venir meno della condizione di adiabaticità delle particelle più energetiche: la rapida scomparsa di queste deteminerebbe appunto la diminuzione del flusso totale misurato; poiché le particelle più energetiche sono i protoni della cintura interna, si verrebbe a determinare oltre il minimo una distribuzione di elettroni di energia piuttosto bassa, come appunto appare nella fascia esterna.

En meccanismo del tutto diverso, inizialmente proposto da Dessler $\left({ }^{130}\right)$ in modo più o meno qualitativo per spiegare la presenza del minimo tra fascia interna e fascia esterna, è stato poi consirlerato 
da Dessler e Karplus (221) per interpretare la depressione di intensità che dà origine, nella fascia esterna, alle due regrioni $J_{2}, E_{4}$. Secondo le idee degli Autori, la depressione sarebbe collegata alla presenza della regrione di campo geomagnetico sensibilmente meno intenso del campo di dipolo, nota come anomalia di Città del Capo, la quale si estende fino a parecchie migliaia di km di quota, cosicché anche alle quote di riflessione delle particelle intrappolate agganciate alle linee di forza magnetiche da essa uscenti l'intensità del campo geomagnetico risulta più bassa di quanto c.i si aspetterebbe; di conseguenza i punti di riflessione subiscono un abbassamento assai sensibile (fig. 5.36) con un corrispondente aumento dell'assorbimento di particelle intrappolate nell'atmosfera più densa. La diminuzione "locale " delle intensità si propagherebbe poi, per il movimento di deriva longitudinale delle particelle intrappolate, a tutte le particelle che vengono a trovarsi man mano agganciate alle linee di forza passanti per la regione anomala di Città del Capo; in altri termini l'effetto di accresciuto assorbimento o, se si vuole, la diminuzione di vita needia, darebbe origine a una regione di minimo a simmetria più o meno circolare intorno alla Terra. Gli effetti di questa anomalia sono stati calcolati da Dessler e Farplus in base alla citata analisi del campo geomagnetico ron 48 roefficienti di Gauss dovuta a Vestine a Sibley (1) (fig. 5.36 a) e da Cladis e Dessler $\left({ }^{131}\right)$ in base all'analisi del campo con 512 coefficienti fatta da Jensen, Muray e Welch (132) (fig. 5.36 b).

Nellipotesi grià considerata che anche la fascia esterna fosse doruta a decadimento di neutroni di albedo, l'effetto prodotto dall'anomalia di Città del Capo consisterebbe in una diminuzione del flusso a circa il 10\% di quello prevedibile se il campo geomagnetico non fosse anomalo.

La presenza del minimo tra le regrioni $E_{2}$ er $E_{3}$ viene da Dessler e Karplus considerata come prova che la cintura esterna, o almeno la regione $E_{2}$, non sia di origine solare: infatti la effeacia della anomalia di Città del Capo è subordinata alla condizione che gli elettroni debbano avere punti di riflessione sufficientemente bassi perché il meccanismo di assorbimento nella atmosfera più densa sia efficace: ciò implica una sorgente di iniezione di elettroni soprattutto localizzata a quote piuttosto basse, proprio come arviene per gli elettroni di decadimento dei nentroni di albedo (v. fig. 5. $3+b$ ).

La intepretazione del minimo tra $E_{2}$ ed $E_{3}$ proposta da Dessler e Karplus va tuttavia incontro a gravi critiche, in quanto le particelle i cui punti di riflessione scendono nella regione anomala di Città del Capo a quote sotto i $1300 \mathrm{~km}$ dal suolo, alle quali l'atmosfera comincia ad 
essere sufficientemente densa, non appationo provenienti, nel loro moto di deriva longitudinale, dalla regione nella quale secondo i dati sperimentali è stato localizzato il minimo di intensità tra le regioni $E_{2}$ ad $E_{3}$; le distanze geocentriche tra le quali le particelle intrappolate debbono essere comprese per passare, nel loro moto di deriva, attrarerso l'anomalia di Citta del Capo, risultano comprese, a seconda della longitudine, tra i

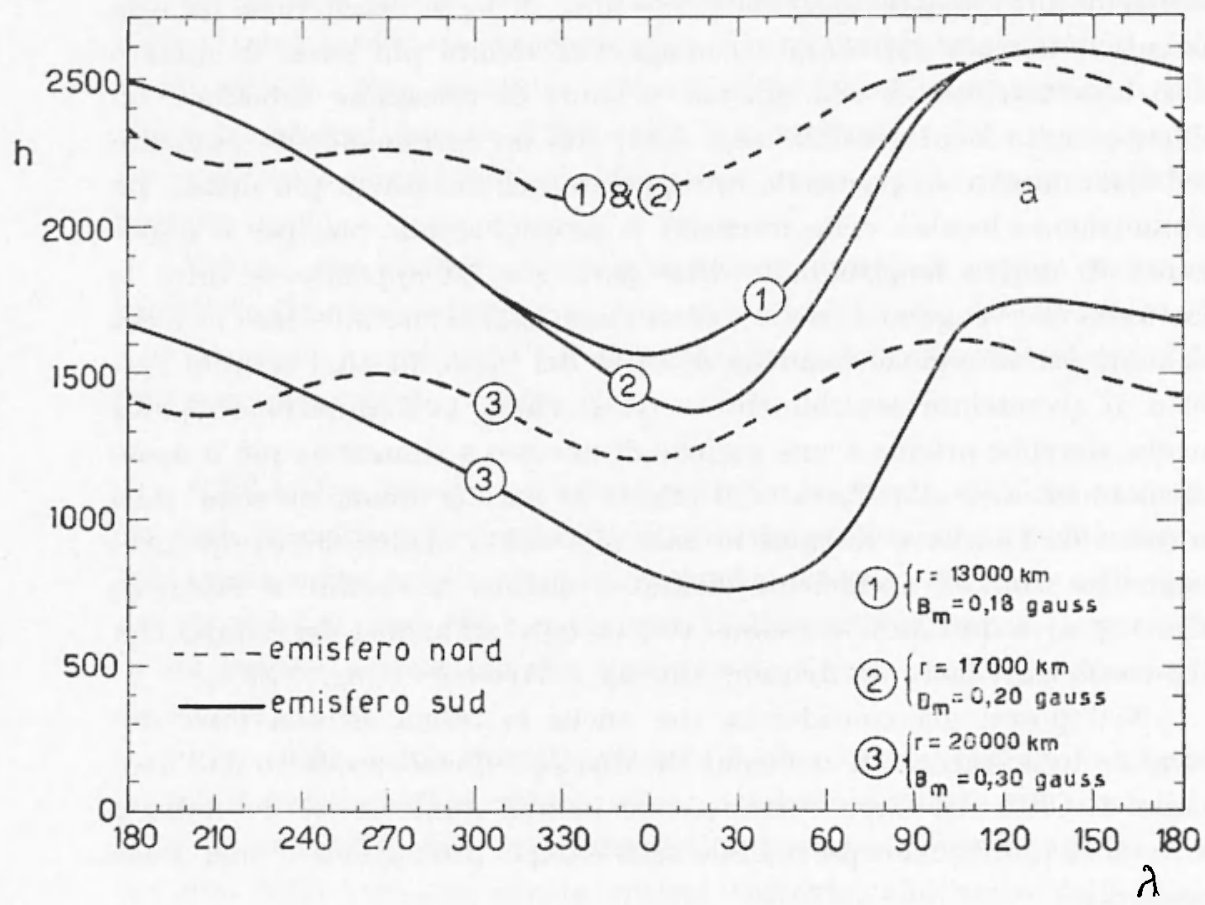

Fig. 5.36 a - Andamento longitudinale dellaltezza $h$ (in $\mathrm{km}$ ) sul suolo delle linee di nguale intensitit $B_{m}$ del campo geomagnetieo. nellemisfero nord e nell'enisfero sud: le tre linee per ciaseun Pmisfero si riferiscono a tre diverse distanze geocentriche $r$ delle lines di forza nel piano equatoriale (secondo Dessler a Karplus);

limiti estremi di circa 12.000 e $16.000 \mathrm{~km}$, mentre la distanza sperimentalmente osservata risulta intorno ai $20.000 \mathrm{~km}$. Ciò deriva sia dai calcoli di Hoflman (133) basati sull'analisi del campo geomagnetico di Jensen, Murray e Welch, sia dal fatto che l'invariante adiabatico $L$ per particelle che hamo punti di riflessione a quote di $1000 \div 2000 \mathrm{~km}$ sulla anomalia di Città del Capo è compreso tra i limiti approssimativi di $2 \div 2,5$ ragoi terrestri. 
Allo stato delle cose è probabile che la esistenza del minino sia essenzialmente dovuta alla forma dello spettro e alle particolarità dei rivelatori (soglie, spessori, efficienze), se, come si è detto in 5.3.3.2, i flussi totali di elettroni di energia $T>40 \mathrm{keV}$ sono praticamente costanti per distanze equatoriali tra $L=2$ ed $L=10$ raggi terrestri. Ugualmente distribuita in modo press'a poeo uniforme sembrerebbe la

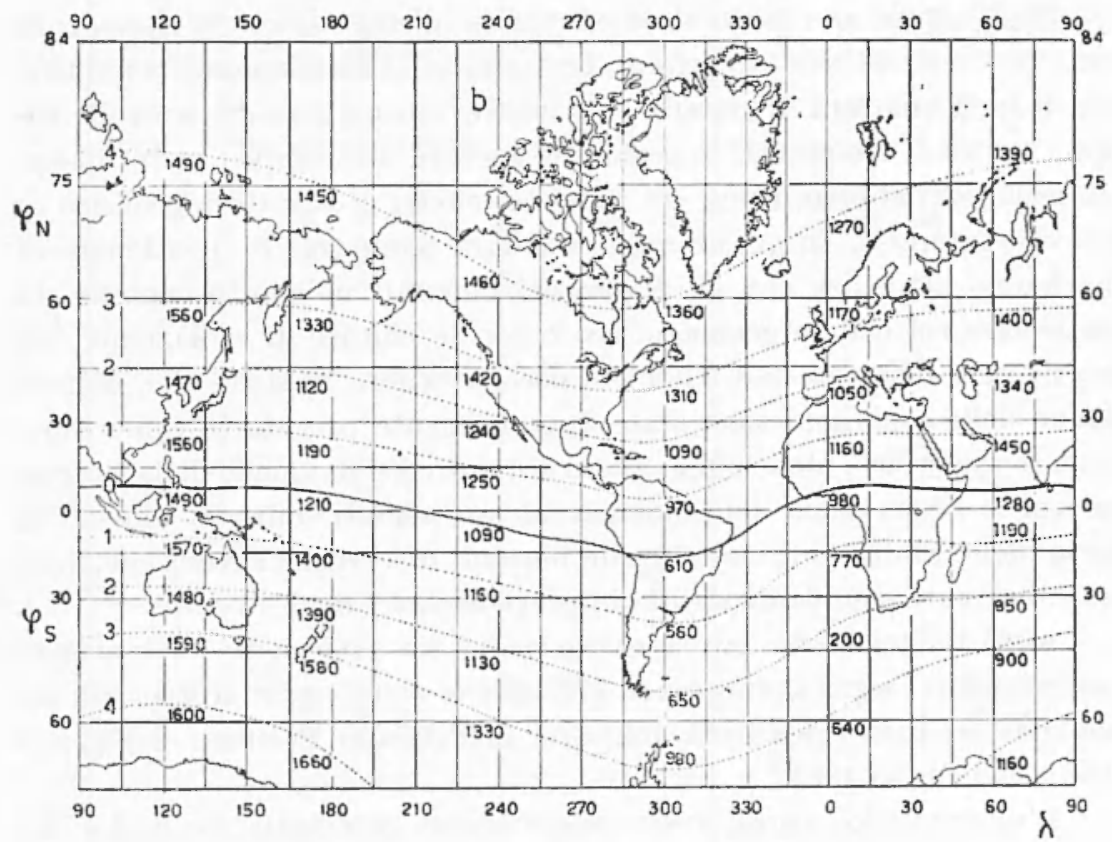

Fig. 5.36 b - Trace sulla superficie terrestre della traietoria sulla quale avviene il moto di deriva delle particelle nei punti di riflessione: si riportano le altezze sul suolo (in $\mathrm{km}$ ) a intervalli di $60^{\circ}$ di longitudine; le curve indicate con le coppie di numeri 1. 2, 3, 4 rappresentano le tracee per punti di riflessione coniugati; la eurva inticala con 0 rappresenta la tratettoria per particelle aventi angolo di inclinazione costante uguale a $90^{\circ}$ (secondo Clardis a Dessler).

sorgente di tali elettroni, proprio conce accarle per es. per le soromenti della emissione elettromagnetica nella esosfera (v. 5.2.2).

Una distribuzione pressoché uniforme del flusso di particelle in tutta la regione di intrappolamento può rendere artificiosa la distinzione di varie zone of fasce, le quali sarebbero solo un effet to indotto dalle par- 
ticolari caratteristiche e dalla particolare lecriones spettrale livelati dai diversi tipi di strumenti.

\subsection{Effetti geofisici delle esplosioni nucleari ad alta quota.}

Segli ultimi amni sono state effettuate alcune esplosioni nucleari di varia potenza nellalta atmosfera. Trattandosi di sprigionamenti improvvisi di forti quantità di energia e di un elevato numero di particelle ariche, e quindi ionizzanti, in legioni di densità atmosferica molto bassa, tali esplosioni ebbero, come era previsto, effetti di carattere geofisico di notevole interesse. In questo numero si farà cemno solo di quei fenomeni che hamo attinenza con gli argomenti illustrati in questo capitolo, in particolare col campo geomagnetico a con le cinture di radiazione. Sel descrivere e discutere tali fenomeni occorre tenere presente che, mentre alcune delle esplosioni sono state appositamente progettate come esperimento scientifico, altre hamno avuto scopi diversi da quello della ricel'a, per cui le osservazioni dei fenomeni ad esse seguiti soffrono, almeno in parte, delle limitazioni inerenti alle normali osservazioni geofisiche, limitazioni dovute soprattutto alla "imprevedibilità » degli eventi.

Appartengono alla prima categoria le tre esplosioni del cosidetto "esperimento Argus", effettuate nell'agosto e settembre 1958, alla seconda le esplosioni dell'Isola Johnston nell'Oceano Pacifico, nell'agosto 1958 a nel luglio 1962.

L'esperimento Argus aveva come scopo principale lo studio del comportamento di particelle ariche aventi energia relativamente moderata (in particolare elettroni di disintegrazione dei prodotti di fissione) nel campo magnetion terrestre: la teoria prevedeva un intrappolamento delle particelle da parte del campo, e siccome al momento della prima ideazione del progetto le fasce di radiazione di van Allen non erano state ancora scoperte (la loro individuazione avvenne durante la fase di elaborazione dell'esperimento Argus) ela di grande interesse, anche in vista di particolari studi sulla fusione termonucleare controllata, verificarne la ralidità. Le tre esplosioni, che eostituivano le iniezioni di elettroni nel campo geomagnetico, vennero effettuate nell'Atlantico meridionale; nella tabella 5.6 vengono riportati i dati più importanti delle esplosioni.

Nella tabella 5.7 riportiamo i dati finora noti sulle esplosioni presso l'Isola Johnston (commicati dalla Atomic Energy Commission). 
Tabulla 5.6.

\begin{tabular}{|c|c|c|c|c|c|c|}
\hline & 1) a 1 a & 'T.M.ti. & $p$ & $\lambda$. & Quot:at & Vinergia \\
\hline $\operatorname{Argus} 12$ & 27 agosto 1958. . . . & 0230 & 38 & $3+s^{\circ} \mathrm{E}$ & $+80 \mathrm{~km}$ & $1-2$ kilotom \\
\hline Arums II : & 30 agosto l95s. . . . & 0320 & 5004 & $352^{\circ} \mathrm{L}$ & $+80 \mathrm{~km}$ & 1-2 kiloton \\
\hline Argus III & fisetiemble loss. . & 2210 & 50 & $3500 \mathrm{E}$ & $480 \mathrm{~km}$ & $1-2$ kiloton \\
\hline
\end{tabular}

Tabollat 5.7

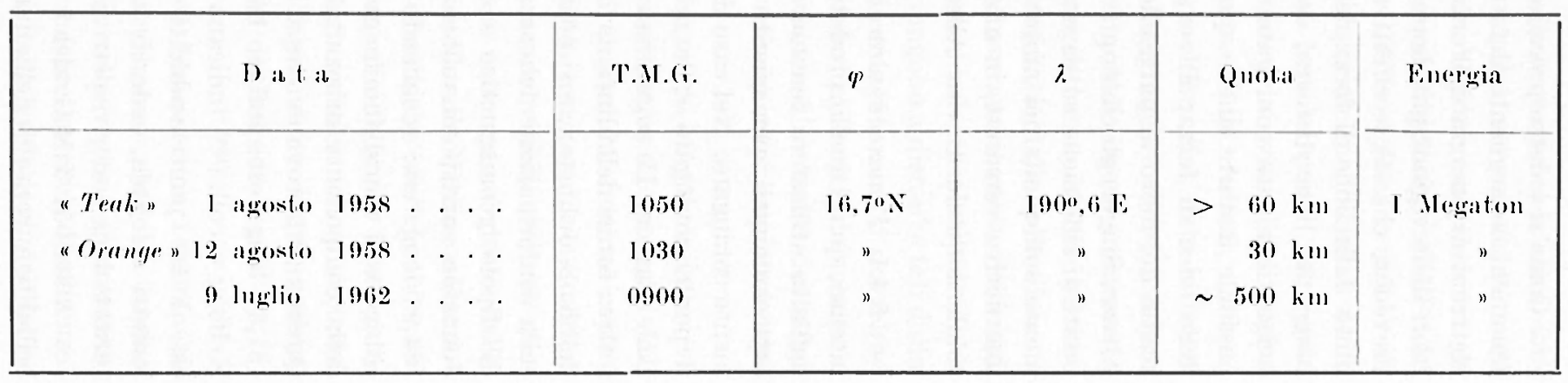


Come si è detto precerlentemente, accenneremo ora solamente a quei fenomeni conseguenti al fatto della iniezione di ma grande quantità di elettroni di energia dell'ordine dei $\mathrm{I}$ (eV nel campo geomagnetico, al di fuori delle regioni più dense dell'atmosfera. In tali condizioni dobbiamo prevedere che: 1) se effettivamente gli elettroni vengono guidati nel loro moto dalle linee di forza del campo manetico terrestre (moto a spilale lungo tali lines di forza) essi derono aadere in gran numero nel punto magneticamente coniugato a quello della esplosione; in tal caso sarà possibile dedure anche qualche informazione sull'andamento efiettivo delle linee di forza; 2) se sono valide le varie teorie elaborate per lo studio del moto di particelle (ariche nel campo geomagnetico (Stormer, Alfven, Singer) gli elettroni devono "rimbalzare " ripetutamente fra i due punti di riflessione su di una linea di forza sui quali ci siamo ripetutamente soffermati nei numeri precerlenti; e contemporaneamente subire una deriva verso est, in modo da formare attorno alla Terra ma fascia simile a quelle di van Allen.

5.4.1. Fenomeni aurorali. - Un fusso di elettroni sufficientemente intenso, quale quello prodotto in una esplosione nucleare, deve produrre nellatta atmosfera fenomeni auromali non solo nei pressi del punto di esplosione, ma, per quanto or ora detto, anche nel punto magneticamente coniugato. Nel caso delle esplosioni al di sopra dell'Isola Johnston il punto coniugato cade nella regione immediatamente and orest delle Isole Samoa. Te coordinate esatte di tale punto dipendono dalla effettiva forma della linea di forza che tocea la superfieie terrestre nei pressi dell'Isola Johnston, e si possono determinare ponendo particolari ipotesi sulla conformazione del ampo magnetico terrestre. Assumendo il campo di dipolo geomagnetico convenzionale, il punto coniugato dell'Isola Johnston corrisponderebbe alle coordinate geografiche 11, 50 lat. sud e $184,9^{\circ}$ long. est; seegliendo invece come riferimento lequatore isorlinico e il meridiano magnetico quale risulta dalla effettiva direzione della componente orizzontale in superficie, Matsushita $\left.{ }^{134}\right)$ ed Elliot a Quenby (355) trovano che il punto coniugato giace a $14,5^{\circ}$ lat. sud e $184,5^{\circ}$ long. est; infine, basandosi sull'equatore dei langgi cosmici, Kellogg e coll. $\left({ }^{136}\right)$ indicano un punto a cirea $17^{\circ}$ lat. sud a $185^{\circ}$ long. est. I tre punti suddetti si trovano tutti a qualche centinaio di chilometri da Apia, secle di un Osservatorio Geofisico (13048' lat. sud, 188014' long. est), rispettivamente nelle direzioni NO, OSO, SO.

Alle 1051 T.ML.G. del 1 agosto 1958 è stata osservata ad dpia una brillante aurom ( $\left.{ }^{137}\right)$ della durata complessiva di 14 minuti; data l'assenza 
di forti tempeste magnetiche, tale fenomeno deve essere senz'altro connesso alla esplosione "Teak" (tab. 5.7). Per i primi sei minuti l'aurora si presentò sotto forma di raggi brillanti in una regione compresa tra gli azimut di circa $235^{\circ}$ a $286^{\circ}$; l'altezza dei raggi raggiungeva i 120. In seguito i raggi diedero luogo ad un chiarore diffuso (glow, r. 2.1.1) di colore rosso, avente il centro approssimatiramente nell'azimut $247^{\circ}$.

La regione aurorale corrisponde piuttosto bene al punto coningato calcolato con riferimento all'equatore isoclinico e ai valori del campo superficiale. Ciò ela abbastanza facilmente prevedibile. Infatti, qualunque sia la forma eflettiva della linea di forza, è chiaro che, data la bassa latitudine dell'Isola Johnston, tale linea può raggiungere come massima altezza i $600 \mathrm{~km}$ dalla superficie; essa deve quindi essere influenzata dalla distribuzione superficiale del campo magnetico, e inoltre rimane troppo vicina alla superficie per essere interessata dalle più distanti regioni del campo magnetico terrestre che influenzano il moto dei raggi cosmici. Secondo Elliot e Quenby, ma esplosione nucleare a latitudini elevate dovebbe interessare linee di forza che giumgono a distanze tali dalla superficie terrestre che per calcolare il punto coniugato non si dovrebbe più fare uso del campo quale si deduce dai valori misurati al snolo, ma del campo esterno quale risulta dalla distribuzione d'intensità dei raggi cosmici, ossia si dorrebbe assumere come riferimento l'equatore dei latgroi cosmici.

Le esplosioni dell'esperimento Argus avvemnero effettivamente (v. tab. 5.6) a latitudini più elevate, specialmente Argus II a Argus III (500 lat. sud). I punti coniugati ai luoghi di queste due ultime esplosioni si trovano presso le Isole Azzorre. Dopo la terza esplosione venne osservata in questa regione una notevole aurora visibile $\left({ }^{138}\right)$ ed una radioaturora $\left({ }^{139}\right)$. Secondo Peterson $\left({ }^{139}\right)$ il punto coniugato dedotto dalle osservazioni dista meno di $100 \mathrm{~km}$ dal punto calcolato da Festine e Karzas dallo sviluppo del campo geomagnetico in armoniche sferiche con 18 coefficienti all'epoca 1955. La fig. 5.2 mostra che il punto coniugato calcolato con riferimento all'equatore dei raggi cosmiei non si accorda bene con le osservazioni. Questo risultato dell'esperimento Argus sarebbe quindi a sfavore della ipotesi de] dipolo rotato di Simpson e coll. $\left.{ }^{2}\right)$.

5.4.2. Effetti geomagnetici e ionosferiei. - Insieme con i fenomeni aurorali, le esplosioni nucleari ad alta quota hanno prodotto anche sensibili effetti geomangnetici e ionosferici. sia nella regione circostante lo scoppio sia nella regione coniugata. E da notare tuttaria l'esistenza di noteroli differenze tra gli eftetti prodotti dalle esplosioni dell'Isola 
Johnston e quelli prodotti dall'esperimento Argus, differenze dovute evidentemente alla diversa altezza alla quale le esplosioni si sono verificate.

Dopo le esplosioni dell'Isola Johnston del 1958 sono state registrate sensibili perturbazioni magnetiche non solo negli Osservatori a distanza relativamente piccola (Honolulu, Isole Palmyla, Fanning e Jarvis), ma anche ad Apia, dove l'ampiezza della perturbazione ha raggriunto il 1 agosto i 120 gamma; è però da osservare che a questa ampiezza contribuisce una perturbazione preesistente del tipo di una baia. Non è faceile stabilire con certezza il processo che ha dato luogo a tali perturbazioni; Matsushita $\left({ }^{134}\right)$ e Mr Nish $\left({ }^{140}\right)$ ne damno due interpretazioni diverse. Ma in questa sede interessa soprattutto il fatto che anche i fenomeni magnetici ad Apia confermano, come l'aulora ivi osservata, l'incamalamento degli elettroni lungo le linee di forza del campo magnetico terrestre.

Durante l'esperinento Argus, invece, non si sono avute perturbazioni del tipo di quelle precedenti, na si è verificata la propagazione su buona parte del globo di onde idromagnetiche, che si sono manifestate nei vari luoghi di osservazione con micropulsazioni del campo magnetieo terrestre o delle correnti telluriche. Selzer (141) hat osservato micropulsazioni evidentemente connesse con l'esperimento Argus in Francial, nelle regioni equatoriali, nell'Isola Kerguelen e nell'Antartide; Berthold e coll. (142) hammo potuto registrare analoghe perturbazioni negli Stati Uniti (New Jersey e Arizona). L'ampiezza di tali oscillazioni variava dai circal 5 gamma nell'Antarticle (Base Dumont d'Trville, lat. geomagn. 75o sud) ai $2 \cdot 10^{-3}$ gamma nell'Arizona. Dall'esame dei tempi di arrivo in vari punti della Terra. Berthold e coll. hamno dedotto dne velocita di propagazione delle onde idromagnetiche, dell:ordine dei 3000 e dei $700 \mathrm{~km} / \mathrm{sec}$ : dallo studio delle ampiezze, inoltre, gli Autori hanno concluso che lattennazione delle onde è una funzione dellat direzione di propagazione, ed è minima lungo il meridiano geomagnetico.

Anche gri effetti ionosferici sembrano confermare, soprattutto nelle esplosioni dell'Isola Johnston, l’inportanza delle linee di forza del campo magnetico nella " trasnissione " di fenomeni elettriei e magnetici. Sensibili perturbazioni ionosferiche sono state infatti registrate a Rarotongar (21,20 lat. sud, 200,20 long. est), a un migliaio di chilometri a sud-est di Apia. Cummack e King (1+3), dallesame degli ionogrammi di Rarotonga, trovano un forte aumento della densità elettronica della regione $F$ da 40 a 50 minuti dopo l'esplosione "Teak ", seguito, nell'ora successiva, da una rapicla diminuzione fino alla scomparsa della regione $F$ normale: in realtà tale fenomeno, più che indicare un effetto guida delle 
linee di forza del campo magnetico, può essere interpretato, come fanno gli Autori, come dovuto a ionizzazione per collisione seguita da scomparsa degli elettroni per attaccamento o ricombinazione; la forte ionizzazione sarebbe a sua volta il prodotto di una specie di onda d'urto propagantesi dal punto della esplosione. Più indicativo di un incanalamento lungo le linee di forza potrebbe essere invece il fatto, segnalato da Uyeda e Ishikawa $\left({ }^{144}\right)$, di un aumento di assorbimento nella regione $D$ a Rarotonga avente inizio cinque ore dopo l'esplosione, massimo dopo sette ore e durato per circa 14 ore. Gli Autori attribuiscono questo fatto agli elettroni emessi dalla nuvola ladioattiva che si spostava lentamente nell'alta atmosfera e guidati dalle linee di forza; quando la nuvola ha cominciato a ricoprire, nel suo moto, la regione coniugata a Rarotonga, si è iniziato in tale regione l'aumento di ionizzazione della regione $D$, con conseguente aumento di assorbimento degli echi riflessi dalle regioni ionosferiche soprastanti.

5.4.3. Particelle intrappolate. - In base alla teoria, gli elettroni iniettati nel campo geomagnetico dalle esplosioni dellesperimento Argus dovevano formare un involucro che, data la latitudine delle esplosioni, era compreso fra la fascia interna e la fascia esterna di van Allen. Questo involucro venme pivelato e studiato da van Allen e coll. $\left.{ }^{145}\right)$ mediante Explorer IV, e da Allen e coll. $\left({ }^{146}\right)$ mediante lazzi lanciati fino ad altezze di $800 \mathrm{~km}$ da località degli Stati Uniti (Project Jason).

I risultati oftenuti dai due gruppi di ricercatori sono, nelle conclusioni fondamentali, assai concordanti fra loro. E stata osservata la caratteristica forma a disco della distribuzione degli elettroni (v. 5.3.2), nel senso che il flusso elettronico era limitato a piani quasi perpenclicolari alle linee di forza, il che è previsto appunto nei pressi dei punti di riflessione. I'involucro presentara un certo spessore, con l'intensità massima delle particelle al centro; lo spessore medio dell'involucro, definito come distanza fra due livelli, simmetrici rispetto al centro, a cui l'intensita e) la metà di quella massima, era, secondo le misure di Explorer IV a varie altezze e latitudini, di $90 \mathrm{~km}$ per Argus I e II e di 150 per Argus III; secondo il gruppo del Project Jason, che ha particolarmente studiato Argus II, lo spessore ela in questo caso, a $800 \mathrm{~km}$ di quota e a $30^{\circ}$ di latitudine, di circa $20 \mathrm{~km}$. Sia le misure di Explorel IV che quelle del Project Jason hanno permesso di concludere che lo spessore dell'involucro e rimasto per tutto il tempo di vita di quest'ultimo praticamente costante; cio significa che le particelle non presentavano una diffusione trasversale alle linee di forza, il che rappresenta una conferma alla esi- 
stenza dell'invilliante $I$ (v. 5.3.2.1). In:alt ra conferma i data dal fatto che anche la posizione dell'involucero rimaneva costante: dalle misure mediante i razzi del Project Jason è risultato che la fascia di elettroni ra esse messa in luce subito dopo Argus II lungo la linea di forza intersecante la superficie terrestre in un punto a 33,50 lat. nord e $285^{\circ}$ long. est non ha mutato di posizione in tutto il periodo di osservazione (t gioprni per un totale di 10 lanci); dal canto loro van Sllen e coll. hamno dedotto la stessa conclusione dallo studio dei punti di intersezione con la superficie terrestre delle linee di forza passanti per il centro dell'involucro, per ognumo dei tre erenti Argus.

Un altro risultato concordante per i due gruppi è quello relativo al deardimento della intensità del fusso delle particelle: l'andamento nel tempo di tale intensitì è della forma $1 / t$, in accordo con l'ipotesi che la perclita delle particelle avvenga per diflusione nella atmosfera nelle vicinanze dei punti di riflessione. Secondo van Allen e coll. l'involucro prodotto da Argus III, nella esplosione del 6 settembre 1958, ela ancola rivelabile quindici giomi dopo, e probabilmente una traceia di esso venne registrata dal Pioneer III il 6 dicembre dello stesso ammo.

Delle conseguenze della esplosione del 9 lughlio 1962 al di sopra della Isola Johnston si ha uno sturlio prelininare di O'Brien e coll. (1+7) i quali hanno utilizzato le misure del satellite Injun I. A differenza delle esplosioni dell'agosto 1958, che, per la bassa quota alle quali sono avvenute, non hanno potuto influenzare la cintura interna di van Allen, lo scoppio del 9 luglio 1962, a qualche rentinaio di chilometri di altezza, ha anmentato notevolmente l'intensità della cintura intema, soprattutto nel ammoo degli elettroni di più elevata energia $(T \geqslant 6$ MeV). Secondo ggli Autori, t5 minuti dopo l'esplosione le intensità regristrate al di sopra de] Sud Africa (per $L=1,32$; altezza intorno a $1000 \mathrm{~km}$ ) in direzione nomale a quella del campo magnetico erano le seguenti:

$$
\begin{aligned}
& \text { per } T \geqslant 40 \mathrm{keV} \quad 3 \cdot 10^{2} \text { particelle } \cdot\left(\mathrm{mi}^{-2} \cdot \mathrm{sec}^{-1} \cdot \mathrm{ster}^{2} \mathrm{ad}^{-1}\right.
\end{aligned}
$$

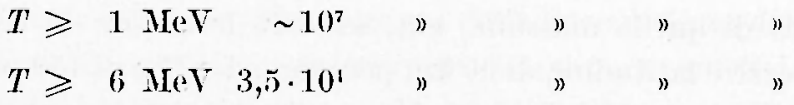

Da un esame dell'andamento della intensitì col tempo gli Autori sono portati a conchudere che, mentre l'intensità degli elettroni $\geqslant 6 \mathrm{MeV}$ aventi punti di riflessione attorno ai $1.10 \mathrm{~km}$ di altezza eara rlivenuta trascumbile in poche ore, l'intensita di quelli riflessi a $350 \mathrm{~km}$ si è ridotta fortemente solo dopo qualche settimana, e infine quella degli elettroni riflessi al di sopra dei $500 \mathrm{~km}$ potrà persistere per alemi mesi. 


\section{BIBJIOGRAFI}

(i) Vestine E. H., Sibley WV. J., Jourh. Geoph. Res. 65, 1967 (1960).

$\left({ }^{2}\right)$ Sinpsox J. A., Fentox K. B., Katzuas J., Rose J). (', Phys, Rev. 102, $1648(1956)$.

$\left({ }^{3}\right)$ IIClOonald F. B., Phys. Rev. 107, 1386 (1957).

(4) Rose I). C., Fentox K. B., Katzuax J., Simpsox J. A., Canad. Journ. Phys., 34, 1 ( $\mathrm{J} 95(\mathrm{i})$.

$\left({ }^{5}\right)$ Rose D. C., l'estox K. B., Katzyax J., Sinpson J. A., Canad. Journ. Phys. 34, 968 (1956).

$\left({ }^{6}\right)$ Daningox R. E., Fremer P. S., Phys. Rev. 109, 151 (I958).

(7) Stoney J. R., Fentox A. G., IICCrackex K. G., Nature 181, 34 (1958).

$\left({ }^{8}\right)$ Poneraxtz M. A., Sindoströn A. E., Rose 1). C., Suppl, Nuovo Cim. $8,257(1958)$.

$\left({ }^{9}\right)$ Katz J., Inyer P., Simpsox J. A., Suppl. Nuovo Gim. 8, 277 (1958).

$\left.{ }^{10}\right)$ Rothwel. P., Quexby .J. J., Suppl. Nuovo Oim. 8, 249 (1958).

(11) Quexis J. J., Wenber WV. R., Phil. Mag. 4, 90 (1959).

(12) Ponerastz M. A., Potnis V. R., Journ. Geoph. Res. 65, 3539 (1960).

(13) Protrzer G., Suppl. Nuovo (im. 8, 220 (1958).

(14) Wixckeder J. R., Axpensos K. A., Phys. Rev, 108, J48 (1957).

$\left({ }^{15}\right)$ Pomerantz M. A., McClune G. W., Phys. Rev. 86, 536 (1952).

${ }^{(16)}$ Roterwels P., Phil. Mag. 3, 961 (1958).

(17) Keldogg P. J., Schwartz M., Nuovo Cim. 13, 761 (1959).

$\left.{ }^{18}\right)$ Quexby J. J., Wesk G. J., Imperial College of science and Tecnolog!!, Jondra, preprint (1961).

$\left({ }^{19}\right)$ Beiser A., Nuovo Cim. 8, 160 (1958).

${ }^{(20)}$ INGiraila R., Nuovo Cim. 12, 356 (1959).

(21) J.UST R., Scmlüter A., Z. Astrophys. 34. 263 (1954).

${ }^{(22)}$ Dolignov S. Sil., Grosinexko E. G., Ziruzgov J. N., Pushkov N. V., 'Tyunmixa J. O., Proc. First Tut. Space Science S'ymp. Nice, 863 (1960).

${ }^{23}$ ) Heppxer J. P., Stolatrik J. D), Simapiro I. R., Cain J. C., Proc. First Int. Space Science Symp. Nice, 982 (1960).

(24) Sonett C. P., Junge D. I., Sins A. R., Kezso J. M., Journ. Geoph. Res. 65, $55(1960)$.

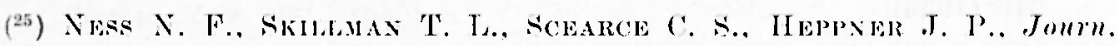
Plyys. Soe. Japan 17, Suppl. A IJ. 27 (1962). 


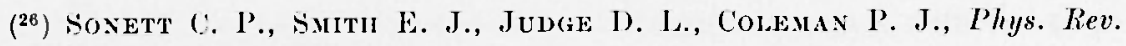
Letters 4, $161(1960)$.

$\left.{ }^{27}\right)$ Sonett (. P., Surti E. .I., Sims A. R., Proc. First Int. Space science symp. Nice, $921(1960)$.

${ }^{28}$ ) SMith E. J., Colenax P. J., Judge I). L., Sonett ('. P., Journ. Geoph. Res. 65, $1858(1960)$.

${ }^{(29)}$ Col.emax P. J., Sonett (. P', Junge D). L., Smitu E. J., Journ. Geoph. Res. 65, $1856(1960)$.

${ }^{(30}$ ) Colemax P'. J. Jr., Invis L., Sonett (. P., Phys. Rev. Letters 5, 43 (1960).

(31) Cotemax P. .I. Jr., Sonett (. P., Davis L., Journ. Geoph. Res. 66, $2043(1961)$.

(32) Gringauz K. I., RYtov S. II., Doklady Akad. Nauk. SSSR 135, 48 (1960), tradotto in Soviet Phys. Dokl. 5, 1225 (196I).

${ }^{(33)}$ Gringauz K. I., Bezrukiki V. V., Ozerov V. D., Rybcinnski R. E., Doklady ikad. Nank. SSSR 131, 1301 (1960), tradotto in Soviet Phys. Dokl. 5, 361 (1960).

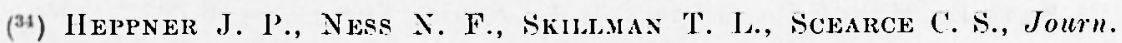
Phys. Soc. Japan 17, Suppl. AIT, 546 (1962).

$\left({ }^{35}\right)$ Sirtu E. J., Sonet' C. P', Journ. Phys. Soc. Japan, 17, Suppl. AJ, 17 (1962).

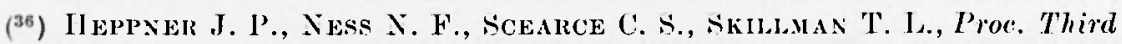
Int. Space Science Symp. Washington, (magrgio 1962).

$\left({ }^{37}\right)$ Fan C. Y., Meyer P., Simpson J. A., Journ. Geoph. Res, 65, 1862 (1960).

( $\left.{ }^{38}\right)$ Barkinusen II., P'lys. Z. 20, 401 (1919).

$\left({ }^{39}\right)$ Stoney L. R. O., Phil. Trans. Roy. Soc. I 246. 113 (1953).

$\left({ }^{40}\right)$ IIeldiweld R. A., Morgax M. G., P'ire 47, 200 (1959).

(41) Morgan M. G., Aldcock G. MI., Nature 177, 30 (1956).

(42) Cain J. C., Silapiro I. R., Stolarik J. D., Ileppner J. P', Journ. Pliys. Soc. Japan, 17, Suppl. AII, 84 (1962).

${ }^{(43)}$ Inh'TIs L. II., Nature 181, $1796(1958)$.

(44) Martin L. II., Nalure 187, 1018 (1960).

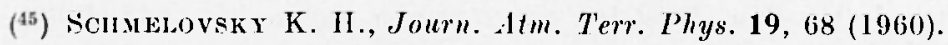

${ }^{(46}$ ) Iwai A., Outsu J., Proe. Res. Inst. Atmosph. Nagoya Univ. 5, 50 (1958).

( $\left.{ }^{47}\right)$ Aldcock G. MI., Morgan M. G., Journ. Geoph. Res. 63, 573 (1958).

${ }^{(48)}$ RyLe II., Solar Eclipses and the Ionosphere, Porganon Press, Londra, 246 (1956).

(19) Chapyax S., Journ. Geoph. Res. 61, 350 (1956). 
(50) Chapmax S., Journ. Atm. Terr. Phys. 15, 43 (1959).

(51) Heldiwell R. A., Journ. Res. Nat. Bureau Standard 64 D, 642 (1960).

(52) Allcock G. M., Austral. Journ. Phys. 10, 285 (1957).

${ }^{(53)}$ Ellis G. R. A., Nature 186, 229 (1960).

$\left({ }^{54}\right)$ Eutis G. R. A., Journ. Geoph. Res. 65, 1705 (1960).

${ }^{(55)}$ Yosilida s., IIntanaka T., Journ. Phys. Soe. Japan 17, Suppl. AII, $78(1962)$.

(56) Gallet R. M., Helliwell R. A., Journ. Res. Nat. Bureau Standards $63 \mathrm{D}, 21(1959)$.

(57) MacArthur J. W., Phys. Rev. Letters 2, 491 (1959).

${ }^{(58)}$ Ellis G. R., Journ. Atm. Terr. Plyys. 10, 302 (1957).

(59) Bauer S. 1., Daniels F. B., Journ. (ieoph. Res. 64, 1371 (1959).

$\left({ }^{\circ 0}\right)$ B Lack well D. E., Nature 181, 1237 (1958).

(61) Van Allen J. A., Joint Meeting of Nation. Acad. of Sci. and Amer. Phys. Soc., 10 maggio 1958.

(62) van Allen J. A., Ludwig G. H., Ray E. C., MeIluwain C. E., Jet Propulsion 28, 588 (1958).

(63) Vernov S. N., Grigorov N. L., Logachev Yu. I., Chumakov A. Ye., Fifth General Assembly CSACI, Mosca 1958, in Annals of $I G Y, \mathrm{XII}$, II, 650 (1961).

(61) Vernov S. N., Vakulov P. V., Gorchakov E. V., Logachev Yu. I. Cnudakov A. Ye., Fifth General Assembly CSAGL, Mosea 1958, in Annals of $I G Y, \mathrm{XII}, \mathrm{II}, 658$ (1961).

${ }^{(65)}$ Vernov S. N., Chudakov A. Ye., Gorchakov li. V., Logachev Yu. I., Vakulov P. V., Plan. Space Science 1, 86 (1959).

${ }^{(66)}$ van Allen J. A., Mcluwain G. E., Ludwig G. II., Journ. Geoph. Res. 64, $271(1959)$.

${ }^{67}$ Rosen A., Sonett C. P., Coleman P. I., Micilwain C. E., Journ. (ieoph. Res. 64, 709 (1959).

${ }^{68}$ ) van Allen J. A., Frank I. A., Nalure 183, 430 (1959).

${ }^{69}$ ) Vernov S. N., Chunakov A. E., Proc. First Int. Space Science Symp. Nice, $751(1960)$.

(70) Vernov s. N., Chudakov A. Ye., Vakulov P. V., Iugachev Yu. I., Nikolaev A. G., Dokladly Alkad. Nauk. 130, 95 (1960), tradotto in Soviet Pliys. 5, 95 (1960).

(i1) van Allen J. A., Frank I. A., Nalure 184, 219 (1959).

(72) Fan C. Y., IIEYer P., Simpson J. A., Proc. First Int. Space Science Symp. Nice, 951 (1960).

${ }^{73}$ ) McIlwain C. E., Journ. Geoph. Res. 66, 3681 (196i). 
(i) Vax Allex J. A., Journ. (ieoph. Res. 64, 1683 (1959).

(i5) O'Briex B. .I., vax Aliex .I. A., Laughlix C. D., Frank L. A., Journ. Geoph. Res. 67, 397 (1962).

(i6) Frenex s. C., White R. S., Phys. Rex. Lelters 3, 9 (1959).

(ii) Frenex s. (. , WhItTE R. S., Journ. Gooph. Res. 65, $137 t$ (1960).

(78) Arustrong A. H., Hakrison F. B., Heckmax H. H., Rosex I., Journ. Geoph. Res. 66, 351 (1961).

(90) Fredex s. (', White R. S., Journ. Geoph. Res. 67, 25 (1962).

(80) Naugie J. F., Kimprex 1). A., Journ. Phys. Soe. Japan 17, Supll. A IJ, $118(1962)$.

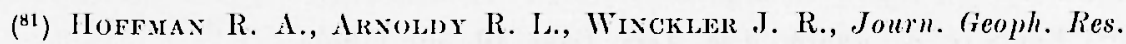
67, I (1962).

${ }^{(82)}$ Holny F. E., Aluex I. J J., Jomnson R. G., Journ. Geoph. Res. 66, $1627(1961)$.

(83) Fremun J. W., Journ. Geoph. Res. 67, 921 (1962).

(81) Holly F. F., Jonssox R. G., Journ. Geoph. Res. 65, 771 (1960).

(85) Davis J. R., Wiblamsox J. M., Proc. Third Int. Space Scienee Symp. Washingron, (magrgio 1962).

(80) Watt M., Cinase L. F., Chadis J. B., Trhof W. I., Knecht D). J., Proc. First Inl. Space Science Symp. Nice, 910 (1960).

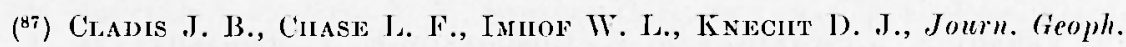
Res. 66, 2297 (1961).

(88) O'BRIEN J., Journ. (ieoph. Res. 67, 3687 (1962).

(89) Dessler A. J., Vestrive E. H., Journ. Geoph. Res. 65, 1069 (1960).

$\left(^{90}\right)$ Pizzelda (4., Mcllwain C. li., van Aldex .J. A., Journ Geoph. Res. 67, $1235(1962)$.

("1) LIx W. C., Slate University Lowa Reporl, 01-16 (1961).

(92) Veriov $s$. N., Chudikov A. Ye., Vakulov P. V., Jogachev Yu. I., Doklady Akad. Nauk. SSSR 125, 304 (1959), tradotto in Sov. Phys. 4, 338 (1959).

(93) Rothwell P., Mcllwain C. li., Journ. Geoph. Res. 65, 799 (1960).

(9.4) Jaccina L., Nralue 183, 1662 (1959).

(25) Arsoldy R., Horinas R., Wixcklen J. R., Journ. Geoph. Res. 65. 1361 (1960).

(116) Axperson K. A., Journ. Geoph. Res. 65, 551 (1960).

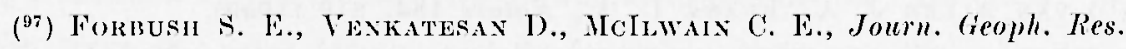
66, $2275(1961)$.

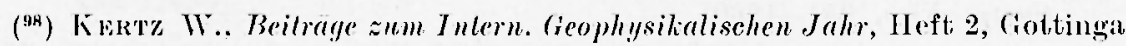
(1958). 
$\left.{ }^{99}\right)$ Rosen A., Fartey 'T. A., Sone't G. P., Proc. First Int. Sipace seience Symp. Nice, 938 (1960).

$\left({ }^{100}\right)$ Rosex A., Fardey T. A., Journ. Geoph. Res. 66, 2013 (1961).

(101) Judge D. L., Colemax P. J., citato in (102).

(102) Farley 'T. A., Rosex A., Journ. Geoph. Res. 65, 349+ (1960).

${ }^{103}$ ) Fax C. I., Meyer P., Simpsox J. A., T'roc. Sec. Int. Space Science Symp. Florence, 867 (1961).

(10:) Stolov II. I., Journ. Geoph. Res. 67, 404 (1962).

$\left(^{105}\right)$ O'Brien B. J., Ijudwig G. II., Journ. Geoph. Res. 65, 2695 (1960).

(106) Keriogra P. J., Nuono Cim. 11, 48 (1959).

$\left.{ }^{107}\right)$ Moxo Y., Kawakami K., Rep. Tonosph. Res. Japan 12, 28 (1958).

(108) Mariaxi F., Journ. (ieoph. Res. 68, in eorso di stampa.

${ }^{(109)}$ Jastrow R., Proc. First Iml. Space Science Symp. Nice, 1009 (1960).

(110) Sixger F., Phys. Rev. Lellers 1, 171 e 181 (1958).

(111) Vernoy S. X., Special Lechre, Fifh General Issembly Cis.lil, Iosca (1958).

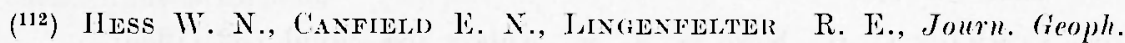
Res. 66, 665 (1961).

(113) Keldogg: P. J., Journ. Geoph. Res. 65, 2705 (1960).

(11.1) Lenchek A. M., Singer S. J', Wextworti R. C., Journ. (ieoph. Res. 66, $4027(1961)$.

(115) Wait M., MacDonald W. M., Joum. Geoph. Res. 66, 2047 (1961).

(116) Dessier A. J., Phys. Lellers 1, 68 (1958).

(117) Fermi E., Phys. Rev. 75, 1169 (1949).

(118) II ess WT. N., Phys. Rev. Tellers 3, 11 (1959).

(119) Iless W. N., Journ. Geoph. Res. 65, 3107 (1960).

$\left.{ }^{120}\right)$ RAY F. C., Jourm. Geoph. Res. 65, 1125 (1960).

(121) Dessier A. J., Katrius R., Phys. Rev. Lellers 4, 271 (1960).

(122) IJess W. N., Krildex J., Journ. Geoph. Res. 66, 3671 (1961).

(123) Iness W. N., Kindeex J., Fax C. T., Meqer P., Srypsox J. A., Journ. Geoph. Res. 66, 2313 (1961).

(12.1) Singer S. F., Thys. Rev. Letlers 3, 188 (1959).

(125) Welci J. A., Wintaker W. A., Joum. Geoph. Res. 64, 909 (1959).

${ }^{(126)}$ Strunte G. W., Phys. Rev. Lellers 2,417 (1959).

(127) Lingoins H., Journ. Geoph. Res. 66. 3593 (1961).

(128) Jastrow R., Rev. Mod. Phys. 32, 947 (1960). 
$\left({ }^{129}\right)$ (ioLI) T., Science 129, 1012 (1959).

(130) Dessler A. .I., Journ. Geoph. Res. 64. 713 (1959).

(131) Cladis J. B., Desslen A. J., Jourm. Geoph. Res. 66, 343 (1961).

(132) Jexsex I). ( .. Murray W., Wedcil J. A., Air Force Spee. Weapoas Center thuquerque Report TN $60-8$ (1960).

(133) Hormuax R. A., Journ. Geoph. Res. 66, 4003 (1961).

(134) Matsusnita S., Journ. Geoph. Res. 64, 1149 (1959).

(135) Eldot II., Quexis J. I., Nature 183, 810 (1959).

(136) Keldoga P. J., Ney E. P., Winckler .I. R., Nature 183, 358 (1959).

(13i) Cullisgtox A. L., Nature 182, 1365 (1958).

(138) Newras P., Journ. (ieoph. Res. 64. 923 (1959).

(139) Petensos A. М., Journ. Gieoph. Res. 64, 933 (1959).

(140) McNisil A. G., Jouru. Geoph. Res. 64, 2253 (1959).

(141) Selzer E., C. R. Acad. Sci. Paris 249, 1133 (1959).

(142) Berthold W. K., Harkis A. K., Hope H. .I., Journ. Geoph. Res. 65. $2233(1960)$.

(143) Cumuack (…,., Kixg G. A. M., Nature 184 Suppl., 32 (1959).

(144) UYEDA H., IsIHKAWA S., Journ. Geomagn. Geoel. 11, 54 (1959).

(145) vax Aldex J. A., McImwax G. E., Lunwig G. H., Journ. Geoph. Res. 64, $877(1959)$.

(146) Allex L., Beavers J. 1., Wintaker W. A., Welch J. A., Walton R. B., Journ. Geoph. Res. 64, 893 (1959).

(147) O'Briex B. .l., InUailis (․ D), vax Alitex J. A., Nalure 195. 939 $(1962)$. 